\title{
Profiling plants to predict range dynamics under climate warming
}

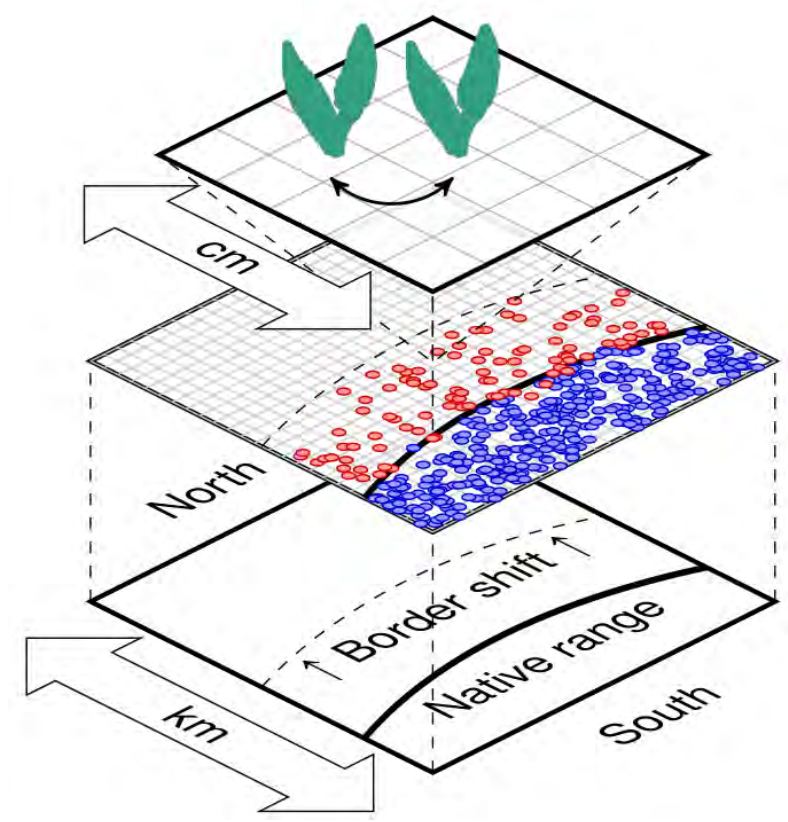

Dissertation

zur Erlangung des Doktorgrades

der Fakultät für Forstwissenschaften und Waldökologie der Georg-August-Universität Göttingen

vorgelegt von

Janina Radny

geboren in

Schönau im Schwarzwald

Göttingen, 2019 
1. Gutachterin: Prof. Dr. Kerstin Wiegand

2. Gutachter: Prof. Dr. Holger Kreft

Tag der mündlichen Prüfung: 22.07.2019 


\section{Contents}

List of Figures $\quad$ iii

List of Tables $\quad$ v

$\begin{array}{ll}\text { Summary } & 1\end{array}$

$\begin{array}{ll}\text { Introduction and scientific context } & 7\end{array}$

1. The fate of plant species under climate warming 13

1.1. Introduction . . . . . . . . . . . . . . . . . . . . . 14

1.2. Projections of climate warming and impacts on plant communities . . . . 15

1.3. Plant responses to climate change . . . . . . . . . . . . . . 20

1.4. Discussion - How to predict species response to climate warming? . . . . . 26

2. Performance of non-native annual plant species in a novel community 29

2.1. Introduction . . . . . . . . . . . . . . . . . . . . . . 30

2.2. Material and methods . . . . . . . . . . . . . . . . . 32

2.3. Results . . . . . . . . . . . . . . . . . . . . . . . 36

2.4. Discussion . . . . . . . . . . . . . . . . . . . . . . . 39

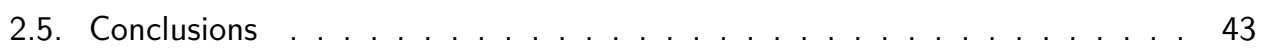

3. Modelling plant establishment in novel communities 45

3.1. Introduction . . . . . . . . . . . . . . . . . . . 46

3.2. Model description . . . . . . . . . . . . . . . . . . . . . . 50

3.3. Results . . . . . . . . . . . . . . . . . . . . . . . . . . 63

3.4. Discussion . . . . . . . . . . . . . . . . . . 67

3.5. Conclusions . . . . . . . . . . . . . . . . 73

4. Macrosystems ecology as conceptual framework to model range dynamics $\mathbf{7 5}$

4.1. Introduction . . . . . . . . . . . . . . . 76

4.2. Hierarchy theory and macrosystems ecology . . . . . . . . . . . . 81

4.3. Climate warming-induced range dynamics in an macrosystems ecology framework . . . . . . . . . . . . . . . . . . . . . 84

4.4. Discussion . . . . . . . . . . . . . . . . . . . . . . . 100

$\begin{array}{ll}\text { 5. Concluding discussion } & 105\end{array}$

$\begin{array}{ll}\text { Literature } & 111\end{array}$

A. Supplemental Material: Alternative herbivory simulations 141

$\begin{array}{ll}\text { Acknowledgements } & 147\end{array}$

$\begin{array}{ll}\text { Declaration } & 151\end{array}$ 



\section{List of Figures}

1.1. The major components of the global climate system . . . . . . . . . . . . 16

1.2. Temporal scales of expected climate warming impacts . . . . . . . . . . . . 17

1.3. Three determinants of habitat suitability . . . . . . . . . . . . . . . . . 19

2.1. Pots with Crepis sancta as non-native species . . . . . . . . . . . . . . . 34

2.2. Dry shoot biomass of non-native species . . . . . . . . . . . . . . . . 38

2.3. Length of longest shoot of non-native plant species. . . . . . . . . . . . . . . . . . . . . . . . . . . . .

2.4. Number of seeds of non-native plant species. . . . . . . . . . . . . . . . 39

2.5. Illustration of dominance of large non-natives . . . . . . . . . . . . . 43

3.1. Schematic overview of relationship between range shift and local establishment 48

3.2. Model flow . . . . . . . . . . . . . . . . . . . . . . 52

3.3. Illustration of the Zone-of-Influence $($ ZOI) . . . . . . . . . . . . . . . 56

3.4. Screenshot of model communities after 14 days . . . . . . . . . . . . . . . 60

3.5. Survival rates of non-native plants with different trait profiles . . . . . . . . 64

3.6. Survival rates of non-native plants under different invasion levels . . . . . . 65

3.7. Effect of the intensity of competition $\Theta$ on survival rates of non-native plants 66

3.8. Survival rate of non-native species at different herbivore densities . . . . . . 67

3.9. Standardized sensitivities of model output to model parameters . . . . . . . 68

3.10. The relationship between plant growth and plant biomass in the model . . 72

4.1. Spatial level, pattern emergence and major processes for the integrated model 86

4.2. Patterns of range dynamics . . . . . . . . . . . . . . . . . . . . . . . . . . . . . . . . . .

4.3. Köppen-Geiger climate type map of Europe . . . . . . . . . . . . . . . . . 96

5.1. Schematic profile examples . . . . . . . . . . . . . . . . 106

A.1. Alternative run: Survival rates of non-native plants with different trait profiles143

A.2. Alternative run: Survival rates of non-native plants under different invasion levels in high density . . . . . . . . . . . . . . . . . . . . . . 143

A.3. Alternative run: Effect of the intensity of competition $\Theta$ on survival rates of non-native plants . . . . . . . . . . . . . . . . . . . . . 144

A.4. Alternative run: Survival rate of non-native species at different herbivore

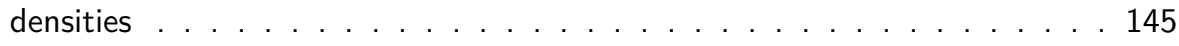





\section{List of Tables}

2.1. List of non-native species used in the experiment . . . . . . . . . . . 33

2.2. Effects of initial seed size, family, and competition treatment . . . . . . . 37

2.3. Relative competition effects on the response variables. . . . . . . . . . . 37

3.1. Parameter values for traits of non-native plants . . . . . . . . . . . . 50

3.2. Parameter values for traits of non-native plants . . . . . . . . . . . . . . 51

3.3. Overview of components of model scenarios . . . . . . . . . . . . . . . . 61

3.4. Effects of traits and community configuration on survival of non-native species 63

4.1. Description of the relevant scales, ecological units and the respective basic entities and units. . . . . . . . . . . . . . . . . . . . 89

5.1. Possible trait combinations . . . . . . . . . . . . . . 106 


\section{Summary}

Global climate warming affects species, ecosystems and entire biomes around the world. Shifts of range borders and distribution patterns along with changing climatic conditions were observed for many plant species. Successful range shift is realized by dispersal to, and establishment in novel habitats. Different abilities to reach and colonize novel habitats results in different range shift patterns and velocities among species.

The overall aim of this thesis was to investigate mechanisms of plant range dynamics under climate warming with a focus on local establishment of novel species in a resident community. Specifically, this thesis explores (1) how ecological profile, expressed as a combination of traits related to competition and defence, affects establishment success of novel plant species in a resident community and (2) how local population dynamics and mesoscale dispersal processes can be integrated to fully describe species range dynamics under climate warming on a macroscale.

To address objective (1), I combined a greenhouse experiment and a spatially explicit traitand individual-based simulation model to assess local plant population dynamics. In the greenhouse experiment, I investigated the effect of biotic pressures, namely herbivory and competition, on the establishment success of eight species from the Mediterranean in a plant community of Central Europe. The experiment assessed how well seed size as a proxy trait of competitive strength explains the response of species to high density of the native community as proxy of competitive pressure. The experiment was conducted with and without herbivore presence. The simulation model was developed based on the greenhouse experiment and added defensive traits to the plant trait profiles. The model assessed population response to initial densities of the resident community and the novel species, composition of the native community, competition intensity and herbivore pressure.

The greenhouse experiment confirmed the potentially large impact of competitive pressure 


\section{Summary}

from resident plants on the performance of novel intruders. However, the relative strength of the negative response was greater for those species labelled as strong competitors than for those labelled as weak competitors. Despite visible herbivore damage on many plants, herbivory did not have a significant impact on plant performance. These two results underline the importance of tolerance to biotic pressures for early establishment in a novel community.

The local individual-based model used the Zone-Of-Influence approach to model competition between plants. Model analysis showed that competitive ability was strongly correlated with individual survival, albeit too many individuals of strong competitors resulted in a selfthinning effect. Herbivory did not markedly affect population growth, but had a slight positive indirect effect on survival of weak competitors. As compared to the experiment, drop-out rate was overestimated in the model. This may be compensated by implementing tolerance mechanisms in future model versions. Furthermore, the degree of competitive asymmetry had a significant effect on plant performance. This calls for including inter- and intraspecific variability in the degree of competitive asymmetry in future model versions to improve model realism by introducing niche differentiation. A sensitivity analysis of the model revealed the importance of interactions of traits with the form of biotic pressures.

With respect to objective (2), I developed a model concept based on macrosystems ecology for scaling up local population dynamics to regional species range dynamics. This concept considers processes on a macro-, meso- and microscale and identifies major crossscale interactions and cross-scale emergence of patterns. The origin of this concept is the observation that the sum of local colonialization and extinction events forms the overall range dynamics pattern on a broader spatial scale. As part of this concept more potential interaction types should be addressed on the local scale than I assessed in the greenhouse experiment or in the model. By assessing a broader picture of possible community interactions as well as interactions across scales in an implementation of this concept, I expect to gain more realistic insights into the processes underlying range dynamics in the future.

Overall, I conclude that range dynamics is more than just the sum of its parts and usually emerges from a highly complex interaction web. This thesis revealed the importance of interactions of species traits and biotic pressures for early establishment of novel plant species. For realistic assessments of future range dynamics of plant species, I join the claim 
to exploit the wealth of the continuously growing data repositories by implementing novel techniques of data analysis and modelling across disciplines.

\section{Zusammenfassung}

Die globale Klimaerwärmung betrifft Arten, ökosysteme und ganze Biome weltweit. Für viele Pflanzenarten wurden Verschiebungen der Grenzen von Verbreitungsgebieten und Verbreitungsmustern beobachtet, die den sich ändernden klimatischen Bedingungen entsprechen. Eine erfolgreiche Verschiebung der Verbreitungsgebiete wird durch die Ausbreitung in neue Lebensräume und die Etablierung in diesen neuen Lebensräumen erreicht. Unterschiedliche Kapazitäten neue Lebensräume zu erreichen und zu kolonisieren führen zu unterschiedlichen Mustern und Geschwindigkeiten der Verschiebung von Verbreitungsgebieten der einzelnen Arten.

Das übergeordnete Ziel dieser Arbeit war es, Mechanismen der durch Klimaerwärmung verursachten änderungen von Verbreitungsgebieten von Pflanzen zu untersuchen. Die lokale Etablierung neuer Arten in einer ansässigen Gemeinschaft war hierbei ein besonderer Schwerpunkt. Insbesondere wird in dieser Arbeit untersucht, (1) wie das ökologische Profil als eine Kombination von Konkurrenz- und Verteidigungs-Merkmalen die erfolgreiche Etablierung neuer Pflanzenarten in einer ansässigen Gemeinschaft beeinflusst und (2) wie die lokale Populationsdynamik mit Ausbreitungsprozessen auf der Mesoskala verbunden werden kann, um die klimaerwärmungsgetriebene Verbreitungsdynamik von Pflanzenarten auf der Makroskala zu beschreiben.

Um Ziel (1) zu erreichen, habe ich einen Gewächshausexperiment und ein räumlich explizites merkmals- und individuenbasiertes Simulationsmodell kombiniert, um die Dynamik der lokalen Pflanzenpopulation zu untersuchen. In diesem Gewächshausexperiment habe ich untersucht, welchen Einfluss die biologischen Interaktionen Herbivorie und Konkurrenz auf den Etablierungserfolg von acht Arten aus dem Mittelmeerraum in einer mitteleuropäischen Pflanzengemeinschaft haben. Das Experiment untersuchte, wie das Merkmal Samengröße als Indikator für Konkurrenzstärke den Einfluss erhöhter Pflanzdichte der einheimischen Gemeinschaft auf neue Arten erklärt. Dabei war die Pflanzdichte ein Indikator für den Konkurrenzdruck, der mit und ohne Herbivorenpräsenz untersucht wurde. Das Simulation- 
smodell wurde auf Grundlage des Gewächshausversuchs entwickelt und erweitert das Merkmalsprofil um Merkmale, welche die Stärke von Verteidigungsmechanismen beschreiben. Das Modell simulierte Populationsdynamiken in Abhängigkeit von unterschiedlichen Anfangsdichten der ansässigen Gemeinschaft sowie der jeweils neu eingeführten Art, von der Zusammensetzung der ansässigen Gemeinschaft, von der Konkurrenzintensität und von dem Herbivorendruck.

Der Gewächshausversuch bestätigte die potenziell großen Auswirkungen des Konkurrenzdrucks durch ansässige Pflanzen auf neue Arten. Neue Arten, die laut Merkmalsprofil als konkurrenzstark galten, wurden stärker negativ von hohem Konkurrenzdruck beeinflusst als Arten mit konkurrenzschwachem Merkmalsprofil. Trotz sichtbarer Herbivorieschäden an vielen Pflanzen hatte Herbivorie keinen signifikanten Einfluss auf die Wachstumsleistung der Pflanzen. Diese beiden Ergebnisse unterstreichen die Bedeutung der Toleranz gegenüber biotischem Druck für die frühe Etablierung einer neuen Art in einer ansässigen Gemeinschaft.

Das lokale Individuenbasierte Modell verwendete den Zone-of-Influence-Ansatz, um die Konkurrenz zwischen Pflanzen durch überlappende Einflussbereiche zu modellieren. Die Modellanalyse zeigte, dass die Konkurrenzfähigkeit stark mit dem individuellen überleben korreliert war, obwohl zu hohe Individuenzahlen starker Konkurrenten zu einem Selbstausdünnungseffekt führten. Herbivorie hatte keinen nennenswerten Einfluss auf das Populationswachstum, hatte aber einen leicht positiven indirekten Einfluss auf das überleben schwacher Konkurrenten. Im Modell starben mehr Pflanzenindividuen als im Experiment. Dies könnte durch die Implementierung von Toleranzmechanismen in zukünftigen Modellversionen kompensiert werden. Darüber hinaus hatte der Grad der Konkurrenzasymmetrie einen signifikanten Einfluss auf die überlebensrate. Diese Erkenntnis unterstreicht die Notwendigkeit, die inter- und intraspezifische Variabilität der Konkurrenzasymmetrie in zukünftige Modellversionen einzubeziehen, um die Realitätsnähe des Modells durch die Einführung von Nischendifferenzierung zu verbessern. Eine Sensitivitätsanalyse des Modells unterstrich die Bedeutung von Wechselwirkungen zwischen Pflanzenmerkmalen und der Form negativer biotischer Interaktionen.

Im Hinblick auf Ziel (2) habe ich ein Modellkonzept entwickelt, das das bestehende "macrosystems ecology"-Konzept nutzt, um lokale Populationsdynamiken auf regionale 
Artverbreitungsdynamiken hoch zu skalieren. Dieses Konzept berücksichtigt Prozesse auf Makro-, Meso- und Mikroebene und identifiziert skalenübergreifende Wechselwirkungen und die skalenübergreifende Entstehung von Mustern. Der Ursprung dieses Konzeptes ist die Beobachtung, dass die Summe von lokalen Besiedelungs- und Aussterbeereignissen die Gesamtdynamik von Artverbreitungsgebieten auf höheren räumlichen Skalen hervorbringt. Als Teil dieses Konzepts sollten mehr potenzielle Interaktionstypen auf lokaler Ebene berücksichtigt werden als ich im Gewächshausexperiment und im Model untersucht habe. Durch die umfassende Einbeziehung möglicher lokaler Interaktionen in einer Gemeinschaft sowie skalenübergreifender Interaktionen wird eine Implementierung dieses Konzepts realistischere Einblicke in die Prozesse bieten, die derzeitigen und zukünftigen Artverbreitungsdynamiken zugrunde liegen.

Insgesamt komme ich zu dem Schluss, dass die Artverbreitungsdynamik mehr ist als nur die Summe ihrer Teile und meist aus einem hochkomplexen Interaktionsnetz hervorgeht. Diese Arbeit offenbart die Bedeutung der Wechselwirkungen von Artmerkmalen und biotischen Interaktionen für die frühe Etablierung neuer Pflanzenarten. Für realistische Einschätzungen der zukünftigen Verbreitungsdynamik von Pflanzenarten schließe ich mich der Forderung an, den ständig wachsenden Datenreichtum durch Anwendung neuer Techniken der Datenanalyse und Modellierung weiter auszuschöpfen. 



\section{Introduction and scientific context}

Global climate warming is altering bioclimatic conditions on a global, regional, and local scale (IPCC, 2014; Williams et al., 2007). Severe negative impacts on biodiversity and ecosystem stability are expected worldwide (Bellard et al., 2012; Jump and Penuelas, 2005; Thuiller et al., 2005; Urban, 2015). Plants are locally affected not only by the rise of mean temperatures, but also by associated effects such as changes in precipitation patterns, shift of seasonality or extreme weather events and these changes can alter local habitat suitability and interaction balances within a community (see chapter 1 ).

A global trend of shifting ranges has been observed during the last decades with a prevailing direction towards higher latitudes and altitudes (Parmesan and Yohe, 2003; Root et al., 2003; Thomas, 2010). Recently, exceptions from that directional trend were more strongly appreciated. In fact, most studies on observed range shifts were reported with outliers of species not moving at all or even in opposing directions (Lenoir and Svenning, 2015; Parmesan and Hanley, 2015). Even for species from the same species pool, movement is not necessarily uniform (Chen et al., 2011; le Roux and McGeoch, 2008; Zhu et al., 2012). Differences in direction as well as speed of range shift may lead to community reshuffling, changing interaction patterns (Alexander et al., 2016; Ma et al., 2017), or spatial segregation of taxa from the same original community (Fei et al., 2017). To give a reasonable prediction of species movement and subsequent reshuffling of local communities, it is important to understand the drivers and processes underlying range shift dynamics.

Diverging movement patterns emerge from different combinations of species' ability to reach a novel habitat, local survival and subsequent establishment success. Species' functional traits can be a mediator for local community assembly, determining overall habitat suitability (Belluau and Shipley, 2018) and affecting biotic interactions or vice versa (Gross et al., 2009). The inherent generality of trait-based approaches makes them highly suit- 
able for an application to range shifts in heterogeneous landscapes and changing bioclimatic conditions (McGill et al., 2006; Violle et al., 2007). Additionally, using traits instead of taxonomic identity to assess interactions could markedly facilitate the analysis of multispecies systems (Berg et al., 2010). This generality may become increasingly important under the assumption of arising no-analogue communities through climate warming-induced range shifts (Williams and Jackson, 2007) and allows for climate warming-related extinction risk assessment beyond taxonomic limitations (Butt and Gallagher, 2018; Estrada et al., 2016; Zakharova et al., 2019).

Range shift of species can be differentiated into stages of movement to, and establishment in novel habitats. For each stage, specific trait sets may be relevant (Estrada et al., 2016). Strong dispersal capacity can help species to react novel habitats faster and thus allow for keeping pace with rapid climate change, but simultaneously increase the possibility to arrive in locations with very different environmental conditions, especially in regions with steep environmental gradients (Boeye et al., 2013; Hargreaves et al., 2015; Phillips, 2012). Relevant dispersal traits are for example the shape of the dispersal kernel (Urban et al., 2013), the capacity of long-distance dispersal (Soons and Ozinga, 2005), or the reliance on dispersal vectors, which can determine prevailing direction and distance for realized dispersal of passively dispersing organisms such as plants (Cunze et al., 2013; Fei et al., 2017). Once propagules of a species have reached a novel habitat, biotic interactions may promote or inhibit colonialization and subsequent establishment in the novel habitat (Estrada et al., 2018; Levine et al., 2004; Morriën et al., 2010; Svenning et al., 2014; Van Der Putten et al., 2010). Factors that inhibit establishment contribute to the biotic resistance of a local community, most prominently the exclusion by superior competitors or herbivory (Erneberg, 1999; le Roux et al., 2012). Assuming that traits mediate competitive interactions (Goldberg and Landa, 1991; Howard and Goldberg, 2001; Kunstler et al., 2016), some species should be less affected by competitive exclusion than others. For example, great biomass is associated with the ability to pre-empt available resources (Schwinning and Weiner, 1998) and may decrease effectiveness of biotic resistance if the novel species is equipped with traits such as larger biomass or seed size (Radny et al., 2018). This effect might be enhanced further when the novel species experiences less herbivory than the resident community (Bossdorf, 2013), for example when the host plant can disperse faster than their specialist enemies. Enemy release is a common motive in intercontinental 
range expansions (Chun et al., 2010) and has also recently been recognized for climate warming-induced range shifts (Engelkes et al., 2008; Lakeman-Fraser and Ewers, 2013; Morriën et al., 2010).

Recently, trait-based approaches become increasingly appreciated in modelling approaches (Zakharova et al., 2019). This may be also due to the ongoing development of modelling techniques. In the last decades, species distribution models have evolved from purely correlative models to more complex mechanistic species distribution models (Singer et al., 2016). These models consider more detailed biotic interactions as fundamental processes of spatiotemporal range dynamics and can outperform purely correlative models that imply interactions only implicitly, if at all. For example, Cabral and Kreft (2012) were able to reproduce realistic distribution patterns for generic species by considering demography, biotic interactions and individual metabolism in their model. The divergence between potential distribution (fundamental niche) and actual distribution (realized niche) highlights the impact of biotic interactions on community assembly and subsequent species sorting. Hence, explicitly considering biotic interactions by including individual-based models into hybrid models may also increase the realism of macroscale predictions of range dynamics (Levy et al., 2014). Additionally, considering biotic interactions allows for more direct parametrization of species distribution models from experimental data (van der Putten et al., 2009).

The overall aim of this thesis was to investigate mechanisms of plant range dynamics under climate warming with a focus on local establishment of novel species in a resident community. Specifically, this thesis explores (1) how ecological profile, expressed as a combination of traits related to competition and defence, affects establishment success of novel plant species in a resident community and (2) how local population dynamics and dispersal processes on a mesoscale can be integrated in a context to fully describe species range dynamics under climate warming on a macroscale. 


\section{Thesis rationale and structure}

This thesis aims to provide insights on climate warming-induced range dynamics of plant species. To this end, I assess early establishment success of novel species in a resident community based on traits that express their competitive and defensive ability. I provide a framework for subsequent modelling of range dynamics on a macroscale. The chapters reflect the consecutive parts of the thesis.

In chapter 1, I provide a brief overview on the mechanisms underlying global climate warming and how plant species are affected by short-term and long-term effects of climate warming. I describe current insights into response strategies of plants, i.e. in situ adaptation and spatial escape strategies. The chapter closes with a brief summary of trait-based approaches that predict the responses of plant species to climate warming. This chapter is a synthesis of the literature I screened during the course of this thesis on topics of global climate warming and its ecological consequences. While the main focus of the thesis is the climate warming-induced range dynamics of plant species, this chapter sets the broader ecological scope for the thesis.

Chapter 2 describes the results of the multispecies greenhouse experiment, conducted at the Netherlands Institute of Ecology NIOO-KNAW in Wageningen, The Netherlands. In cooperation with my co-authors (see full authors list and authors contributions on p. 29), I conducted this experiment to test the hypothesis that the response of plants to the biotic pressures competition and herbivory is mediated by species' traits. This study has been published as Radny et al. (2018) in the journal Acta Oecologica. The contents of this chapter including the abstract is identical with the publication, additional figures are listed on p. 29. Experimental data were used as parameter basis for subsequent modelling.

In chapter 3, I describe the local-scale community model, where I simulated initial establishment of novel species in a resident community, and present and discuss the results of the model runs. I developed the model in close collaboration with my co-author Katrin Meyer (see authors list and declaration of contributions on p. 45). This chapter was published as pre-print and subsequently as peer-reviewed article in the journal PeerJ as Radny and Meyer (2018). The contents of this chapter is identical with the publication.

Appendix $A$ comprises an alternative modelling scenario for herbivory in the model, which 
was published as supplementary material for Radny and Meyer (2018). Authors list and authors contribution are included on p. 45.

In chapter 4, I propose a conceptual framework for modelling species range dynamics on a continental scale. The framework is embedded in the macrosystems ecology framework as proposed by Heffernan et al. (2014). I give a brief introduction into the concept of marcosystems ecology and describe a model framework operating on three spatial scales and organizational levels. The chapter closes with reflections on the implementation of the framework. This work has not been published before and has not yet been submitted to a scientific journal.

Finally, in chapter $\mathbf{5}$ I briefly discuss the results of the thesis project. 



\title{
1. Every step you take: The fate of plant species under climate warming
}

This chapter has not been part of a peer-reviewed publication to date (August 26, 2019), but is left open for publication in the future. JR established the concept and wrote the text. KM improved the manuscript with valuable comments.

Referenced literature is included in the full thesis literature.

\begin{abstract}
Recent global warming threatens biodiversity worldwide. For pro-active conservation management it is necessary to understand how climate warming affects plant species and how they can respond to changing conditions. Here, I summarize how climate warming affects plant species and briefly describe mitigation strategies of species. Accelerated climate warming is the result of an imbalance in the climate system and can be attributed in large parts to human activities. Plants are immediately affected by short-termed extreme weather events, e.g. heavy storms or drought. Long-term change of local climatic conditions can alter local habitat suitability for a species and lead to changes in community stability and composition. Plants can either adapt in situ to changing conditions, resulting in niche shifts or attempt to escape, i.e. shifting their range along with climatic conditions. Where plants fail to adapt or escape, extinction is likely. Which type of response a plant species will expose is highly context-sensitive, e.g. depending on type of environmental stress, community response and overall vulnerability of the focal species. Trait-based approaches can be a key to predict species responses. Explanatory traits should be chosen carefully and different trait sets are likely to be relevant in different stages of environmental changes and response strategies.
\end{abstract}




\subsection{Introduction}

Current global climate warming is a challenge for many organisms worldwide (Loarie et al., 2009; Parmesan and Yohe, 2003; Root et al., 2003; Walther et al., 2005). Global loss of biodiversity is alarming (Bellard et al., 2012; Thomas et al., 2004; Urban, 2015) and decreases the stability and resilience of ecosystems against climate extremes (Isbell et al., 2015; Oliver et al., 2015). Maintenance of crucial ecosystem services and stability is a major societal concern. Hence, for conservation managers as well as policy makers, it is important to know how species will respond to global warming scenarios. Drastically speaking, species' options to face climate warming are: adapt, avoid, or disappear (Bellard et al., 2012; Berg et al., 2010). Here, I will give an overview on impacts of climate warming on plants and the mechanisms underlying their response strategies.

The peculiarity of current climate warming of the post-industrial age is its sheer speed, which is estimated to exceed any other warming phase during the last 66 million years (Diffenbaugh and Field, 2013; IPCC, 2014). Even with current recorded warming of about $1^{\circ} \mathrm{C}$ as compared to pre-industrial times, climate warming triggers changes on levels from genetics and individual physiology to ecosystem productivity and species abundance in terrestrial as well as aquatic ecosystems (Scheffers et al., 2016). A political target of below $2^{\circ} \mathrm{C}$ of global warming was agreed in the Paris agreement (UN, 2015), but pursuing this goal will necessitate resolute actions (Steffen et al., 2018). Under current socio-political developments, the achievability of this target is put in question (Urban, 2015). Additionally, climate warming interacts with further anthropogenic drivers, such as landscape fragmentation, habitat degradation, nutrient eutrophication and continual emission of $\mathrm{CO}_{2}(\mathrm{Hautier}$ et al., 2015; Pedrono et al., 2016; Thompson et al., 2017). Altogether, environmental stress might exceed adaptation potential of species to changing conditions and result in species disappearing on a local scale or even going globally extinct (Cahill et al., 2013).

In the following, I first give a brief introduction into the aspects of global warming that are relevant for plant species. I then review some current insights on in situ adaptation to altered conditions and on range shifts of plant species. To conclude, I discuss how trait-based approaches can be used to assess species' response to climate warming. 


\subsection{Projections of climate warming and impacts on plant communities}

\subsubsection{Climate warming and imbalances of the climate system}

Current climate warming describes the rise of average global temperatures in the industrial and post-industrial age, i.e. since 1850 (IPCC, 2014). In fact, rising temperatures are only the symptom of global climate change, emerging from an imbalance of the global climate system (fig. 1.1). Human activities are very likely to contribute in large parts to this imbalance at subsequent current global warming (Bindoff et al., 2013). This is mostly the emission of greenhouse gases (IPCC, 2014), but other activities aggravate the current development. For example, rapid urbanization creates heat islands and impervious surfaces (Shi et al., 2015); industrial agriculture (Lin et al., 2011) and deforestation (Davin and de Noblet-Ducoudré, 2010) result in large-scale transformation of the biosphere. Besides greenhouse gas emission from burning fossil fuels and stock farming, eutrophication of lakes and rivers creates sources of greenhouse gas, i.e. methane (Sepulveda-Jauregui et al., 2018). Due to the significant impact of humans on the climate system, it has been proposed to include the "anthroposphere" as additional component of the climate system (Gettelman and Rood, 2016).

All components of the climate system do not only influence the system, but in turn are influenced by the climate system as well. For example, rising temperatures cause the polar ice caps to melt, resulting in an altered albedo and further increased warming (Deser et al., 2000). Some parts of the world are expected to experience stronger changes than others (Urban, 2015; Williams and Jackson, 2007), and landscape structure, e.g.mountain ranges, can create further regional heterogeneity (Hannah et al., 2014; Loarie et al., 2009).

Climate warming effects manifest as locally and spatially restricted events, e.g. extreme weather, but also as events and processes that affect several continents through many years, e.g. alteration of large oscillation patterns (Yeh et al., 2009, see also fig. 1.2). Correspondingly, effects can be measured from the level of individual organisms up to the level of entire biomes (Scheffers et al., 2016). 


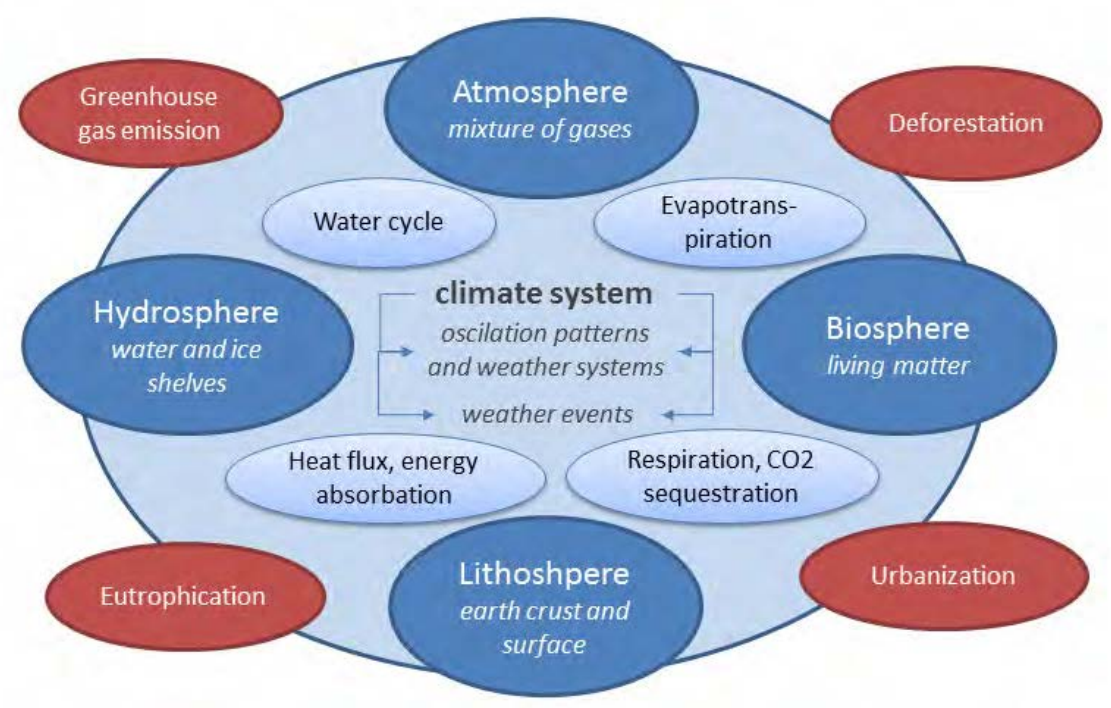

Figure 1.1. - The major components of the global climate system (WMO, 1966) and some major associated processes influencing global climate and weather systems. Each component influences, and in turn is influenced by, the entire system (Chapin et al., 2008) with stronger or weaker coupling. The disproportional emission of greenhouse gases changes the composition of the atmosphere and hence alters the current balance of the system (Chapin et al., 2008; IPCC, 2014). This can result in self-reinforcing feedback loops, for example melting of the polar ice-shelves or instability of tropical rainforest ecosystems (Lenton et al., 2008; Munson et al., 2018). Human activities (marked in red) affect balance in the system ("anthroposphere").

\subsubsection{Extreme weather events}

Following the definition of the World Meteorological Organization (WMO) weather is the "[...] state of the atmosphere at a particular time, as defined by the various meteorological elements", whereas climate is the long-term average of weather conditions in a specific area (WMO, 1966). The most immediate impact of climate warming on plant species is exerted by extreme weather events, for example severe storms with massive precipitation and associated windthrow, hail or landslides. Additional to these temporally and spatially restricted extreme weather events, persistence of weather events is expected to increase under climate warming (Francis et al., 2018; IPCC, 2014). This applies to drought and heat waves during summer months (Ummenhofer and Meehl, 2017), but also to cold waves during winter and early spring (Kreyling, 2010). Events such as the Central European heat waves of 2003 and 2018 stretch metabolism rates of individual plants to their limits, and can result in widespread mortality (Anderegg et al., 2013). They are also accompanied by proximate hazards, such as increased risk of wildfires (Balling et al., 1992; Turco et al., 2014), susceptibility to pest infestations, e.g. bark beetles in trees (Eatough Jones et al., 


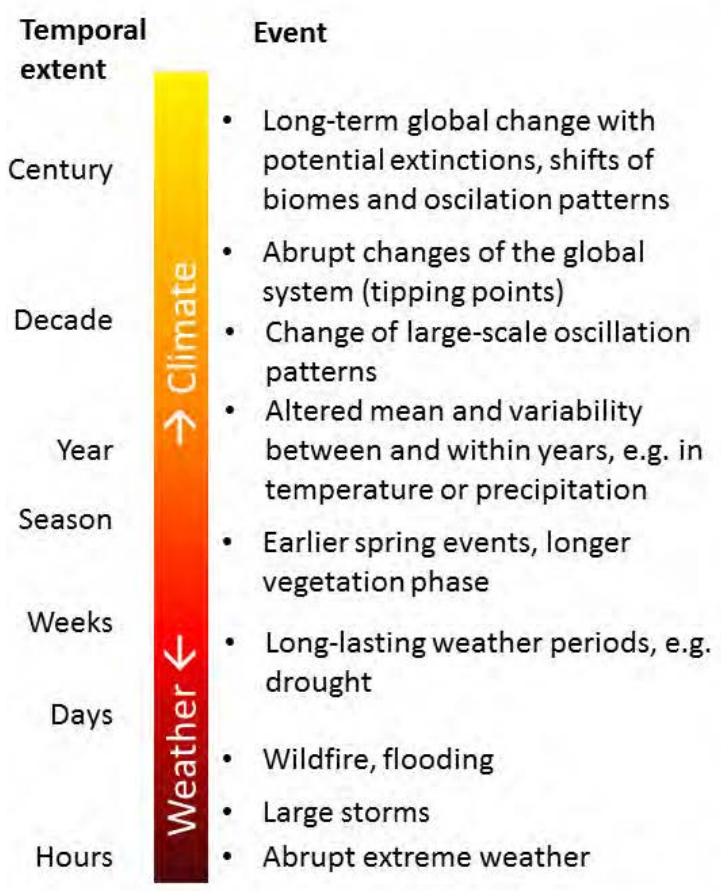

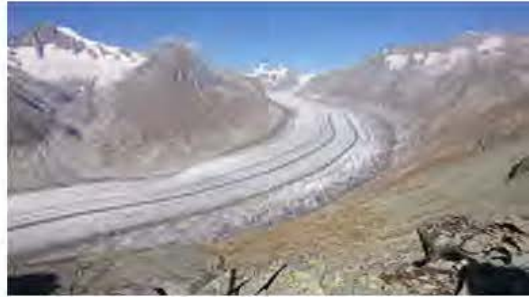

Vanishing glaciers became a symbol of climate warming. Here: Aletsch Glacier, Switzerland in October 2018 (own picture).

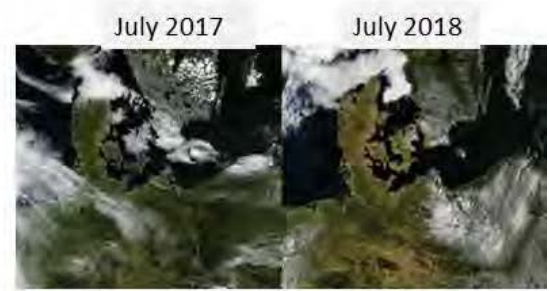

Comparison pictures reveal the impact of the 2018 drought on entire regions. Photos: NASA

Figure 1.2. - Temporal scales of expected climate warming impacts. Spatial extent of events usually corresponds with temporal extent, e.g. landslides from heavy precipitation events tend to be locally restricted whereas large oscillation patterns such as "El Niño" have a significant effect on a vast area. Abrupt changes on a global level are for example rapid decrease of permafrost in the taiga. Although "rapid" has to be understood in a geological context, thawing is expected to strike in a matter of decades or few centuries (Schuur and Abbott, 2011).

2004; Temperli et al., 2013), or pave the way for more pronounced negative impacts of shortterm extreme weather, such as increased risk for windthrow after drought (Peltola et al., 1999). Hence, the negative impact of extreme weather can be more or less pronounced depending on duration, frequency, preconditioning and seasonality of the event, as well as extreme values during the event (Ummenhofer and Meehl, 2017).

\subsubsection{Local climatic conditions}

For local populations of species or entire species ranges, long-term mean values, seasonality and variability of bioclimatic variables gain importance. These values describe the fundamental bioclimatic niche of the species (Bakkenes et al., 2002; Huntley et al., 1995). On a smaller scale, local populations can show adaptations, for example when comparing populations from the warmer range edge with populations from the colder range edge 
(Hampe and Petit, 2005; Rehm et al., 2015). Important bioclimatic variables for plants are for example precipitation regime and potential evapotranspiration, temperature minimum, maximum and mean levels, as well as onset and length of vegetation phase (Bakkenes et al., 2002). Inclusion of variability within and between years as well as seasonality, e.g. determining the wettest and driest quarter of the year, can further enhance the description of the climatic niche (Franklin et al., 2013). Additional to these climatic conditions, elevated $\mathrm{CO}_{2}\left(\mathrm{eCO}_{2}\right)$ levels can impact photosynthetic gas exchange and carbon assimilation, which directly affect plant growth (Gray and Brady, 2016).

Where climate warming alters prevailing local bioclimatic conditions beyond the bioclimatic niche of a given species, performance of its populations can be decreased to negative population growth and subsequent disappearance of the species from a given locality (Thuiller et al., 2005). However, response will be highly context-sensitive. For example, when Ernakovich et al. (2014) compared climatically related artic and alpine regions, they found higher productivity in arctic regions due to prolonged growing season while alpine communities are expected to decrease in productivity when earlier snowmelt decreases available water during the growing season.

Especially for long-term changes, i.e. alteration of conditions beyond the weather extremes of a year, community response and stability sets the context for the magnitude of species responses. The importance of community context is explained by the concept of species' fundamental and realized niche (McGill et al., 2006, see also fig. 1.3). The fundamental niche is defined by abiotic and bioclimatic conditions, the realized niche is co-determined by biotic interactions. On the extreme, species can be excluded from habitats within their optimum fundamental niche, for example by a stronger competitor or lack of beneficial interaction partners. Current climate warming is expected to alter species interactions, when response to bioclimatic changes differs between species. For instance, in a mixed stand of oak (Quercus petraea) and beech (Fagus sylvatica), oaks were less affected by drought than beeches, and subsequently experienced competitive release (Cavin et al., 2013). With increasing frequency of drought events, this might result in a shift of community composition where oaks exclude beeches. Similar observations were made in alpine meadow communities (Klanderud, 2005). Where changing climatic conditions trigger different response rates and strategies among species, interaction webs can be altered or disrupted. Reshuffling 


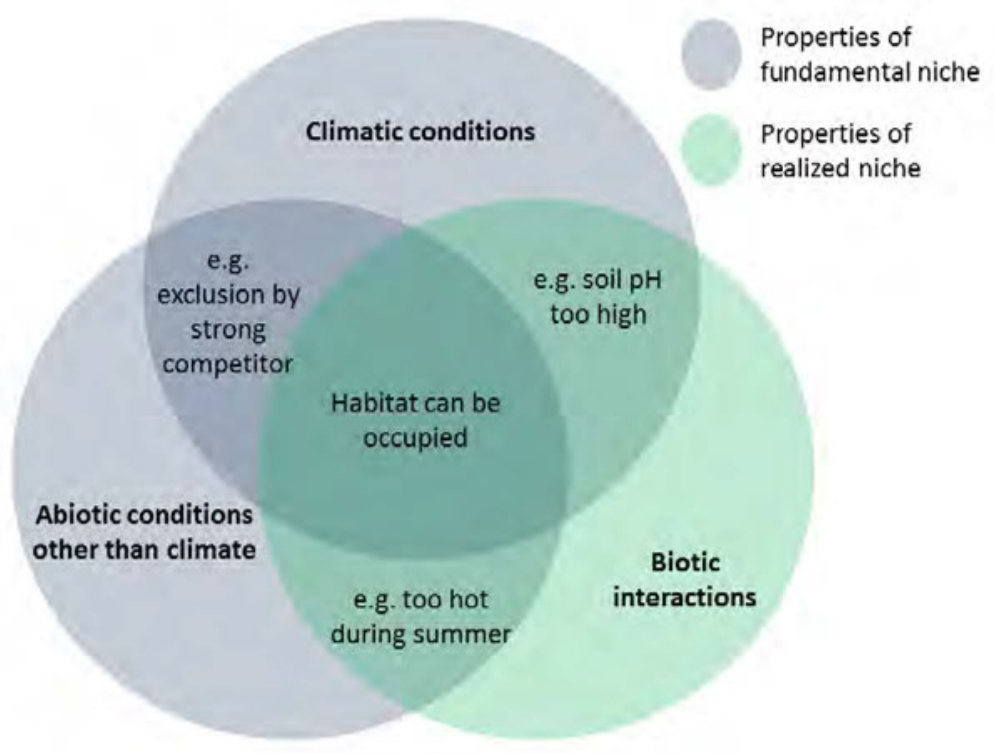

Figure 1.3. - Three determinants of habitat suitability. Climatic conditions, e.g. mean summer temperature, and other abiotic conditions, e.g. availability of nutrients, are properties of the fundamental niche of a species. Biotic interactions, e.g. competition or facilitation, determine whether establishment in an otherwise suitable habitat can be realized.

of community composition and interactions can result in decreased overall community stability and productivity with important consequences for ecosystem services (Zhang et al., 2018). For example, in the Tibetan Plateau, climate warming extended the stability of the dominant species, but decreased species richness and hence community asynchrony and decreased stability of biomass production through the vegetation period (Ma et al., 2017). Local warming can also affect interactions on a higher trophic level (Harvey et al., 2010; van der Putten et al., 2004), e.g. beneficial interactions with soil biota (Compant et al., 2010) or pollinators (Byers, 2017; Straka and Starzomski, 2014), but also release from enemies (Engelkes et al., 2008).

The accumulation of responses of local populations and communities can manifest on higher organizational levels, i.e. metapopulations and metacommunities, up to the level of entire species and biomes. Chapter 4 (pp. 75 to 104) contains an elaboration of such higher-level responses to climate warming. 


\subsection{Plant responses to climate change}

Vulnerability of species or populations to climate warming is determined by exposure to changing conditions, i.e. the magnitude and rate of locally changing conditions, but also the sensitivity of a species or population (Moritz and Agudo, 2013). How close is the population (already) to its physiological limits? How will changing interactions affect individual performance? To mitigate negative impacts, species response can be to adapt to hostile conditions or to escape from them. Extensive meta-analyses, analysing studies on 857 to 1598 species, comprising plants and animals in terrestrial and aquatic ecosystems, evidenced that between 40 and $76 \%$ of the studied species exposed long-term changes in phenology, range pattern, or morphology, and more than $80 \%$ of those changes were consistent with changing local climatic conditions (Parmesan and Hanley, 2015).

Escape or adaptation strategies are not mutually exclusive and plants can respond with a mixture of all response types (Bellard et al., 2012). In fact, change of physiological and morphological traits is often observed in the context of escape strategies, for example increased dispersal capability (Williams et al., 2016) or shift in phenology (Franks et al., 2007).

Similarly, range shift of phenotypes from warmer rear edges to more temperate zones of a species range can enhance adaptation potential and persistence of the receiving populations (Hampe and Petit, 2005; Rehm et al., 2015). In the following, I will focus on physiological and morphological changes that enhance a species' persistence in situ and range shift promoting persistence ex situ.

The response strategy of a species is determined by the adaptive potential of the species as well as its ability and possibility to escape. Where the species-specific strategy cannot keep pace with rapidly changing bioclimatic regime and associated community effects, populations are deemed to go extinct on the long term (Cahill et al., 2013; Urban, 2015).

\subsubsection{In situ response}

Changes in morphology or physiology expressed as plastic or evolutionary responses to novel climatic conditions can aid a local population to persist in changing bioclimatic 
conditions. Plastic response (phenotypic shift) is triggered by immediate environmental conditions (Hoffmann and Sgrò, 2011; Nicotra et al., 2010), i.e. "[...] the ability of a genotype to express a different phenotype [...]" (Franks et al., 2014). Evolutionary changes (genotypic shift) manifest in the genetic structure of a population (Hoffmann and Sgrò, 2011). Whether change of the phenotype is related to plasticity or evolution is difficult to tell apart (Franks et al., 2014). For the scope of this overview, I will treat plastic and evolutionary responses equally and focus on the expression of changes.

Shifts in phenotype or genotype can be considered adaptive if they increase individual performance or prevent decrease of performance in changing conditions (Franks et al., 2014; Nicotra et al., 2010) and mitigate climate warming-induced stress. This stress can be caused by rising temperatures and altered precipitation regime, but also by elevated $\mathrm{CO}_{2}$ level in the atmosphere (Becklin et al., 2016; Gray and Brady, 2016). As such changes allow the species to persist under novel bioclimatic conditions they can be considered as bioclimatic niche shift. For example, morphological changes were observed for leaf width of Dodonaea viscosa subsp. angustissima in Austraila, decreasing towards the equator (Guerin et al., 2012). Herbarium data confirmed a constant change for the past 127 years and recorded a warming of $1.2^{\circ} \mathrm{C}$ during that period. The same study also revealed a negative correlation of leaf area with altitude. The changes might be related to maximum temperature (leaf width, latitude) and minimum temperature (leaf area, altitude). Further responses can affect root:shoot ratio, root biomass and architecture as response to drought and heat stress (as reviewed by Gray and Brady, 2016), or the shift of flowering time. Earlier onset of flowering can be enhanced by increasing mean temperature during winter and spring (Mohandass et al., 2015) or number of frost-free days (Park and Mazer, 2018). Another common predictor for shift of flowering onset is maximum summer heat peak, causing earlier flower onset for some species, but also a delay of flowering into post-heat time (Sherry et al., 2007). However, these responses to novel climatic conditions come at a cost. For example, Scheepens and Stöcklin (2013) transplanted high-elevation phenotypes of Campanula thyrsoides L. to sites at lower altitudes to test for plastic response under climate warming. The plants showed a clear correlation between earlier phenology and lower elevation, i.e. higher temperatures and lower precipitation. However, they also showed decreasing seed production along the down-slope gradient. On the long term, this response might result in overall population decline and hence manifest as maladaptation to 
The fate of plant species under climate warming

climate warming (Scheepens and Stöcklin, 2013).

Different types of the driving force, i.e. heat or drought stress, require different adaptations and are reported to trigger different, sometimes opposing responses (Becklin et al., 2016; Franks et al., 2014; Gray and Brady, 2016). As a further complication, it is very likely that different drivers act simultaneously and can counteract each other with a mitigation of negative effects or limiting positive effects, depending on the context. For example, drought and heat can inhibit growth enhancement under $\mathrm{eCO}_{2}$ conditions (Reich et al., 2014), but $\mathrm{eCO}_{2}$ can similarly increase the recovery from heat and drought damage after the stress peak (Roy et al., 2016). Other studies confirm a difference between functional groups, i.e. legumes in comparison with grasses, in simultaneous effects of $\mathrm{eCO}_{2}$ and drought stress (AbdElgawad et al., 2015).

Additional to interactions of the bioclimatic drivers, i.e. temperature, precipitation and $\mathrm{eCO}_{2}$, further environmental conditions can critically influence the response capacity of a species and even switch the signs of its response. The study by Reich et al. (2014) was part of the long-term experiment BioCON, which was started in 1997. In this experiment, observations of response of $\mathrm{C} 3$ and $\mathrm{C} 4$ plants to $\mathrm{eCO}_{2}$ and $\mathrm{N}$ initially delivered the expected result of higher biomass production under $\mathrm{eCO}_{2}$ in $\mathrm{C} 3$, but not in $\mathrm{C} 4$ plants (Reich et al., 2018). However, this pattern was inverted after eight years, where C4 plants had a higher biomass production in $\mathrm{eCO}_{2}$ environments than under ambient conditions, while $\mathrm{C} 3$ plants no longer benefitted from $\mathrm{eCO}_{2}$. Reich et al. (2018) attribute this effect to increased $\mathrm{N}$ supply (but see Nie et al., 2018). Assuming further increase of global N deposition (Kanakidou et al., 2016), such insights highlight the importance of holistic experimental and analytical approaches to predict the impact of environmental drivers on plant species.

Hence, to assess the adaptive capacity of a species, in plastic as well as evolutionary responses, it is necessary to set observations in context with simultaneously operating bioclimatic and environmental drivers, as well as community and species-inherent variables such as functional group, gene pool size and diversity. Further long-term research is required to find out whether those responses occur fast enough to keep pace with rapid climate warming and prove to be sustainably adaptive (Franks et al., 2014; Jump and Penuelas, 2005). 


\subsubsection{Range dynamics}

Range dynamics, i.e. the shift of distribution patterns, has been observed not only for plant species, but through all taxa (Parmesan and Hanley, 2015; Parmesan and Yohe, 2003; Root et al., 2003; Walther et al., 2005). During range shift, populations of a species establish in habitats beyond their former range border. The zone of expansion is often called "leading edge". The opposite "trailing edge" is characterized by disappearance of populations of that species. The ratio of colonialization on the leading edge and extinction on the trailing edge can result in net gain of range area (range expansion), net loss of range area (range contraction), or equilibrium (range shift, see also fig. 4.2). Classically, establishment of novel populations is the main focus of the respective studies, i.e. regarding dynamics on a species level. To assess the potential of adaptation of populations, it is very advisable to observe "intra-range shifts" as well. That is the shift of the genotype of a species, e.g. from more extreme range edges to more temperate zones (Hampe and Petit, 2005; Rehm et al., 2015), but also abundance shifts. Where range shift into novel habitats ("marching") cannot be realized, the current range borders may remain while overall abundance of the species within the current range ("leaning") shifts along with climate warming (Breshears et al., 2008). The prevailing directions of climate warming-induced range border shift are towards higher latitudes and altitudes (Parmesan and Hanley, 2015; Parmesan and Yohe, 2003; Thomas, 2010, but see Fei et al., 2017; Lenoir et al., 2010).

Related, and often discussed together, is the introduction of species from remote distances, e.g. different continents, which by definition is a range expansion process as well. Such species were introduced by anthropogenic means, either unintended as "blind passengers" in global trade and travel, or intentionally for economic or decorative reasons (Hulme, 2007; Meyerson and Mooney, 2007). It is highly unlikely that those species could have reached the remote location without human aid. In macroecology, exchange between such otherwise disconnected locations is termed "teleconnection" (Heffernan et al., 2014). Research on climate warming-induced range shifts is largely inspired by concepts from invasion biology, where intercontinental range expansion is examined. For the scope of this overview, I will only refer to species that shift their range within their projected dispersal envelope. I explicitly exclude species from remote distances, although I acknowledge their potential immense impact on native species and communities (Bauer, 2012; Walther et al., 2009). 
While mobile species, i.e. animals, can actively follow their climatic niche, range expansion of plants follows to a certain degree more stochastic rules in a sense of non-directed dispersal of propagules. This implies that propagules of a species have very likely been dispersed to that novel site before, but establishment of a local population was not successful, i.e. the locality was outside of the species' fundamental or realized niche (see fig. 1.3). Under climate warming, bioclimatic limitations on such a site may be relaxed, e.g. through increasing mean temperature or precipitation, and hence allow for colonialization. The rate of bioclimatic change can affect the velocity of species range shifts. For example, when comparing range shift rates of tree species in three locations in Canada, Becker-Scarpitta et al. (2019) found no significant changes in elevational distribution and species richness in the site with the weakest recordings of bioclimatic changes, but high rates of elevational shifts and a significant decrease of species richness in the site with highest bioclimatic changes.

Not only velocity, but also direction of range shift can be determined from climatic conditions. A study observing 86 tree species, found a strong relation between the prevailing direction of range shift and species' drought tolerance and functional type (Fei et al., 2017). The auhors observed westward and poleward shifts, but species with lower tolerance to drought were almost fully excluded from westward shifts, where available moisture was a limiting factor.

Beyond abiotic conditions, biotic interactions are an important factor for the realization of novel population establishment. When climate warming alters local bioclimatic conditions, species arriving from more extreme climate zones may be preadapted to the locally novel conditions and hence have an evolutionary advantage over resident species. For example, Alexander et al. (2015) used an altitudinal transplanting approach to simulate success or failure of niche tracking through migration. They found that pre-adapted novel competitors had significant negative impacts on the transplanted focal species (failure scenario), whereas the effect of novel competitors was negligible when the focal species was pre-adapted (success scenario).

Competitive interactions might also be a reason why some species move "against the trend", for example when some species of a pool shift to lower instead of higher altitudes. Lenoir et al. (2010) proposed that this might be related to climate warming-induced decrease of competitor performance that had before excluded the downward-shifting species from 
otherwise suitable habitats.

Diverging response rates of species from the same pool can lead to disruption of ecological networks during range shifts, shaping new communities. For example, from a mixed stand pool, Fei et al. (2017) observed a stronger westward expansion for angiosperms and a stronger poleward expansion for gymnosperms, resulting in forest types that differed strongly from the initial type during range shifts. Disruptions can also occur on higher trophic levels, e.g. with mutualistic species (Caddy-Retalic et al., 2018; Ehlers, 2011; Schweiger et al., 2008), but also enemies (Engelkes et al., 2008). Respecting biotic interactions on the same and higher trophic levels is crucial for realistic estimations of species persistence and abundance under climate warming (Van Der Putten et al., 2010). In fact, on a local scale, biotic interactions may even have a higher impact on individual performance than climatic variables (Tomiolo et al., 2015).

Before any establishment attempts, species must reach a novel habitat. To reach novel habitats in time will be increasingly difficult in modern times due to anthropogenic landscape fragmentation (Cobben et al., 2012; Honnay et al., 2002; Opdam and Wascher, 2004). To escape current climate warming, plants would need to move on average by $1 \mathrm{~km} /$ year (Corlett and Westcott, 2013). But even in un-fragmented landscapes or under assisted dispersal, poleward range shift can be spatially limited by photoperiod (Saikkonen et al., 2012) or, more profoundly, by reaching a lands' end such as a mountain summit or an ocean.

\subsubsection{Extinction}

Potential species loss through climate warming-related extinctions is estimated to accelerate with increasing temperature rise (Urban, 2015). The rate of global warming is expected to be higher in tropical lowland and subtropical regions as compared to higher altitudes and temperate zones (Loarie et al., 2009; Williams et al., 2007), with a correspondingly higher risk of extinction of species (Urban, 2015). High species loss in the Amazonian lowlands and rainforest might result in increasing instability of the entire rainforest ecosystem which itself is a tipping point of self-reinforcing climate warming (Lenton et al., 2008). Extinctions may ultimately be related to the actual rise of temperatures, the failure to 
adapt or maladaptation (e.g. Scheepens and Stöcklin, 2013), but also by more proximate processes such as loss of crucial interaction partners or suppression by species that are better adapted. Hence, it is difficult to put actual extinction risk through climate warming in definite numbers. To date, 20 extinctions have been declared to be directly attributed to climate warming (Cahill et al., 2013) - however, these are only the extinctions we detected by now and, under future projections of climate warming, they are only the tip of the rapidly melting iceberg (Urban, 2015).

\subsection{Discussion - How to predict species response to climate warming?}

In this overview, I summarized how climate warming affects plant species and how they can mitigate negative effects of climate warming. Understanding the impacts of climate warming and associated threats to species is necessary for pro-active conservation management (Dawson et al., 2011), such as assisted migration (Gallagher et al., 2015; Vitt et al., 2016) and measures to increase gene flow between populations (Hoffmann and Sgrò, 2011), but also for preventing undesirable climate-enforced invasions of harmful species (Bauer, 2012), decreased ecosystem stability and loss of ecosystem services (García-Palacios et al., 2018). Climate warming, the expected bioclimatic changes and the species responses to those changes are a highly complex system with many unknown variables and interactions of individual elements.

A key to assess species response might be a trait-based approach. Functional traits indicate habitat requirements of a species (Belluau and Shipley, 2018) and therefore can serve as proxies to assess the sensitivity of a species to expected local bioclimatic changes. Fei et al. (2017) were able to relate different directions of tree range shifts to dispersal mode (animal or wind dispersed), as well as drought tolerance of the tree species. Trait-based approaches promote the transferability of observations, as they have an inherent generality (McGill et al., 2006). Traits can be a key determinant of community composition with strong correlation to fine-scale drivers such as successional stage, disturbance regime and biotic interactions (Bruelheide et al., 2018; Kunstler et al., 2016). Changes in trait combinations within communities can give valuable information on local community stability (Májeková et al., 2014) and may be the base for an early-alert of system transitions (Dakos et al., 2019). 
However, traits are only meaningful when embedded in a broader context (MacLean and Beissinger, 2017). Despite a general consistency of explanatory traits for plant abundance (Bruelheide et al., 2018), single or few traits may not be significant for the question at hand (Küster et al., 2008). For explaining invasive success of species, a shift of explanatory power of traits over time has been shown where traits that facilitate colonialization in a novel habitat may not necessarily be predictors for long-term persistence (Catford et al., 2019; Kempel et al., 2013). Therefore, it can be reasonable to decompose the overall problem into smaller steps, answering the fundamental questions: How do local changes in climatic conditions affect a focal species, how can that species mitigate bioclimatic stress, and how do biotic interactions affect individual performance? For each of these questions, different sets of traits will likely have the greatest explanatory power, as they emerge from different underlying processes. Estrada et al. (2016) have proposed such a framework, decomposing range shift into distinct stages and proposed some general trait categories relevant for each stage, including for example movement ability, ecological generalisation, persistence under unfavourable conditions and competitive ability. This framework was constructed to be applied to animals and plant species alike and might also be used in a multi-species context, for example when studying concurrent range dynamics of plant species and their mutualistic or antagonistic interaction partners on other trophic levels. Further implementation of environmental stress locally expected from climate warming and species mitigate that type of stress can additionally enhance such a framework, allowing for capturing niche loss and shift.

Recently, advances in big data handling and accessibility of large data repositories have opened the door for large-scale analysis of species traits. For example, Bruelheide et al. (2018) used data on 26,000 species to investigate the relationship of trait composition, community interactions and environmental filtering. Combining this wealth of information with contemporary climate information may help to establish more accurate predictive models for assessing species responses to climate warming (see also chapter 4). 



\title{
2. Influence of seed size on performance of non-native annual plant species in a novel community at two planting densities
}

\author{
This chapter was published under the same title in Acta Oecologica, October 2018. \\ Full author list: J Radny , KM Meyer, K Tielbörger, WH van der Putten

\section{Declaration of contribution:} \\ $J R, K M$ and WP designed the study, KT provided seeds and expertise, JR implemented the \\ experiment, JR and KM analyzed the data. For this published version, KM implemented \\ data analysis and generated statistical figures. JR and KM wrote the first draft of the \\ paper, all authors worked on all subsequent versions of the paper. Overall, this paper was \\ submitted to four different journals with increasing improvements based on anonymous \\ reviewer's suggestions.
}

Figures 2.2 to 2.4 with statistical content are similar to those published. Figures 2.1 and 2.5 with photographic documentation of the experiment were not part of the original publication. Referenced literature is included in the full thesis literature.

\footnotetext{
Abstract

Climate warming enables plant species to migrate to higher latitudes and altitudes. Within Europe, the Mediterranean harbours many species that might expand their ranges towards Western Europe. Small seed size may facilitate dispersal, however, it may impair establishment of the range-expanding plant species in the novel vegetation. In a greenhouse
} 
experiment, we examined effects of average seed size of Mediterranean plant species on their establishment in a mixed community of Western European plant species. Applying two levels of densities of the natives and a herbivory treatment, we tested how seed size is linked to response in plant growth and fitness in novel vegetation. While all non-native plant species showed a negative response to increased planting density, species with small seeds showed a less negative response. This effect persisted under herbivory. Our data suggest that small-seeded non-native plant species may tolerate competitive pressure from novel plant communities better than large-seeded species, so that small seed size may confer a higher probability of establishment of non-native species in novel communities.

\subsection{Introduction}

Global warming has caused range shifts of many plant and animal species to higher latitudes and altitudes and leads to the introduction of many non-native propagules into native recipient communities (Parmesan and Yohe, 2003; Root et al., 2003; Walther et al., 2005). Dynamics of such range shifts differ among species (le Roux and McGeoch, 2008). Drawing back on concepts of invasion biology, these differences are a result of variation in dispersal capacity and establishment success in a novel habitat (Hampe, 2011; Richardson and Pyšek, 2012). Predominantly, altogether habitat suitability determines initial establishment of plant individuals (Gerhardt and Collinge, 2007; le Roux and McGeoch, 2008).

Negative biotic interactions with the recipient community, i.e. biotic resistance, form a second obstacle to the successful establishment of plant individuals (Kempel et al., 2013; Parker and Hay, 2005; Taylor et al., 2016) and the formation of a new satellite population in a habitat beyond the former range. As part of the biotic resistance of a recipient community, competition is a major mechanism to inhibit the intrusion of novel species (Caño et al., 2007; Moorcroft et al., 2006; Svenning et al., 2014).

However, relatively little is known about the degree to which competition may inhibit establishment of range-shifting plant species in novel plant communities, especially because of the difficulty to detect plant invasions that failed (Zenni et al., 2014). While it seems intuitive that interspecific competition may drastically decrease establishment success, it is probably not sufficient for inhibiting the establishment of novel species (Levine et al., 
2004). In spite of the considerable support for the existence of biotic resistance in some studies, an increasing amount of studies question the role of biotic resistance in slowing down plant invasions (Jeschke et al., 2012; Levine et al., 2004).

Studies often are difficult to compare as they are dealing with a variety of native and non-native species, showing a vast spectrum of species-specific traits. Community ecology has emphasized the importance of traits in formation of a local community (McGill et al., 2006; Thuiller et al., 2012). It might therefore be exactly these specific traits that determine whether or not a non-native species is able to successfully establish among novel neighbours.

We examined whether the establishment of non-native species with different traits is affected by an increased biotic resistance in the form of increased intensity of competition conferred by native plant mixtures of different planting densities. We also applied a herbivory treatment to account for potential interactions between native plant density and herbivory in affecting non-native plant performance. We chose seed mass within a plant family as a trait expression that can be relevant for the response to increased planting density. Former studies have shown a positive relationship between seed size and seedling survival, as well as tolerance to hazards (Leishman et al., 2000; Metz et al., 2010; Westoby et al., 1996). Moreover, large seeds produce large plants that can reach above- and belowground resources better and faster than smaller neighbours (Jakobsson and Eriksson, 2000; Leishman, 2001; Westoby et al., 1996), which is expected to enhance their ability to capture resources in direct competition with natives (Schwinning, 1996; Schwinning and Weiner, 1998). Based on earlier findings (Dostál, 2011; Hierro et al., 2013), we tested the hypothesis that non-native species with large seeds will show a smaller negative response to increased density of natives than nonnative plant species with small seeds. Further, based on Kempel et al. (2013), we tested the hypothesis that herbivory will at least weakly reduce non-native plant performance. Overall, we expected increasing seed size to enhance establishment in novel vegetation. 


\subsection{Material and methods}

\subsubsection{Study organisms}

The non-native species used in this study were annuals native to the Eastern Mediterranean (table table 2.1) and species selection was based on the long-standing experience with the flora of the region of one of the authors (KT). Currently, many plant species from Mediterranean Europe are spreading to higher latitudes (Tamis et al., 2005; Walther et al., 2002).

Nowadays, Mediterranean species are reported to comprise $21 \%$ of the non-native flora in Switzerland (Wittenberg et al., 2006). Taking all non-native species from the same source pool allowed us a better estimation of the net trait effect (Pyšek and Richardson, 2007).

In May 2011, ripe seeds were collected in Israel from several populations and shipped to our lab. Most non-native species used in the experiment do occur at least casually in several European countries and are partly listed as established aliens (table 2.1), indicating that these species would generally be able to survive in Western Europe. We used seeds from the original range in Southern Europe in order to ensure that they have no evolutionary history in the novel range (Pyšek et al., 2009; Richardson and Pyšek, 2012). In order to control for the influence of plant family, we chose species from two families, Asteraceae and Poaceae, which are species-rich and widespread and are reported to harbour many species with a weedy tendency (Lambdon et al., 2008), making them potential candidates for range shifts (Tamis et al., 2005).

As native species, we chose two grass species (Agrostis capillaris L. and Festuca rubra L.) and two forb species (Hypericum perforatum L. and Plantago lanceolata L.). Earlier studies confirmed their wide distribution in Western Europe (Roscher et al., 2004). Seeds of native species were collected in the Netherlands.

\subsubsection{Experimental design}

We carried out the experiment in our greenhouse in Wageningen, NL, in 2011. We germinated seeds of all individual species separately on glass beads in a germination chamber at $22^{\circ} / 16^{\circ} \mathrm{C}$ (day/night) with a day length of $16 \mathrm{~h}$, representing early summer conditions in 
Table 2.1. - List of non-native species used in the experiment. Initial seed sizes were determined directly as seed mass from the collected seeds and were averaged over 20 seeds. For the dimorphic species $C$. sancta, we used the heavier peripheral seeds in the experiment. Reports of establishment status is based on European Invasive Species Gateway (DAISIE, 2012). Note that several species are also reported as "not established" or "casual occurrence" in further European countries.

\begin{tabular}{|c|c|c|}
\hline Species & $\begin{array}{l}\text { Initial seed size, } \\
\text { mean } \pm \text { standard } \\
\text { error of the mean } \\
(\mathrm{mg})\end{array}$ & $\begin{array}{l}\text { European countries where } \\
\text { this species is an established } \\
\text { non-native species }\end{array}$ \\
\hline \multicolumn{3}{|l|}{ Asteracea } \\
\hline $\begin{array}{l}\text { Chrysanthemum coronarium } \\
\text { (L.) } \\
\text { Cass. ex Spach }\end{array}$ & $2.03 \pm 0.13$ & Azores, France, Madeira \\
\hline Crepis sancta (L.) Babcock & $0.27 \pm 0.005$ & $\begin{array}{l}\text { Luxembourg, Switzerland, } \\
\text { Spain }\end{array}$ \\
\hline Filago palaestina Boiss. & $0.10 \pm 0.01$ & Azores, France, Madeira \\
\hline Silybum marianum Gaertn. & $28.38 \pm 0.86$ & $\begin{array}{l}\text { Azores, Denmark, Great } \\
\text { Britain, Ireland, Sweden }\end{array}$ \\
\hline \multicolumn{3}{|l|}{ Poaceae } \\
\hline Avena sterilis (L.) & $45.63 \pm 2.24$ & Azores, Great Britain \\
\hline Aegilops ovata (L.) & $34.01 \pm 4.40$ & Not established \\
\hline Bromus fasciculatus (C. Presl) & $1.25 \pm 0.05$ & No data available \\
\hline Stipa capensis (Thunb.) & $3.31 \pm 0.17$ & Casual occurance \\
\hline
\end{tabular}

North- Western Europe. Pre-germination was necessary to ensure sufficient plant individuals per pot at the onset of the experiment, in order to isolate the direct effect of seed size and not its indirect effect via germination times or germination success. Thus, to obtain seedlings of similar size, we stored them upon germination until planting at $4^{\circ} \mathrm{C}$ at high light, to inhibit growth. We started the experiment when at least 50 seedlings of each non-native species and 700 seedlings of each native species were available. We planted the seedlings in pots of $18 \times 18 \times 22 \mathrm{~cm}$ with a volume of about $7 \mathrm{~L}$, filled with sandy-loamy soil collected in a close-by riverine area where all four native plant species occurred. Soil was sieved to remove most seeds and medium-sized soil organisms, e.g. earthworms and insect larvae, and homogenized. The live status of the soil enabled interactions with the microbiological soil community from the range of the native species.

Each pot was planted with four individuals of one non-native species as well as either 12 or 44 native individuals, creating low or high density, respectively (fig. 2.1). We planted either three or eleven individuals per native species in a pot, so that each of the four native species contributed in equal shares to the community mixture. By increasing the number of natives only and keeping the number of non-native individuals constant, potential 


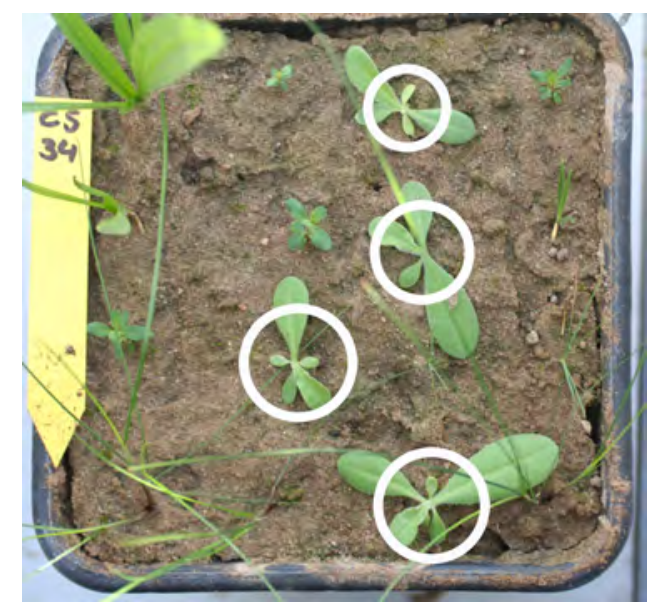

(a) Low density with 16 plants.

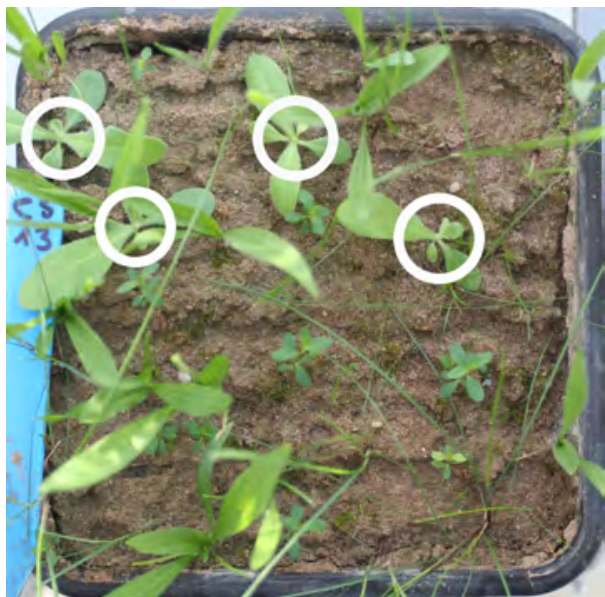

(b) High density with 48 plants.

Figure 2.1. - Pots with Crepis sancta as non-native species, about 30 days after planting. White circles indicate the non-native individuals.

intraspecific competition among non-natives stayed at a constant level, thus facilitating the focus on interspecific competition originating from different densities. Planting followed a $4 \times 4$ (low density) or $7 \times 7$ (high density) grid in each pot, in order to avoid clumping effects. Position of individuals along the grid was randomized.

Half of the pots were subjected to herbivory by the insect species Locusta migratoria and Mamestra brassicae. We chose these herbivores for their wide distribution within Europe $(C A B I, 2013)$ and their generalist feeding behaviour (Macel et al., 2005). Herbivores were added consecutively for one week per species. First we added three individuals of $L$. migratoria to the respective pots and removed them after one week of feeding time. We then visually inspected all plant individuals for feeding traces on leaves and seeds. This procedure was then repeated with three individuals of $M$. brassicae.

Greenhouse climate was adjusted to early summer conditions in the European species range $\left(60 \% \mathrm{RH}\right.$; day $21 \pm 2^{\circ} \mathrm{C}$; night $16 \pm 2^{\circ} \mathrm{C}$; $16 \mathrm{~h}$ of light with $\left.250 \mu \mathrm{mol}{ }^{-1} \mathrm{~m}^{-2} \mathrm{PAR}\right)$. We watered the pots with tap water every other day. During the first ten days, dead seedlings were replaced by new ones from stock. Later on, we considered death of an individual to be a natural part of the experiment rather than a side-effect of experimental manipulation such as root damage during planting.

For each species $\mathrm{x}$ treatment combination we had three replicates, yielding 2 families $\mathrm{x}$ 
4 species $\times 2$ competition treatments $\times 2$ herbivory treatments $\times 3$ replicates $=96$ pots, having 384 non-native and 2688 native individuals overall. In each pot, above-ground plant biomass was harvested when nonnative individuals had produced ripe seeds and showed first signs of senescence. The experiment lasted 15 weeks. Shoots were dried for $48 \mathrm{~h}$ at $70^{\circ} \mathrm{C}$ and weighed separately for each species per pot to determine dry shoot biomass. We also measured the length of the longest shoot for each non-native species for Poaceae up to the last seed and for Asteraceae up to the lower end of the flower head. We determined seed number as a proxy of plant fitness. The average weight of 20 seeds was used for extrapolating total seed number from total seed weight.

\subsubsection{Statistical analysis}

We assessed the relationship between the response variables shoot biomass and length of longest shoot, respectively, and the explanatory variables competition treatment (high or low native plant density), herbivory treatment (with and without herbivory), family (Poaceae or Asteraceae), the continuous variable "average initial seed size" and their interactions with linear ANCOVA-type models.

After successfully checking that model assumptions were not violated, models were simplified by manually removing the largest non-significant interactions one after the other until only variables with at least one significant effect at $p<0.05$ were left in the model. For the final shoot biomass model, one outlier was removed, which improved the Akaike Information Criterion. For number of seeds as a response variable, model assumptions of neither linear models nor generalized linear models were met. Therefore, we applied the loess-function as non-parametric smoother of the relationship between number of seeds and initial average seed size separately for high and low native plant density, with and without herbivory, and for the two plant families. To compare competition effects between families, relative interspecific effects of density were calculated as

$$
\frac{\mu_{\text {high }}-\mu_{\text {low }}}{\mu_{\text {low }}}
$$

(Steidl and Thomas, 2001), where $\mu$ stands for the average performance measure and the indices for high and low density of the native species mixture. More negative values indicate 
stronger competitive effects of natives on non-natives. We calculated relative effects of density for three performance measures, i.e. shoot biomass, length of longest shoot and number of seeds. These effects were calculated for Poaceae and Asteraceae species and for all species pooled per family.

Statistical analyses were conducted with R version 3.1.3 (Team, 2016), using the ggplot2 package (Wickham, 2009).

\subsection{Results}

Seed size generally was a good predictor of biomass under novel conditions (fig. 2.2, table 2.2). Contrary to our expectations, high planting density decreased shoot biomass much more strongly at larger seed sizes than at smaller seed sizes (fig. 2.2, table 2.2: Initial seed size: Competition treatment interaction). For Poaceae, seed size was also generally positively correlated with shoot length, but this correlation was negative for Asteraceae ( fig. 2.3, table 2.2: Initial seed size: Family interaction). Seed size affected the number of seeds in a hump-shaped form, peaking at smaller than medium seed sizes (fig. 2.4).

The competition treatment high planting density markedly decreased shoot biomass, shoot length, and number of seeds of the non-natives (figs. 2.2 to 2.4, tables 2.2 and 2.3). Despite visible herbivore damage on most species, at time of harvest herbivory did not have a significant impact on non-native plant performance. Overall visible damage was very low for Asteraceae species ( $<15 \%$ of leaf area), whereas up to $90 \%$ of the seeds of the grass species were consumed by locusts. The only exception was $A$. ovata which had not produced seeds by the time we added locusts to the pots (see peak in the dotted curve in fig. 2.4). There was a significant family effect: All three response variables on average showed higher values for Asteraceae than for Poaceae (figs. 2.2 to 2.4, tables 2.2 and 2.3). In terms of mortality, only four of 144 non-native plant individuals died during the experiment, with no obvious relation to species, herbivory or planting density. 


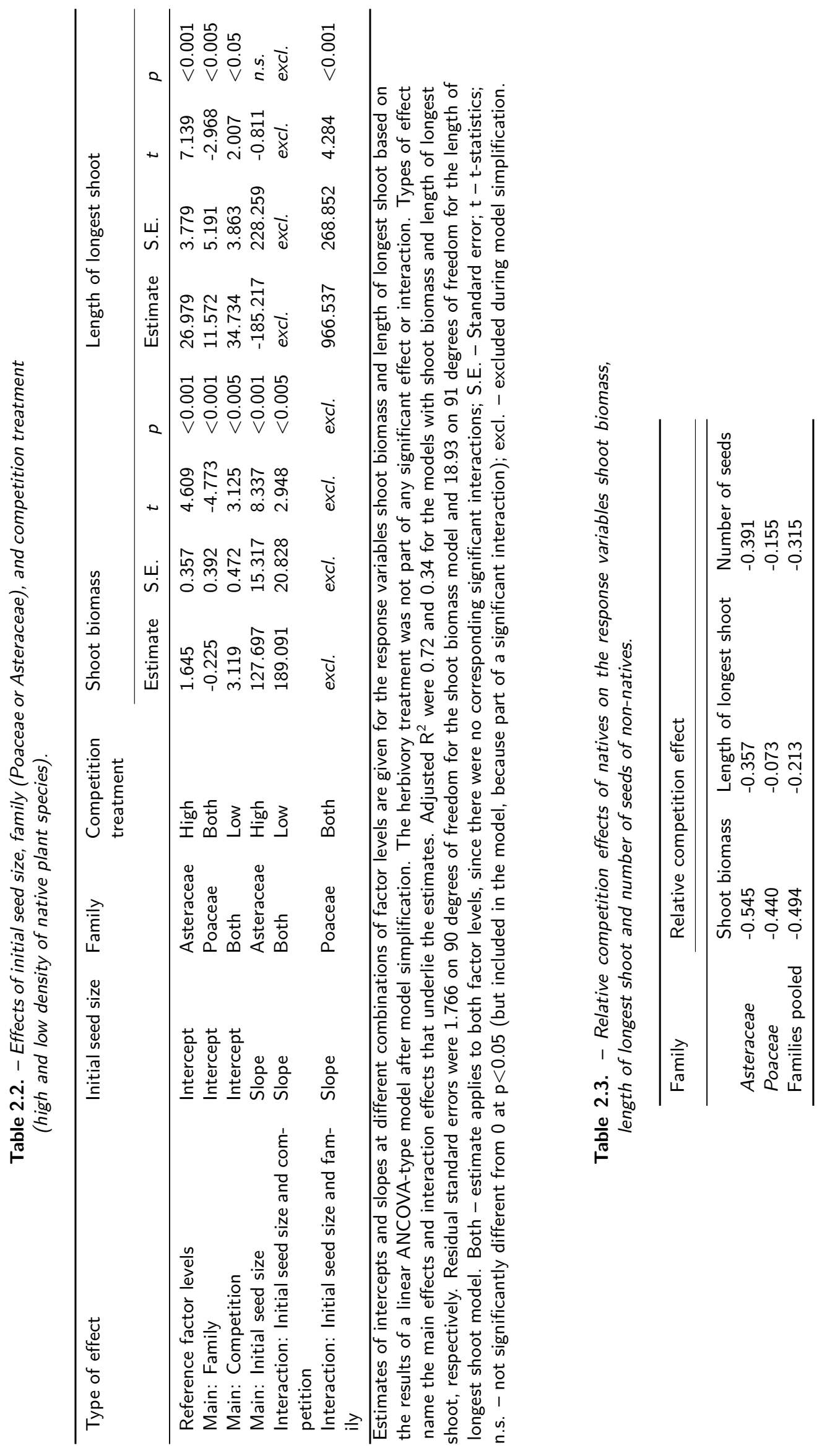




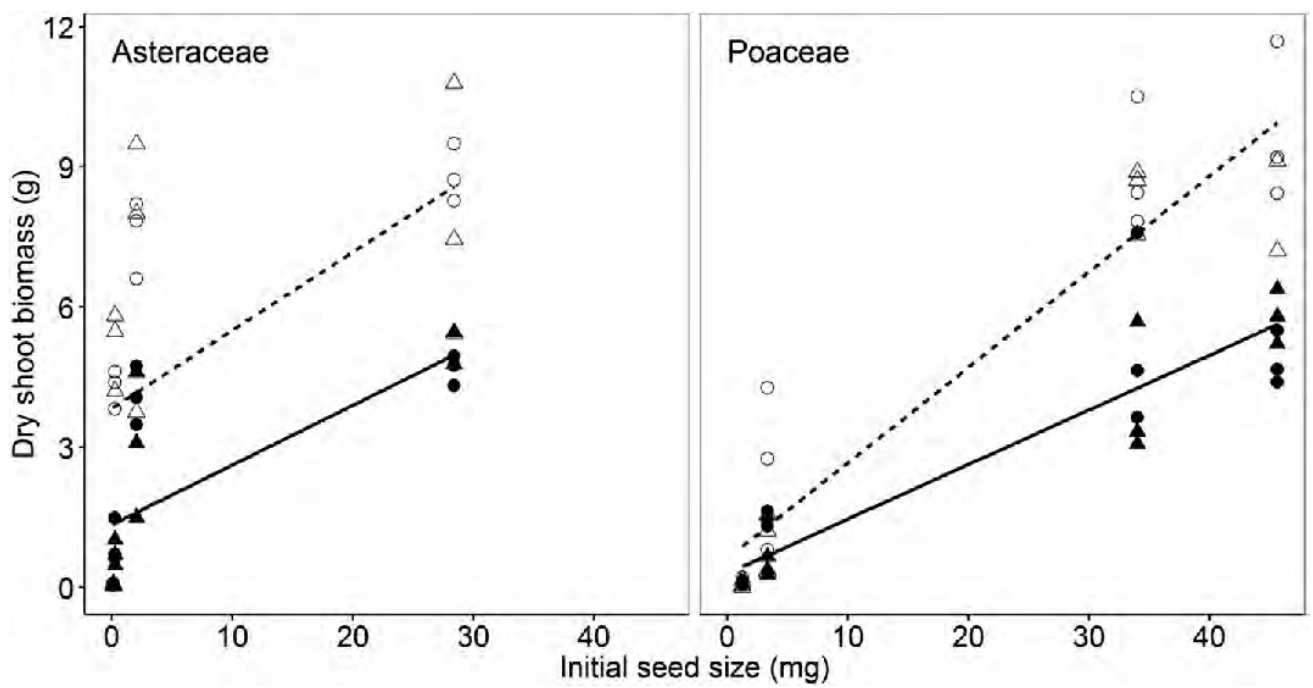

Figure 2.2. - Asteraceae species (left panel) and Poaceae species (right panel) showed increased dry shoot biomass per pot with increasing seed sizes. This increase was weaker at high (solid symbols, solid line) than at low (open symbols, dashed line) planting density of native species, especially for Poaceae. Dry shoot biomass did not significantly differ with herbivory (triangles) and without herbivory (circles). Each species is represented by its average initial seed size illustrated here by a vertical stack of points each. Lines represent significant linear regressions (see table table 2.2 for complete statistics).

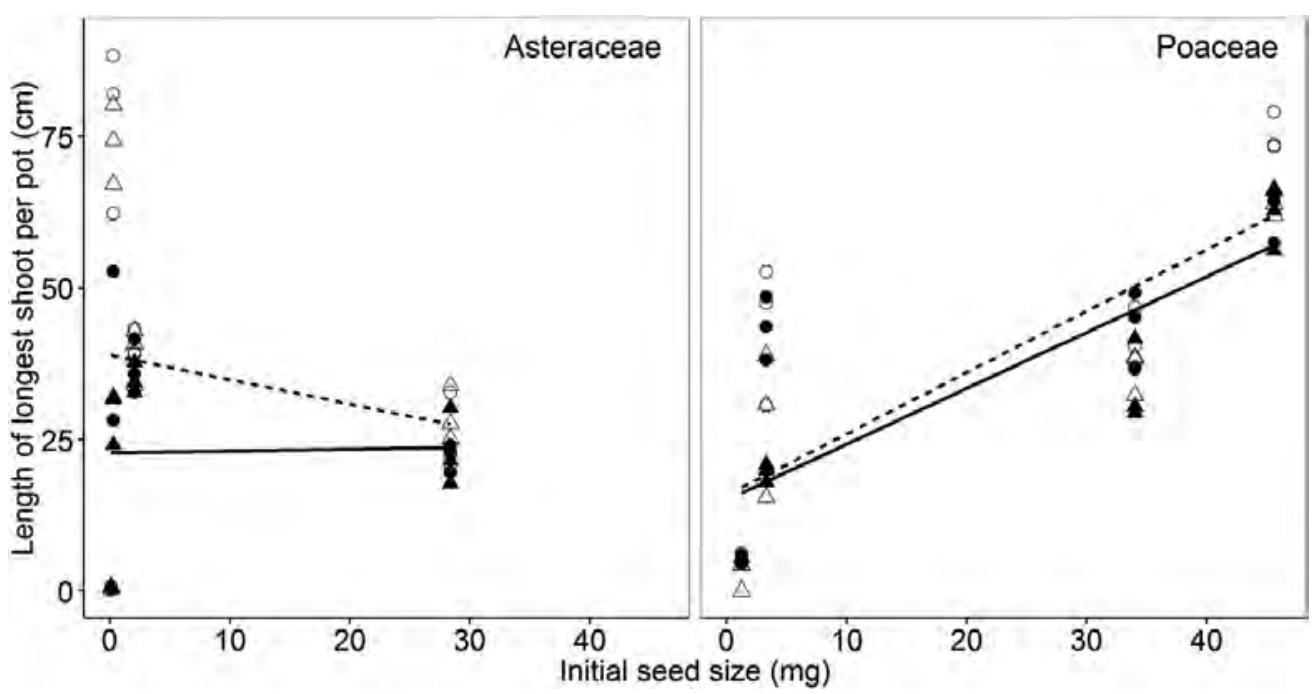

Figure 2.3. - Length of longest shoot of non-native plant species. Asteraceae species (left panel) and Poaceae species (right panel) showed opposing effects of initial seed sizes on the lengths of the longest shoot. The length of the longest shoot was smaller at high (solid symbols, solid line) than at low (open symbols, dashed line) planting densities of native species. The length of the longest shoot did not significantly differ with herbivory (triangles) and without herbivory (circles). Each species is represented by its average initial seed size illustrated here by a vertical stack of points each. Lines represent significant linear regressions (see table table 2.2 for complete statistics). 


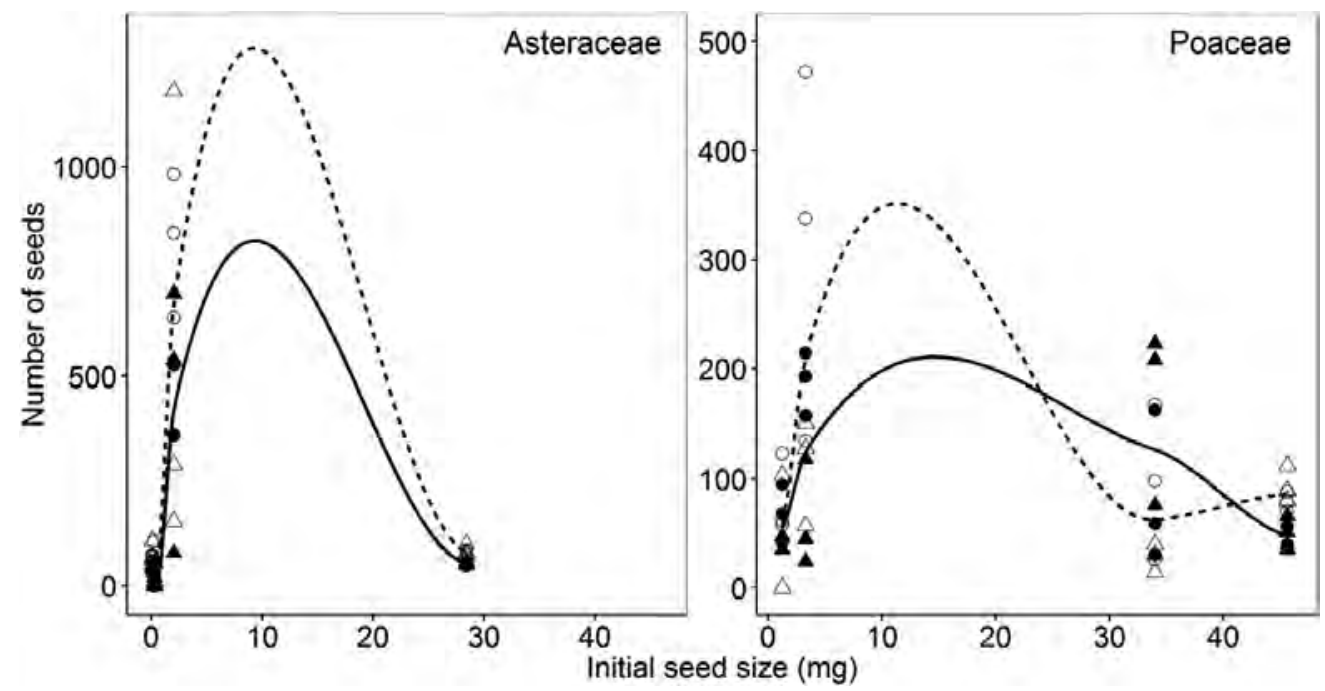

Figure 2.4. - Number of seeds of non-native plant species. Asteraceae species (left panel) and Poaceae species (right panel) showed hump-shaped relationships between numbers of seeds and initial average seed sizes. In general, the curves generated by the smoothing function 'loess' peaked at lower numbers of seeds at high planting density (solid symbols, solid lines) than at low planting density (open symbols, dashed lines) of native species. Under the herbivory treatment (triangles), there tended to be fewer seeds than without herbivory (circles) at the end of the experiment, especially for Poaceae. This indicates a negative effect of granivory and competition on numbers of seeds which is most pronounced at medium initial seed sizes. Each species is represented by its average initial seed size illustrated here by a vertical stack of points each. Note that the scales of $y$-axes differ.

\subsection{Discussion}

Our overall findings indicate a potentially large role of the native plant community in determining the success of non-native plant species. Increased planting density of native species markedly decreased performance parameters of non-native plants, i.e. shoot biomass, shoot length, and fitness in terms of seed number. Interestingly, seed size affected establishment success of non-native species in the opposite manner as expected by our initial hypothesis: Small-seeded species appeared to cope relatively better with high density of native species than large-seeded ones. Note that our use of high and low densities of native species mixtures as proxies of high and low competitive pressure may not necessarily have simulated the maximum competitive effect possible. Instead, our planting densities represented gradual competitive differences.

The decreases in shoot biomass, shoot length, and seed production in the denser native community indicate a pronounced effect of competition on performance of all non-native species. This effect was similar for both families of non-native plants in the experiment. 
In the early establishment phase, individual fitness in terms of seed production will be important for intruding annual plant species, because it can influence population persistence and may ultimately co-determine the rate and extent of range shifts (Jackson and Sax, 2010; Kinlan and Hastings, 2005). Indeed, our results showed that seed production responded less negatively than shoot biomass to competitive pressure of native plants (table 2.3. This may be a result of past selection towards seed production in the face of high native plant densities. Only in the case of $C$. sancta, we observed complete inhibition of seed production in the densest native communities. Assuming that high density is a proxy of the intensity of competition, this finding is in line with the recent suggestion that competition does not necessarily select for high plant biomass, but rather for reproductive ability (Tracey and Aarssen, 2014).

An interesting finding was that large-seeded non-native species may be inhibited more in high-density communities than smaller-seeded species. This is in line with the view that whether the effect of a native community on invaders is negative (biotic resistance) or positive (facilitation) does not only depend on biotic pressures exerted by native species, but also on life history traits of the invading species (Gross et al., 2013) and thus also on the habitat that influenced the evolution of these traits in the original range (Hejda et al., 2015). The seeds in our experiments originated from the same region so that they may be adapted to similar environmental conditions. Although this may limit our insight into genetic and phenotypic variation within single species and differences in evolutionary pressures along latitudinal gradients, we can provide a snapshot of how species from a given community might differ in success of establishing satellite populations beyond their current habitat. Here, we show that life-history traits that are usually related to competitive dominance do not necessarily confer high biomass production and reproductive output during early establishment of non-native species under simulated competitive pressure from a native plant community. Our hypothesis that competitive pressure from native neighbours will have a stronger negative impact on smaller non-native species was not confirmed. One possible explanation lies in the difference between competitive effect and response ability (Goldberg, 1996). The ability to displace neighbours is determined by the rate at which a competitor acquires resources, which likely correlates with seed size. This effect is the basis of size-symmetric competition approaches to explain mortality and size-inequality patterns in plant communities (Schwinning, 1996; Schwinning and Weiner, 1998). However, the 
ability to withstand and tolerate competition could include more traits than size alone. Our findings indicate that enhanced competitive response is associated with small, rather than large seed size. This may indicate that being small could be an advantage in resource competition as resource requirements are allometrically related to biomass (Peters, 1986; Schmitz, 2000). Smaller plants need fewer resources than larger plants and thus are less affected by resource capture of neighbours.

Herbivory did not have a significant impact on plant performance, except for the decrease of seed numbers of grasses due to granivory. These unexpected results reject our original hypothesis and differ from the prevailing view that herbivory has more influence on performance of exotic species than competition (Gonzales and Arcese, 2008; Heard and Sax, 2013). In our experiment, Asteraceae species showed little herbivore damage and Poaceae species showed very high regrowth capacity, i.e. high tolerance against herbivore damage rather than initial repellence strategies. Thus, tolerance against herbivory can be just as important as initial repellence of herbivores as defence strategy (Fornoni, 2011; Hovick et al., 2012). However, the strong granivory damage to Poaceae indicates that non-native plants may be able to recover from herbivory by regrowth within a generation, but the negative impact of herbivores might be postponed to the follow-up generation in the form of decreased reproductive output.

Plant family affected non-native plant performance Asteraceae species generally showed higher values of all response variables than Poaceae, and Asteraceae also tended to show stronger (albeit non-significant) responses to the competition treatment. While grasses are characterized by long, upright leaves, $C$. sancta and $F$. palaestina place their leaves as rosettes on the soil surface. It is likely that for the Asteraceae species the shading effect of the surrounding community was higher than for grasses. Light as a resource can be pre-empted by plants through overtopping smaller plants, giving the larger or more erect plants a strong competitive advantage (Connolly and Wayne, 1996). Thus, plant architecture might hamper performance of invading plants originating from ecoregions with higher light availability. Plant architecture and other traits that differ between and within species of different families should be included in future studies to maybe augment the amount of explained variance. These studies should also include field experiments to avoid any potential pot size limitations that may be particularly relevant for traits such as plant 
height and plant architecture.

Shoot length showed a much smaller reduction at high native plant density than shoot biomass. Moreover, shoot length did not change from small to large seed sizes of Asteraceae, but increased for Poaceae. This may be due to species-specific plant morphology limiting the response of shoot length to increased planting density and seed size to a stronger degree than in the case of shoot biomass. This indicates that biomass may be a more sensitive proxy of density and seed size effects in investigations of non-native plant species establishment.

Survival of non-native plant species was not affected by planting density of natives, while biomass and fecundity was markedly reduced under high density of the natives. Survival of individuals clearly is an essential early step of a successful range expansion. However, reduction of potential offspring through reduction of seed number may impair long-term establishment success, i.e. naturalization, as compared to such species with a lower reduction of potential offspring (Richardson and Pyšek, 2012). Our findings of differential response to neighbours during different life stages is compatible with previous studies, one of which was even conducted within the source communities of our non-native species (Schiffers and Tielboerger, 2006). It has been shown that plant-plant-interactions may shift from positive to negative through the life cycle of plants, i.e. survival may be rather positively affected by neighbours while growth and reproduction are more likely to be inhibited by negative interactions such as competition (Ariza and Tielbörger, 2012; Schiffers and Tielboerger, 2006). In the field, mortality of invading plant individuals may be reduced in the presence of neighbours when these provide shelter from hazards (Kuijper et al., 2004; Ryser, 1993). Therefore, although density-dependent mortality is ubiquitous in plants (Silvertown and Charlesworth, 2001), facilitative interactions may actually play a role in early establishment.

It may be interesting to estimate the net effect through the life cycle of a plant and test whether these positive effects outweigh or balance the negative effects imposed on individual fecundity. Individual fecundity plays out in subsequent generations, when decreased seed numbers lead to lower offspring numbers and vice versa. In our experiment, individual fecundity in terms of seed number showed a right-skewed hump-shaped relationship with initial seed size. Highest reproductive output in the next generation was thus produced by 


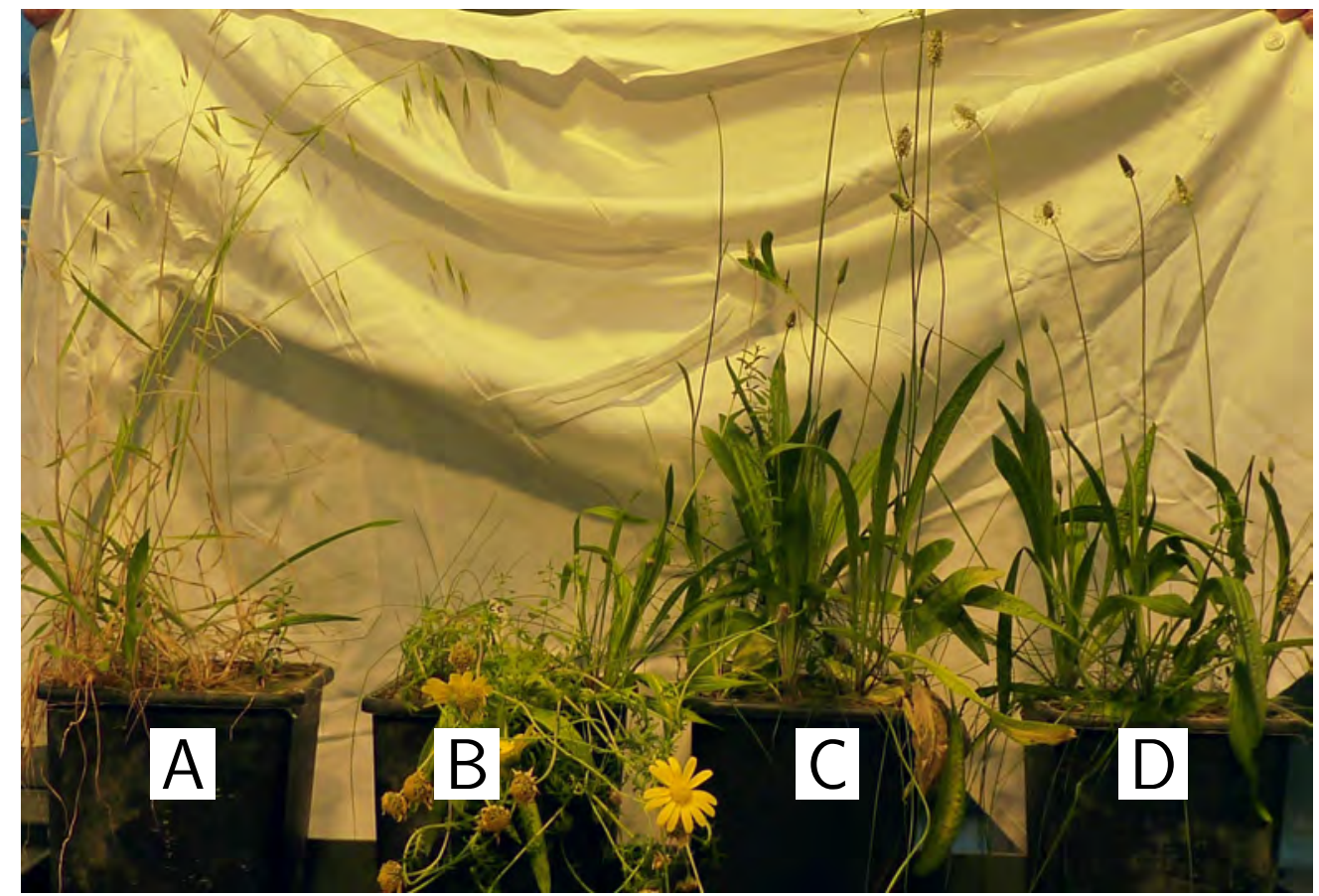

Figure 2.5. - Illustration of dominance of large non-natives: in pots $A$ (A. sterilis) and $B$ (C. coronarium), non-native species dominate the native community and are most prominent in the picture. In pots $C$ (C. sancta) and $D$ (B. fasciculatus), the most prominent individuals are native $P$. lanceolata.

plants with small to medium initial seed sizes. Therefore, plants with small to mediumsized seeds carry a high risk of becoming invasive, since a high number of recruits can override biotic and abiotic constraints and thus enhance invasion speed and extent (Holle and Simberloff, 2005; Warren et al., 2012). In our case, such a positive feedback may be slowed down by competition at the adult stage.

\subsection{Conclusions}

We conclude that in our simulated communities small seed size and small plant size did not generally hamper establishment of non-native plant species in a novel community. Being less susceptible to negative impacts of competition could be the key to successful establishment of non-native species during range expansion. We suggest that more research efforts should go into separating between competitive effect and response and their relationship to seed size with respect to the establishment success of non-native range-expanding plant species in novel communities. Future experiments should also investigate interactions be- 
Performance of non-native annual plant species in a novel community

tween seed size and environmental factors and availability of safe sites for germination, because seed size may not be the overriding factor determining successful establishment. These experiments may further include disturbance treatments to pinpoint the role of disturbance during range expansion, especially were ruderal species are involved as in our case. Extending our experimental design to further community and environment configurations will help to establish the generality of our results for a wider array of range-expanding plant species. 


\title{
3. The role of biotic factors during plant establishment in novel communities assessed with an agent-based simulation model
}

\begin{abstract}
This chapter was published under the same title as pre-print and subsequently as peerreviewed version in PeerJ, August 2018.

Full author list: J Radny , KM Meyer

Declaration of contribution:

$J R$ conceived and designed the experiments, performed the experiments, analyzed the data, prepared figures and/or tables, authored or reviewed drafts of the paper, approved the final draft, developed model concept and code. KM conceived and designed the experiments, analyzed the data, contributed reagents/materials/analysis tools, prepared figures and/or tables, authored or reviewed drafts of the paper, approved the final draft, developed model and concept, reviewed model code, conducted and analyized the sensitivity analysis.
\end{abstract}

Figures 3.1, 3.2 and 3.4 to 3.9 are similar to those published. Figure 3.10 was originally published as supplemental information. Figure 3.3 was not part of the original publication.

The model code was provided as supplementary material with the open access publication.

\begin{abstract}
Establishment success of non-native species is not only influenced by environmental conditions, but also by interactions with local competitors and enemies. The magnitude of these
\end{abstract}


biotic interactions is mediated by species traits that reflect competitive strength or defence mechanisms. Our aim was to investigate the importance of species traits for successful establishment of non-native species in a native community exhibiting biotic resistance in the form of competition and herbivory. We developed a trait-based, individual-based simulation model tracking the survival of non-native plants in a native community. In the model, non-native plants are characterized by high or low values of competition and defence traits. Model scenarios included variation of initial number of non-natives, intensity of competitive interaction, density of herbivores and density as well as mixture of the native community. Traits related to competition had a much greater impact on survival of non-native species than traits related to defence. Survival rates of strong competitors never fell below 50\% while survival of weak competitors averaged at about $10 \%$. Weak competitors were also much more susceptible to competitive pressures such as community density, composition and competition intensity. Strong competitors responded negatively to changes in competition intensity, but hardly to composition or density of the native community. High initial numbers of non-native individuals decreased survival rate of strong competitors, but increased the survival rate of weak competitors. Survival under herbivore attack was only slightly higher for plants with high defensive ability than for those with low defensive ability. Surprisingly, though, herbivory increased survival of species classified as weak competitors. High survival rates of strong non-native competitors relate to a higher probability of successful establishment than for weak competitors. However, the reduced survival of strong competitors at high initial numbers indicates a self-thinning effect, probably mediated by a strongly competitive milieu. For weak competitors, our model emphasizes positive effects of high propagule pressure known from field studies. General effects of herbivory or defence abilities on survival were not supported by our model. However, the positive effect of herbivory on survival of weak competitors indicated side effects of herbivory, such as weakening resident competitors. This might play an important role for establishment of non-natives in a new community.

\subsection{Introduction}

In response to current climate change, range borders and distribution patterns of many species have shifted along with changes in environmental conditions (Chen et al., 2011; 
Parmesan and Yohe, 2003; Walther et al., 2002). The abiotic and biotic environment in novel habitats plays an important role for capturing and eventually predicting range shift dynamics of species (Berg et al., 2010). On a large scale range shifts are correlated with bioclimatic and environmental factors and whether a plant species is able to reach a novel habitat. Thus, range expansion of a species is a continuous process, acting on a large-scale landscape. Realization of range expansion is determined by a series of successful local establishment events of individuals, that build up viable populations in the novel habitat.

Local establishment success strongly depends on biotic interactions with the resident community. In fact, diverging trends in speed, extent and directions of species range shifts may be not only an expression of their ability to reach a novel habitat, but also to persist in novel communities (Lenoir et al., 2010). Such interactions include for example competition with resident plants or herbivore attack (Levine et al., 2004). Local negative processes can add up and might eventually prevent further range shifts. Thus, although a species range is typically described on large, often continental scales, it is limited by small-scale processes at the borders of the range. This is in line with the claim to consider dispersal and local establishment success of species and individuals in order to gain insights on range dynamics (Guisan and Thuiller, 2005; Pearson and Dawson, 2003; Wisz et al., 2013). In a novel habitat, the non-native species will face novel interactions with the resident community. The magnitude of the effect of these interactions is partially determined by the ability of the novel species to respond to the biotic pressures (Kempel et al., 2013; Wisz et al., 2013; ?). This ability is mediated by plant traits. Despite the general acknowledgement of the importance of traits and biotic pressures for establishment success, the interaction of these factors is not well studied in the context of range expansion.

We constructed an individual-based simulation model to scrutinize how these factors together affect success or failure of plant establishment in a novel community. Field surveys are generally incapable of capturing failed invasion attempts (Zenni and Nunez, 2013), but modelling allows for comprehensive systematic modifications and full control of the setup. Data from a preceding greenhouse experiment (Radny et al., 2018) were used as a basis for model construction and parametrization (see chapter 2).

Early establishment of populations in novel habitats in a local context is an essential first step in range shift dynamics of a species. Therefore, in this model we focus on such biotic 


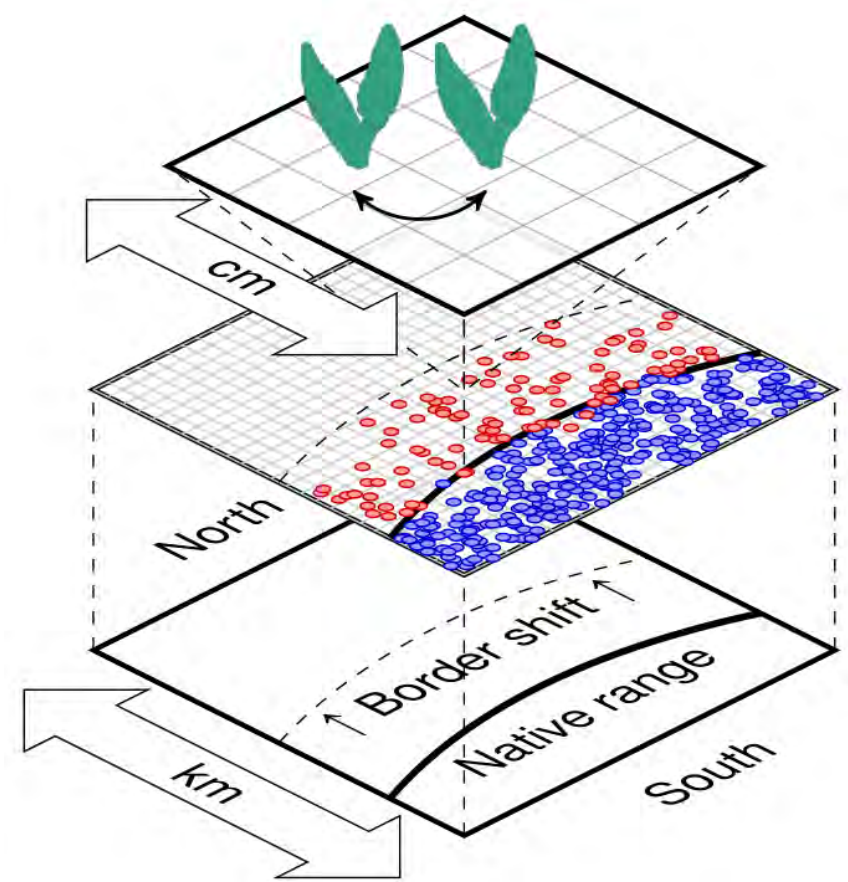

Figure 3.1. - Schematic overview of the scale-dependent relationship between range shift and local establishment of plants. Range shift occurs on a large scale and at species level (A). This movement can be disaggregated into successful establishment events of local satellite populations beyond the native range border (red dots, B). Local persistence depends on the fitness of individuals in this local population. This is influenced by direct interactions, e.g., with neighbouring competitors (plant symbols) or resident herbivores (not shown), on this local scale $(C)$.

interactions that operate on local scales and with immediate contact between the interacting entities. These biotic interactions are enemy attack and competition for resources between neighbouring plants.

Biotic pressures such as competition and enemy attack may hamper establishment success of a novel species in a community by decreasing individual fitness. The causes are obvious: when mutually contested resources are captured by competing species, the inferior competitor can use less of the contested resource for its own growth and reproduction. Eventually, it may die when resource uptake falls below a metabolic threshold (Lin et al., 2012; Schmitz, 2000). Enemy attack can strongly weaken a plant through damage of plant tissue or organs and even result in lethal consumption. Loss of tissue may pose an additive negative effect on plants under competition (Heard and Sax, 2013; Kim et al., 2013; Kuijper et al., 2004). In fact, invasion biology studies have often pointed out that very effectively expanding, i.e., invasive, non-native species suffer less from enemy attack than native or 
non-invasive non-native species (Cappuccino and Carpenter 2005; Matter et al. 2012; but see Heard and Sax 2013.) This effect, referred to as enemy release, is one line of argument to explain invasiveness of non-native plant species in intercontinental invasions (Joshi and Vrieling, 2005; Keane and Crawley, 2002). Since lately enemy release is also studied in intracontinental range shifts, motivated by long-distance dispersal of plant species (Engelkes et al., 2008; Harvey and Fortuna, 2012; Nehrbass et al., 2006).

Traits of species mediate interactions and are often used to describe community composition in classical community ecology (McGill et al., 2006). Intercontinental invasion biology relies on certain traits that are shared by successful invaders to inform risk assessments. However, the trait set of the "perfect invader" remains to be found (Speek et al., 2011). Further, it is largely unclear how strongly trait sets vary under different interaction regimes. For our model, we use the framework of biotic resistance composed of resource competition and herbivore attack (Levine et al., 2004). We describe novel species by a set of traits to respond to these components of biotic resistance. Our central model aim is to assess how non-native species with different trait profiles can establish in a novel community despite negative biotic interactions.

The traits used in the model reflect the ability of a plant to (a) compete with neighbours and (b) defend against enemies. Based on size-symmetric competition models (Connolly and Wayne, 1996; Damgaard and Weiner, 2008), we use seed mass as a measure of plant size and as the trait representing competitive ability (Metz et al., 1992). Other traits have also been associated with competitive strength (Goldberg, 1996), but plant size is a straightforward measure (Domingos, 1999), which is well supported (Aikio, 2004; Weiner and Damgaard, 2006). Mechanical barriers and toxins belong to the traits a plant can use to prevent herbivore attack, and often plants build up a complex defence syndrome of multiple traits (Agrawal and Fishbein, 2006). For our purpose, the form of defence mechanism is not of interest. Thus, we implemented the defence mechanism as the stochastic ability of enemy repulsion.

In summary, the aim of the model presented here is to systematically investigate how local establishment success of non-native species is influenced by biotic resistance of the native community, by competition and defence traits of non-natives, and by the interaction of biotic resistance and traits. We present a comprehensive analysis of the consequences of a 
Table 3.1. - Parameter values for traits of non-native plants. Seed weight was directly measured and averaged from plant material used in the preceding experiment (Radny et al., 2018) and based on the species $S$. capensis and $B$. fasciculatus. We chose maximum biomass relative to the native community. Repulsion of herbivores was averaged over all strongly and weakly defended species in the preceding experiment respectively.

\begin{tabular}{llll}
\hline Parameter & Unit & \multicolumn{2}{c}{ Value } \\
\hline Competitive strength values & & Strong competitors & Weak competitors \\
Seed weight & $\mathrm{mg}$ & 3 & 1 \\
Maximum biomass & $\mathrm{mg}$ & 15.000 & 4.000 \\
Defensive strength values & & Strong defenders & Weak defenders \\
Repulsion of herbivores & {[]} & 0.7 & 0.3 \\
\hline
\end{tabular}

broad range of parameters representing biotic resistance and species traits for non-native species survival and thus local establishment.

\subsection{Model description}

Our model description follows the ODD protocol (Grimm et al., 2006, 2010). Model structure is partly based on (Lin et al., 2012).

\subsubsection{Purpose}

The model compares first generation establishment success of generic range expanding species in a novel community, based on a hypothetical ecological profile. The ecological profile is expressed by combinations of high or low ability to compete with neighbouring plants and defend against insect herbivores. We assign seed size to competitive ability and repulsion of herbivores to defence ability (table 3.1). In the following, generic species are called "species" for better readability.

\subsubsection{Entities, state variables, and scales}

We implemented two general types of agents, plants and herbivores. Plants are non-mobile agents, characterized by their $\mathrm{x}$-y-position and values related to growth, reproduction and defence (tables 3.1 and 3.2). On initialization, the plants are represented as seeds and hatch in the first step. We distinguish two general types of plants, native and non-native, 
Table 3.2. - Parameter values for traits of native plants. Note that maximum age is aligned for all species. Reproduction and dispersal values are not considered for native species as we were not interested in the fate of native species in this approach. Parameter values were either directly retrieved or approximated $\left({ }^{*}\right)$ from the available literature. The composition of the native community in uneven mixtures is based on seed bank values. Initial seed density for $F$. rubra $\left({ }^{* *}\right)$ has been approximated from vegetation cover (30 percent) as retrieved from Edwards and Crawley (1999), assuming that all seeds germinate and build the entire native community.

\begin{tabular}{|c|c|c|c|c|}
\hline Species & Parameter & Unit & Value & Source \\
\hline \multirow{4}{*}{ Plantago lanceolata } & Seed weight & $\mathrm{mg}$ & 1.4 & Schmitt et al. (1992)* \\
\hline & Maximum biomass & $\mathrm{mg}$ & 8.000 & Janeček et al. (2014) \\
\hline & Repulsion of herbivores & [] & 0.4 & Radny et al. (2018) \\
\hline & Initial seeds & $\frac{1}{m^{2}}$ & 85 & Edwards and Crawley (1999) \\
\hline \multirow{4}{*}{ Hypericum perforatum } & Seed weight & $\mathrm{mg}$ & 0.09 & Fox et al. (1999) \\
\hline & Maximum biomass & $\mathrm{mg}$ & 5.800 & Vilà and Weiner (2004) \\
\hline & Repulsion of herbivores & [] & 0.9 & Radny et al. (2018) \\
\hline & Initial seeds & $\frac{1}{m^{2}}$ & 98 & Matus et al. (2005) \\
\hline \multirow{4}{*}{ Agrostis capilaris } & Seed weight & $\mathrm{mg}$ & 0.06 & Stukonis and Slepetys (2013) \\
\hline & Maximum biomass & $\mathrm{mg}$ & 4.000 & Ehlers (2011) \\
\hline & Repulsion of herbivores & [] & 0.3 & Radny et al. (2018) \\
\hline & Initial seeds & $\frac{1}{m^{2}}$ & 1.343 & Edwards and Crawley (1999)* \\
\hline \multirow{4}{*}{ Festuca rubra } & Seed weight & $\mathrm{mg}$ & 0.77 & Larson et al. (2001) \\
\hline & Maximum biomass & $\mathrm{mg}$ & 12.000 & Corbin and D'Antonio (2004)* \\
\hline & Repulsion of herbivores & [] & 0.3 & Radny et al. (2018) \\
\hline & Initial seeds & $\frac{1}{m^{2}}$ & 654 & Edwards and Crawley (1999)** \\
\hline
\end{tabular}

and twelve species, of which four are native and eight are non-native. Parameters have been retrieved from a preceding experiment (Radny et al., 2018) and from the literature.

The simulated world comprises of a homogenous $100 \times 100$ cell grid. Each grid cell represents $1 \mathrm{~cm}^{2}$, thus we model a $1 \times 1 \mathrm{~m}$ plot. To avoid edge effects, the world is a torus, i.e., opposing edges are connected Grimm and Railsback (2005).

Each time step represents one day. A simulation comprises 76 days, i.e., the average lifespan of a non-native species in the preceding experiment. The simulation was aborted earlier if no non-native individuals were left on the grid.

\subsubsection{Process overview and scheduling}

During each time step, the simulation routine is exerted in the following order: herbivores appear and feed on random plants, competition intensity is calculated for each plant individual, plants grow according to this competition intensity and plants age. After 76 days, 
the plants produce and disperse seeds and die. Dead plants are removed from the grid.

The process schedule is visualized in fig. 3.2.

\subsubsection{Basic principles}

We follow the assumption that community composition can at least partly be related to biotic interactions and that biotic interactions are closely connected to functional traits of species (McGill et al., 2006). We apply a basic profile of functional traits related to the ability of species to (a) compete with neighbours and (b) defend against enemies. We also address biotic resistance, i.e., negative impacts of resident herbivores and competitors on non-native plants, which can influence local establishment success (Kempel et al., 2013).

\subsubsection{Emergence}

Several patterns related to individual plant devel-

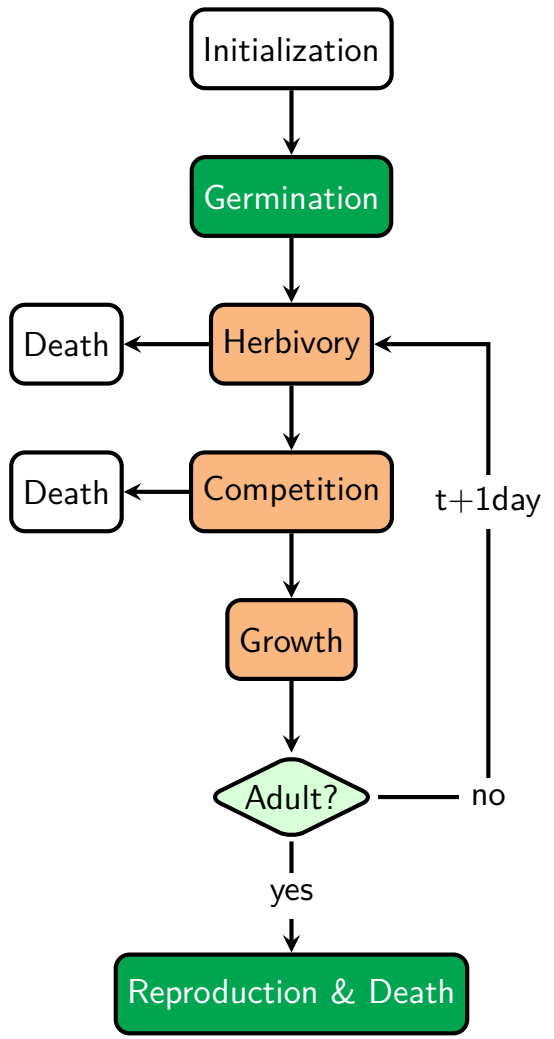
opment and population dynamics emerge from the model. Examples include spatial patterns of plant

Figure 3.2. - Order of executed routines during each simulation. Greencoloured routines are executed only individuals, population dynamics of non-native and once; orange-coloured routines are executed on a "daily" basis, i.e., at native plants, reproductive output, and plant size distributions. For the purpose of this paper, we foeach time step. Herbivory and Competition can lead to individual death of plants.

cus on initial and final number of individuals of nonnative species per time step as a basis for deriving survival rate in the establishment phase (see section 3.2.12).

\subsubsection{Interactions}

We explicitly model competitive interactions between neighbouring plants via the ZoneOf-Influence (ZOI) approach (Lin et al., 2012; Weiner and Damgaard, 2006). Furthermore, herbivores interact with plants by consuming parts of the aboveground biomass of the 
encountered plants.

\subsubsection{Stochasticity}

Plant individuals are initialized with random $x$-y-coordinates. To reflect individual deviance from population mean values, initial biomass of plants is calculated as seed mass multiplied by a random number, drawn from a normal distribution with a mean of 1 and a standard deviation of 0.1 . Instead of defining fixed relative growth rates ( $r g r)$, plants determine their own growth rate through

$$
\frac{\ln (\text { maximum biomass })-\ln (\text { initial biomass })}{\text { maximum age }}
$$

(Hunt, 1982). This approach propagates the stochasticity involved in initial biomasses to the distribution of relative growth rates. Daily density of herbivores is stochastic, as grasshopper density is multiplied with a stochastic number of meals per herbivore (see Submodels: Herbivory). Success of each foraging attempt of herbivores on a given plant is stochastic, based on the repulsion value of the plant.

\subsubsection{Initialization}

Each simulation run starts with placement of seeds on the grid at random xy-positions. Initial native community population size is based on plant densities in the preceding greenhouse experiment (Radny et al., 2018). In that experiment, planting pots of $18 \times 18 \mathrm{~cm}$ carried either 12 or 44 native individuals, i.e., $\sim 300$ or 1,100 individuals per $\mathrm{m}^{2}$. Mixtures of native species are based on either the experiment or approximated literature values (table 3.2) depending on the simulation scenario (table 3.3). For experiment-based mixtures, each native species is represented with the same number of individuals, called "even mixture" hereafter. For literature values, we use the reported seed bank size of the respective species as initial numbers (table 3.2), called "seedbank mixture" hereafter. Per simulation scenario, there is one non-native species mixed into the native community. Different simulation scenarios were run with initially 8, 16, 32, 64, 128 and 256 seeds of non-native species. These scenarios reflect different levels of introduction efforts or proximity to core 
range with higher population densities (Kolar and Lodge, 2001).

\subsubsection{Input data}

The model does not require any input data beyond the model parameters.

\subsubsection{Submodels}

\section{Herbivory}

Herbivore agents are created at the beginning of each time step (fig. 3.2). The grasshopper Locusta migratoria is used as herbivore model species due to its wide distribution ( $C A B I$, 2013) and generalist feeding behaviour (Macel et al., 2005; Schmitz, 1997). We had also chosen this species as herbivore in the preceding experiment (Radny et al., 2018).

Herbivore density is varied based on literature values and density in the experiment, ranging from 3.2 grasshoppers per $\mathrm{m}^{2}$ (literature value Ledergerber et al. 1997) to 75 grasshoppers per $\mathrm{m}^{2}$ (experimental value, based on Morriën et al. 2011). One scenario is implemented without herbivores (table 3.3). Here, neither native nor non-native species experience herbivory.

L. migratoria consumes 50-70 mg of plant material per day Simpson and Abisgold (1985). Consumption does not happen all at once, but in 7 to 10 meals of about $7.5 \mathrm{mg}$ each. In a natural setting, it is not likely that a highly mobile insect is feeding on the same plant individual all day. Thus, we implemented in-between meal movement. To reduce computing effort, we created one dummy herbivore individual per meal. The number of dummy herbivore individuals that represent one herbivore was determined by multiplying each herbivore by a random number drawn from the interval $[7,10]$. This may, for instance, lead to eight dummy herbivore individuals representing eight meals of herbivore $A$ plus ten dummy herbivore individuals representing ten meals of herbivore $B$ and so forth. The movement of the dummy herbivore individuals reflects in-between meal movement of the original herbivores. This simplification is justified, because we are not interested in the fate of individual herbivores, but just their effect on plant biomass. This multiplication allows us to model a process which is operating at a finer temporal scale than the global time step 
(one day).

Each dummy herbivore is initialized at random $x-y$-coordinates and approaches a random plant in a radius of 50 patches. To consume the approached plant, a random number drawn from the interval $[0,1]$ must be greater than the repulsion value of the respective plant. $A$ high repulsion value thus represents high defence of a plant, e.g., through physical barriers or toxins. If dummy herbivores are not successful with foraging, they leave that meal out and are removed from the grid. This procedure still adequately represents the overall effect of herbivory on plants. All successful dummy herbivores are removed directly after foraging. Thereby, herbivore density fluctuates daily. As we observed in our greenhouse experiment (Radny et al., 2018), a plant dies in the model if more than $90 \%$ of its current biomass is consumed by herbivores, and it is removed from the grid.

\section{Plant competition and growth}

Plants interact competitively with their neighbours. To model competition, we follow the Zone-Of-Influence (ZOI) approach (Berger et al., 2008; Lin et al., 2012; Weiner and Damgaard, 2006; Weiner et al., 2001). The ZOI of a plant is represented by a circular area $A_{Z O I}$ with radius $r$. Based on Lin et al. (2012), radius $r_{i}$ is allometrically related to biomass $B$ of plant $i$ at time $t$ :

$$
r_{i}=B_{i}(t)^{3 / 8} * \sqrt{\frac{1}{\pi}}
$$

Note that $B(t)$ is determined after herbivore attack. Based on Lin et al. (2012), plant growth in the next time step is determined by the relative plant growth rate $\mathrm{rgr}$, the area of the $\mathrm{ZOI} A_{Z O I}$, current biomass $B$ and maximum biomass $B_{\max }$ :

$$
\frac{d B}{d t}=r g r * A_{Z O I} *\left(1-\left(\frac{B}{B_{\max }}\right)^{1 / 4}\right)
$$

In most cases, the $\mathrm{ZOI}$ of a plant covers several grid cells. Thus, $A_{Z O I}$ consists of the sum of the area of the grid cells $c$ that are occupied. Equation 3.3 applies when a plant is 


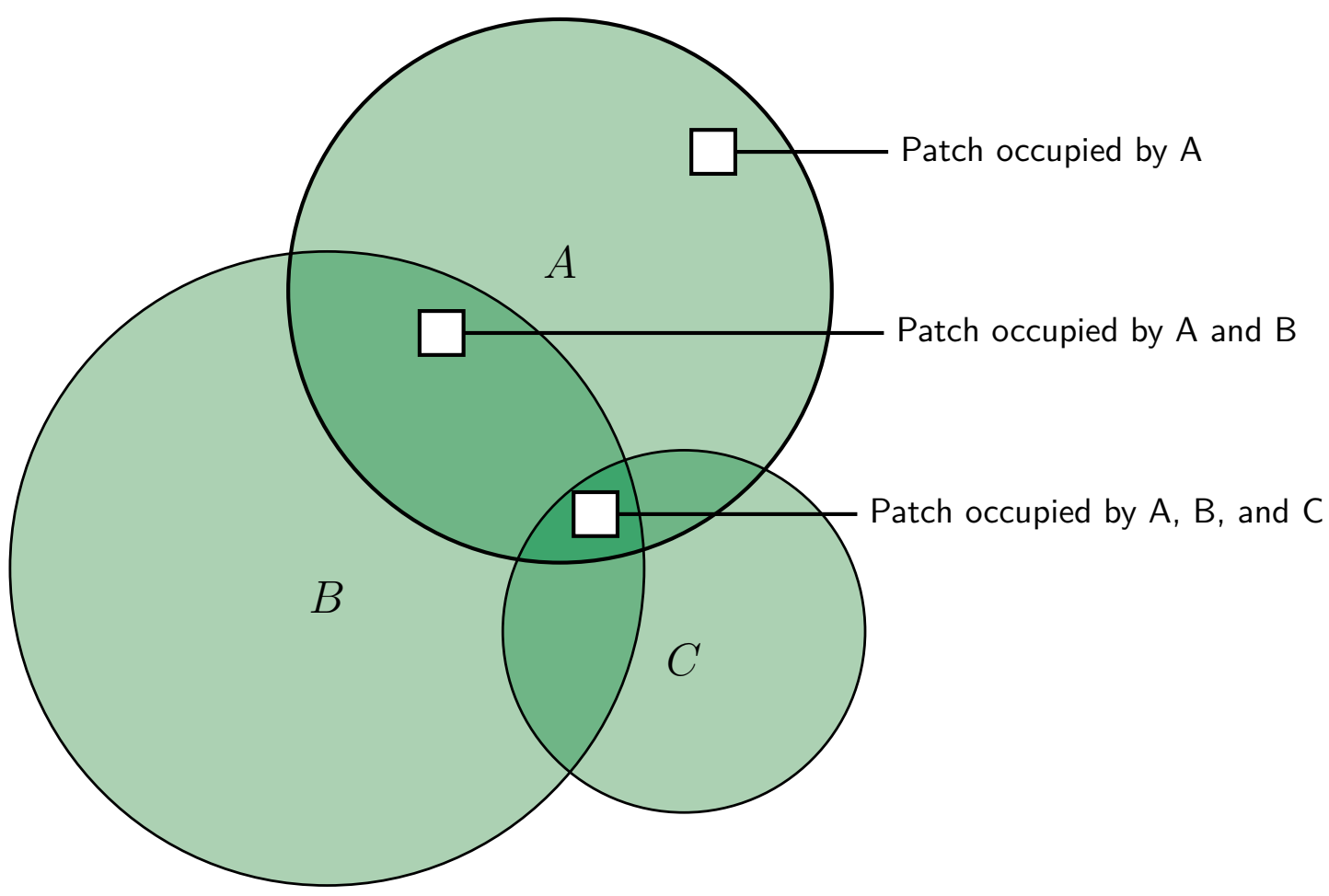

Figure 3.3. - Illustration of the Zone-of-Influence (ZOI) concept with three plant individuals. Each plant is represented as green circle with radius $r$ related to biomass of the plant (see eq. 3.2). Each plants' ZOI comprises several grid cells. Patches in light green areas are occpuied by one plant only. Ressources on these patches are captured by that plant alone as described in eq 3.3. Where ZOls of plants overlap, the individual plants share the available resources according to the degree of asymmetry of competition (see eq. 3.5)

growing without neighbours, i.e., none of the cells within $A_{Z O I}$ is occupied by another but the focal plant.

When two or more plants are neighbours, i.e., their ZOls overlap in at least one cell (fig. 3.3), plant $i$ calculates its effective area $A_{e f f ; i}$ as the sum of the area of the grid cells $c$ it occupies, each weighted by the proportion of its current biomass $B_{i}(t)$ relative to the total biomass of all overlapping plant individuals $j$ to $k$ in this grid cell, modified by the degree of asymmetry of competition $\Theta$ (Lin et al., 2012; Weiner and Damgaard, 2006):

$$
A_{e f f, i}(t)=\sum_{c_{i}} \frac{B_{i}(t)^{\Theta}}{\sum_{i}^{k} B_{j}(t)^{\Theta}} d c
$$

where $d c$ is the area of the respective grid cell $c$. In our case, all grid cells have the same area. Thus, instead of using $d c$ as in eq. (3.4), the summed area of the grid cells of the 
ZOI of plant $i$ can be rearranged and expressed in terms of the biomass of plant $i$ (Lin et al., 2012):

$$
A_{e f f, i}(t)=B_{i}(t)^{3 / 4} \sum_{c_{i}} \frac{B_{i}(t)^{\Theta}}{\sum_{i}^{k} B_{j}(t)^{\Theta}}
$$

We use the degree of asymmetry as a measure of competition intensity. Intensity of competition determines how the contested resources at any patch are shared among the competing plants, depending on their biomass relative to the other competitors. With $\Theta=0$, resources are shared equally among plants regardless of their biomass. Increasing $\Theta$ leads to an increasing weight of biomass for capturing contested resources in a shared cell. With $\Theta 1$, a larger plant receives a larger share of the contested resources than a smaller plant, proportional to its biomass (perfect size-symmetry). Mathematically, $\Theta$ can be located between 0 and $\infty$ (Schwinning and Weiner, 1998), but for our purpose, we use $\Theta=0,0.5$ and 1 in different model runs. Plant growth under competition is implemented following Lin et al. (2012) as:

$$
\frac{d B}{d t}=r g r * A_{e f f} *\left(1-\left(\frac{B}{B_{\max }}\right)^{1 / 4}\right)
$$

The difference between eqs. (3.3) and (3.6) is that either all resources within the zones of influence are considered to calculate growth regardless of neighbouring competitors $\left(A_{Z O I}\right)$, or that only the share of resources captured after competition with neighbours are considered to calculate growth $\left(A_{e f f}\right)$. If growth and resource uptake fall below the threshold of 0.05 of $B^{3 / 4}$, the plant cannot serve its metabolic costs and dies (Lin et al., 2012; Schmitz, 2000). Overall, plant growth is strongly determined by plant biomass in our model (fig. 3.10) 


\section{Plant reproduction and dispersal}

When plants reach maturity, i.e., maximum age, they produce seeds as a function of their final biomass. So under strong competition, final biomass does not necessarily match maximum biomass. In our greenhouse experiment (Radny et al., 2018), we obtained a positive correlation between aboveground biomass and seed mass, i.e., total mass of all seeds produced per plant. We use this empirical correlation to determine seed mass per plant in the model, i.e., a ratio of $0.41 \mathrm{mg}$ seed mass per $\mathrm{mg}$ shoot biomass for strong competitors and $0.65 \mathrm{mg}$ seed mass per $\mathrm{mg}$ shoot biomass for weak competitors. The number of seeds per plant is implemented in the model as seed mass produced by the respective plant divided by average weight of a single seed (table 3.2). We included stochastic variation of number of seeds of $\pm 10 \%$. To reduce computational ballast, the number of seeds produced is reduced by winter mortality, including seed predation, and germination probability is applied. Thus, only seeds that survive winter and germinate in the next year are explicitly created. Seeds are then dispersed in a random angle with dispersal distance following a Weibull distribution to allow for long-distance dispersal (Colbach and Sache, 2001; Paradis et al., 2002). Number of seeds remaining on or being dispersed beyond the parental patch is recorded together with dispersal distance of each seed for future scaling-up of the model.

\subsubsection{Model parametrization and validation}

Model parameters were partly derived from literature and partly from a preceding greenhouse experiment (Radny et al., 2018, see also chapter 2). The greenhouse experiment was conducted at the NIOO-KNAW in Wageningen, The Netherlands. There, we planted microcosms of $18 \times 18 \mathrm{~cm}$ with a native community of four plant species mixed with one non-native species per microcosm. The non-native species in the experiment differed in life-history traits related to competitive and defensive strength. In a fully crossed design, non-native species were exposed to two densities of natives to create different intensities of competitive pressures. Low density microcosms were planted with 12 individuals of native species and four individuals of one non-native species. High density microcosms were planted with 44 individuals of the native species and four individuals of one non-native species. Half of the microcosms were exposed to herbivory by two generalist herbivore species, Locusta migratoria (L.) and Mamestra brassicae (L.). We harvested the micro- 
cosms when non-native individuals had finished their seed production and recorded dry aboveground biomass, seed mass, and other performance parameters (Radny et al., 2018).

For parameterization of non-native species in the model, we used data on the Mediterranean grass species Stipa capensis (Thunb.) and Bromus fasciculatus (C.Presl) from our experiment and from the literature (table 3.1). The model differentiates between four trait profiles of generic non-native species: strong versus weak competitors combined with strong versus weak defenders.

Competitive strength is represented by average seed weight of $S$. capensis for strong competitors and B. fasciculatus for weak competitors (table 3.1). Maximum biomass was assigned as a value relative to the native community (table 3.2). Additionally, we derived the fitness measure biomass-seed mass ratio from the preceding experiment by dividing total seed mass by total aboveground biomass of a species. This ratio was different for strong and weak competitors in the experiment and thus serves as another distinctive index for competitive strength (table 3.1).

Defensive strength was modelled as repulsion of herbivores reflecting the chance of a plant to avoid herbivore attack. Repulsion values were derived from the percentage of individuals without or less $(>5 \%)$ clearly visible leaf damage of all individuals of a species that were exposed to herbivory during the preceding experiment. Values for strong defenders were obtained from B. fasciculatus, whereas values for weak defenders were obtained from $S$. capensis (table 3.1).

The parameterization of the native community in the model is based on the species in the experimental native community, i.e., Agrostis capillaris (L.), Festuca rubra (L.), Hypericum perforatum (L.) and Plantago lanceolata (L.). These four species are fairly widespread over Europe (Roscher et al., 2004), and thus range-expanding plants from southern latitudes are likely to encounter these species as interaction partners. With the exception of repulsion of herbivores which was based on the overall observations of high and low attacks in the preceding experiment, parameters used in the model for native species were based on literature data (table 3.2).

No independent data were available for direct comparison with model outputs. Therefore, we discussed comparisons of model outputs with the results of our preceding experiment 


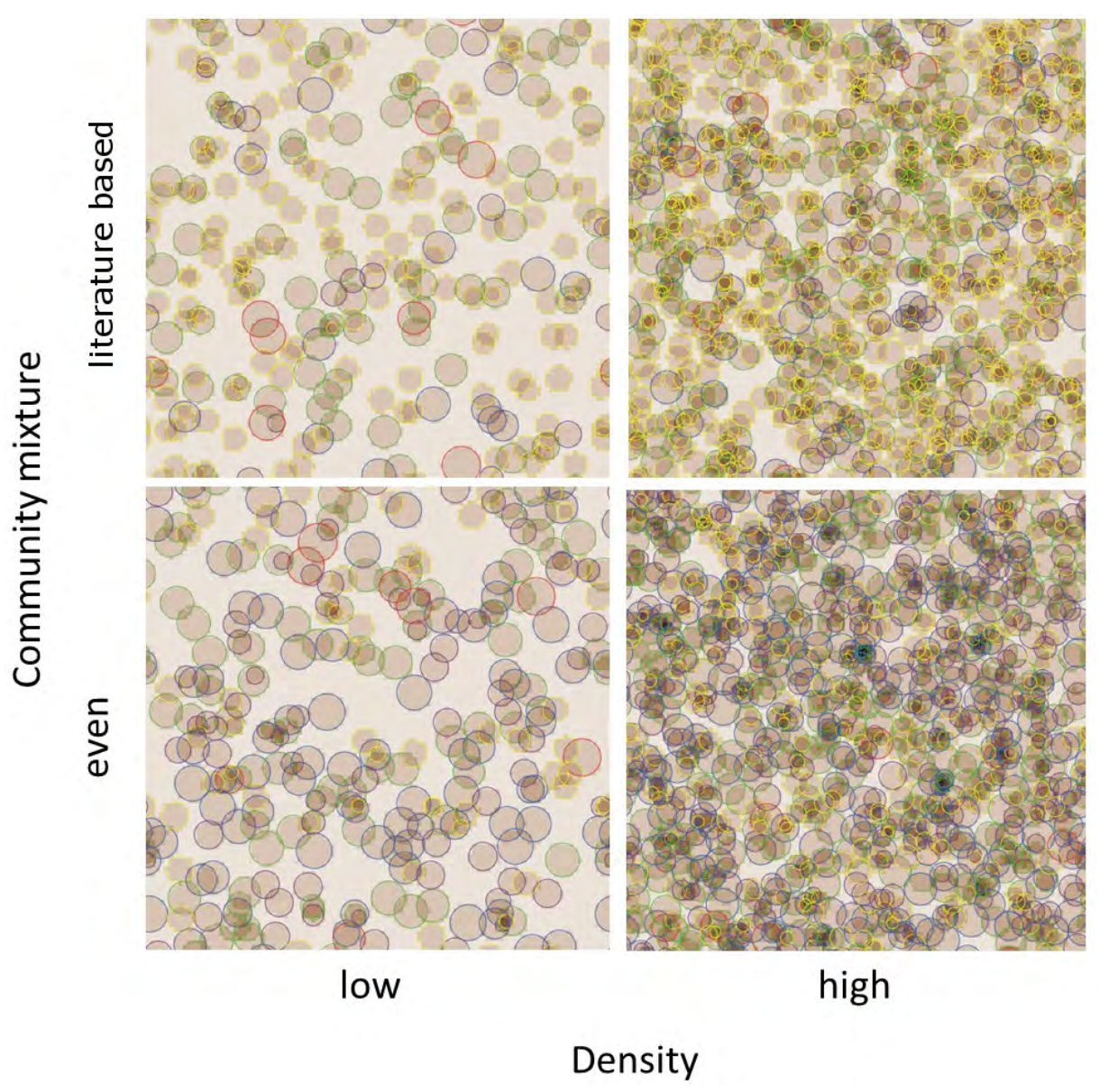

Figure 3.4. - Screenshot of model communities after 14 days. Model community is shown at varying initial community density (low: 308 plants, high: 1,108 plants) and varying mixtures of the native community (even: same initial density of all species; literature based: species initial densities differ based on reported seed bank sizes and germination rates). Species are colorcoded: Red = non-native species, all other colors: native species, i.e., yellow $=A$. capilaris, green $=F$. rubra, purple $=H$. perforatum, blue $=P$. lanceolata.

and conducted a scenario analysis (table 3.3 ) and a sensitivity analysis to systematically explore the parameter space to explain as much output variation as possible.

\subsubsection{Scenario analysis}

The major goal of our simulation model was to investigate the local establishment of nonnative species in a novel community, based on different trait configurations. To assess local establishment, we determined final population size, i.e., the number of reproducing adults at the end of a simulation run.

To account for scenario-based differences in initial population sizes, we calculated survival 
Table 3.3. - Overview of components of model scenarios. Model scenarios were put together by choosing one level per component. All possible combinations of levels were run in our model analysis.

\begin{tabular}{llll}
\hline $\begin{array}{l}\text { Components } \\
\text { model scenario }\end{array}$ & $\begin{array}{l}\text { Number } \\
\text { of levels }\end{array}$ & Levels \\
\hline Trait profiles & 4 & $\begin{array}{l}\text { high competitive and high defensive (HiAll), high } \\
\text { competitive and low defensive (HiComp), low } \\
\text { competitive and high defensive (HiDef), low com- } \\
\text { petitive and defensive (LowAll) }\end{array}$ \\
$\begin{array}{l}\text { Initial population size } \\
\text { of non-natives }\end{array}$ & 6 & $8,16,32,64,128,256$ \\
$\begin{array}{l}\text { Competition intensity } \\
\text { or asymmetry }(\Theta)\end{array}$ & 3 & $0,0.5,1$ \\
$\begin{array}{l}\text { Community density } \\
\text { nerbivore density }\end{array}$ & 2 & $\begin{array}{l}\text { same initial density of all species (even), species } \\
\text { initial densities differ based on reported seed bank } \\
\text { sizes and germination rates (literature based) }\end{array}$ \\
\hline
\end{tabular}

rate as the ratio of final and initial numbers of non-native species. Model scenarios include the four different trait profiles (high and low competitive and defensive strength), six levels of initial population size of non-natives, three levels of competition intensity, two levels of community density, two levels of mixture of the native community, and three levels of herbivore density (table 3.3 and fig. 3.4). The competition index $\Theta$ describes the degree of intensity or asymmetry in competition and takes the values $0,0.5$ and 1 in our model. For the factor mixture of native community, native individuals are either mixed evenly or the percentage of native individuals of a species is derived from literature values about seedbank sizes (for more detail, see section 3.2.8).

To reach our overall goal, we applied a binomial generalized linear model of the form:

Survival of non-natives $\sim$ competitive strength of non-natives + defensive strength of nonnative species + initial population size of non-natives + competition index $\Theta+$ native species density + mixture of native species + herbivore density

and all two-way interactions.

To account for overdispersion, we used a quasibinomial model. We simplified the generalized linear model by removing non-significant terms $(p \leq 0.05)$ one by one, starting with 
the interactions, until only significant terms were left in the model. If a non-significant main term was part of at least one significant interaction term, we kept the main term in the model. This procedure led to the removal of the interactions between native species density and mixture of native species, defensive strength of non-natives and mixture of native species, and competitive and defensive strength of non-native species. We used a chi-squared test to establish that the residual deviance was significantly reduced compared to the residual deviance of the null model. We used the software $\mathrm{R}$ version 3.3.3 (Team, 2016) for data analysis.

\subsubsection{Sensitivity analysis}

We conducted a sensitivity analysis to assess the relative importance of the input parameters with respect to model output. To this end, we used the same combinations of parameter values as in the scenario analysis (table 3.3) except for the parameters mixture of the native community and trait profile of the non-native species. Instead of these parameters, we ran sensitivity simulations with only one native species and one non-native species each, which were called species 1 and species 2 . This approach made it possible to systematically assess the full range of possible trait values and not just the fixed trait profiles used for native and non-native species in the scenario analysis. The tested traits were seed weight, maximum biomass, repulsion of herbivores, seed mass per shoot mass, and germination probability for species 1 and species 2 . To save computing time and still cover as much trait parameter space as possible, we applied Latin Hypercube Sampling (McKay et al., 1979) to assemble the trait profiles for the sensitivity analysis.

We drew 15 samples from uniform distributions in the cases of repulsion (between 0 and 1) and germination probability (between 0.002 and 0.9 ) and from log2-distributions in the cases of seed weight (between 0.06 and 6), maximum biomass (between 3,000 and 30,000), and seed mass per shoot mass (between 0.02 and 6).The sampling distributions and ranges were determined based on our experience with the model simulations and always captured the range of the standard model parameterization. The values for each trait were randomly rearranged and then assembled into 15 trait profiles for species 1 and 15 trait profiles for species 2. Simulations were conducted with the full factorial combination of the remaining parameters from the scenario analysis (table 3.3) for each of the 15 combinations of trait 
profiles of species 1 and 2. Model output was survival of species 2 .

The results of the simulations were analysed with a generalized linear model with quasibinomial errors in the same way as in the scenario analysis. This included model simplification and checking. To assess the sensitivity of the model output to the input parameters, standardized model coefficients were calculated by dividing model estimates by their standard errors. The absolute values of the standardized model coefficients were divided by their sum to obtain sensitivity values between 0 and 1 . Sensitivities were sorted and plotted for comparability.

\subsection{Results}

Table 3.4. - Effects of trait values (competitive and defensive ability) and community configuration (herbivore density, community density and mixture, intensity of competition and initial number of non-natives) on survival of nonnative species in a generalized linear model with quasibinomial errors presented as estimates of the effects and their corresponding standard errors, $t$-values and p-values. The Intercept corresponds to low competitive ability, low defensive ability, high community density and even community mixture. Asterisks indicate p-values smaller than 0.01 $\left({ }^{* *}\right)$ or $0.001\left(^{* * *}\right)$.

\begin{tabular}{lrrrrr}
\hline & Estimate & Std. Error & $\boldsymbol{t}$-value & $\boldsymbol{p}$-value & \\
\hline Intercept & -2.43 & 0.020 & -118.68 & $<0.001$ & $* * *$ \\
High competitive ability (comp) & 10.38 & 0.033 & 312.34 & $<0.001$ & $* * *$ \\
High defensive ability (Def) & -0.22 & 0.017 & -13.05 & $<0.001$ & $* * *$ \\
Initial number of non-natives (InitNN) & 0.006 & 0.000 & 75.87 & $<0.001$ & $* * *$ \\
Herbivore density (DensHerb) & 0.48 & 0.010 & 46.68 & $<0.001$ & $* * *$ \\
Competition intensity $\Theta$ (Theta) & -2.61 & 0.032 & -81.89 & $<0.001$ & $* * *$ \\
Low community density (DensComm) & 0.53 & 0.017 & 31.77 & $<0.001$ & $* * *$ \\
Uneven community mixture (Mix) & -0.005 & 0.016 & -0.34 & 0.73 & \\
Comp:InitNN & -0.014 & 0.000 & -141.82 & $<0.001$ & $* * *$ \\
Comp:DensHerb & -0.28 & 0.008 & -32.81 & $<0.001$ & $* * *$ \\
Comp:Theta & -2.67 & 0.021 & -127.50 & $<0.001$ & $* * *$ \\
Comp:DensComm & -1.20 & 0.014 & -82.55 & $<0.001$ & $* * *$ \\
Comp:Mix & -0.67 & 0.014 & -47.95 & $<0.001$ & $* * *$ \\
Def:InitNN & 0.0005 & 0.000 & 7.59 & $<0.001$ & $* * *$ \\
Def:DensHerb & 0.023 & 0.006 & 3.89 & $<0.001$ & $* * *$ \\
Def:Theta & 0.14 & 0.011 & 12.80 & $<0.001$ & $* * *$ \\
Def:DensComm & 0.064 & 0.010 & 6.63 & $<0.001$ & $* * *$ \\
InitNN:DensHerb & -0.0004 & 0.000 & -11.44 & $<0.001$ & $* * *$ \\
InitNN:Theta & -0.001 & 0.000 & -10.09 & $<0.001$ & $* * *$ \\
InitNN:DensComm & 0.0008 & 0.000 & 12.07 & $<0.001$ & $* * *$ \\
InitNN:Mix & 0.0005 & 0.000 & 7.29 & $<0.001$ & $* * *$ \\
DensHerb:Theta & -0.121 & 0.009 & -12.88 & $<0.001$ & $* * *$ \\
DensHerb:DensComm & -0.158 & 0.006 & -26.61 & $<0.001$ & $* * *$ \\
DensHerb:Mix & -0.016 & 0.006 & -2.71 & $<0.01$ & $* *$ \\
Theta:DensComm & 0.544 & 0.016 & 33.49 & $<0.001$ & $* * *$ \\
Theta:Mix & 0.629 & 0.016 & 40.43 & $<0.001$ & $* * *$ \\
\hline & & & & &
\end{tabular}


All main and interaction terms in the generalized linear model contributed significantly to survival of non-native species $(p<0.01)$ except for mixture of native species (table 3.4$)$. The importance of competitive strength was very dominant and was a much stronger source of variation than defensive ability for survival of non-natives (main effects Comp and Def in table 3.4 and fig. 3.5).

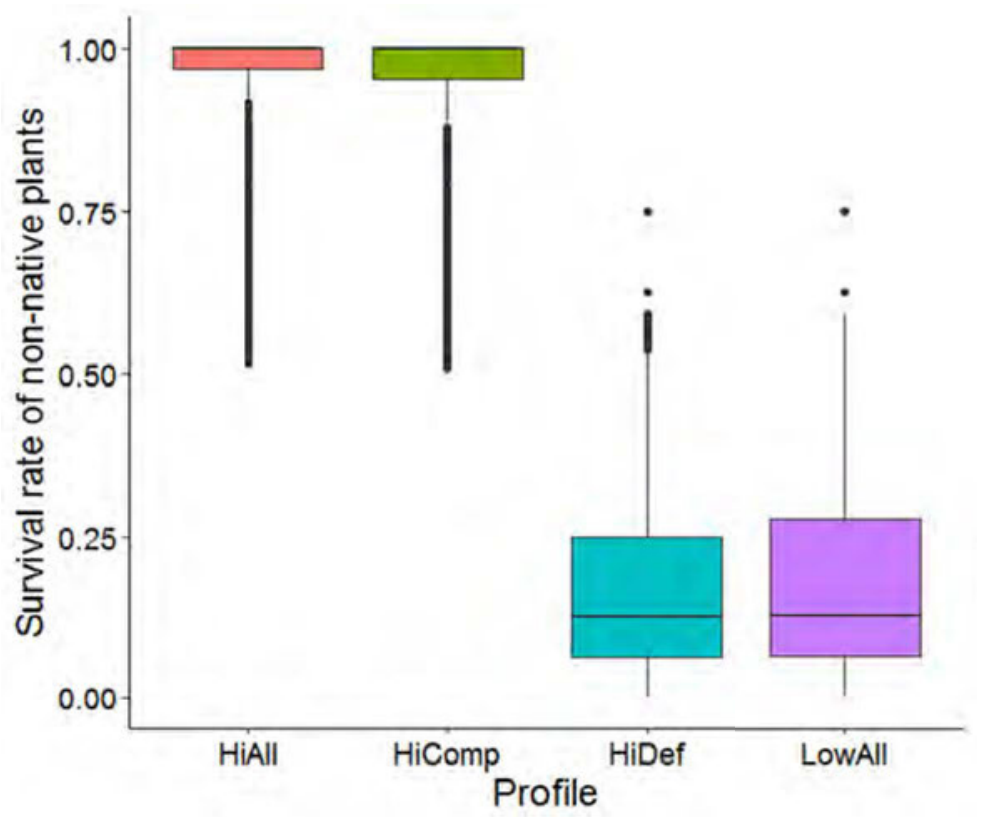

Figure 3.5. - Survival rates of non-native plants with different trait profiles. The trait profiles are: high competitive and high defensive (HiAll), high competitive and low defensive (HiComp), low competitive and high defensive (HiDef), and low competitive and low defensive (LowAll). Values were averaged over all scenarios.

Overall, weak competitors had a much lower survival rate than strong competitors. Although the populations of weak non-native competitors had an increased chance of persistence with an increasing initial population size they were not able to catch up with survival rates of strong competitors (interaction Comp:initNN in table 3.4 and fig. 3.6). This was the case even though the survival of strong competitors was significantly negatively influenced by the population size of non-natives (interaction Comp:initNN in table 3.4 and fig. 3.6).

Intensity of competition $\Theta$ had a marked negative effect on survival rates of non-native plants, especially on weak competitors (main effect Theta and interaction Comp:Theta in table 3.4 and fig. 3.7). For weak competitors, average survival dropped by $80 \%$ when comparing equal share $(\Theta=0)$ and perfect size-symmetry $(\Theta=1)$. The decrease in survival 


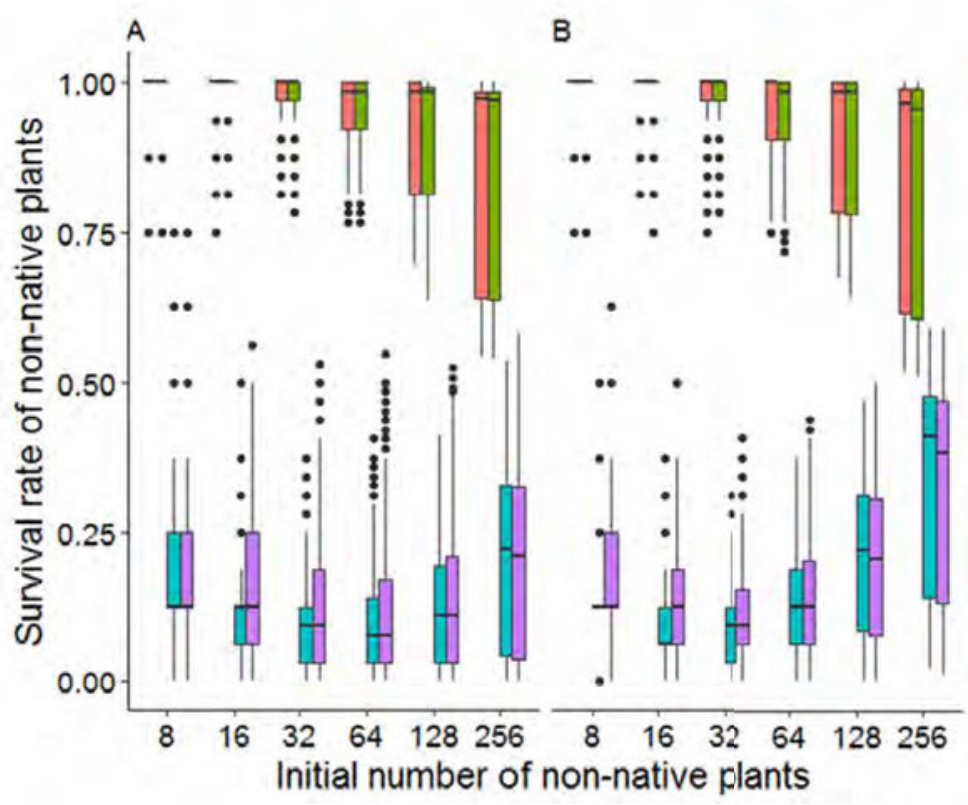

Figure 3.6. - Survival rates of non-native plants under different invasion levels in high density $(A)$ and low density $(B)$ native communities. Invasion level corresponds to initial population sizes of $8,16,32,64,128$ and 256 individuals of the non-native species. Non-natives are split into the following trait profiles (in each block from left to right): high competitive and high defensive (HiAll, orange), high competitive and low defensive (HiComp, green), low competitive and high defensive (HiDef, blue), and low competitive and low defensive (LowAll, purple).

rate was more severe in the even community mixture, as well as under high community density (interactions Comp:Mix and Comp:DensComm in table 3.4 and fig. 3.7). Strong competitors were neither visibly affected by community mixture nor by increased population densities (fig. 3.7).

In our model runs, we found that herbivores were only able to kill a plant in very early stages when they were very small. Herbivore damage increased survival of weaker competitors by a factor of up to 1.5 (main effect DensHerb in table 3.4 and fig. 3.8). Weak competitors were also usually smaller than strong competitors. For strong competitors, this effect was the other way round, i.e., presence of herbivores was on average lowering the mean survival rate (interaction Comp:DensHerb in table 3.4 and fig. 3.8). However, for strong competitors the decrease in survival rate was almost intractable, lowering survival by only $4 \%$ under complete size symmetry. Strongly defended species with $70 \%$ probability of repulsion only had a minor advantage in survival over weakly defended species with $40 \%$ probability of repulsion (main effect Def in table 3.4). 

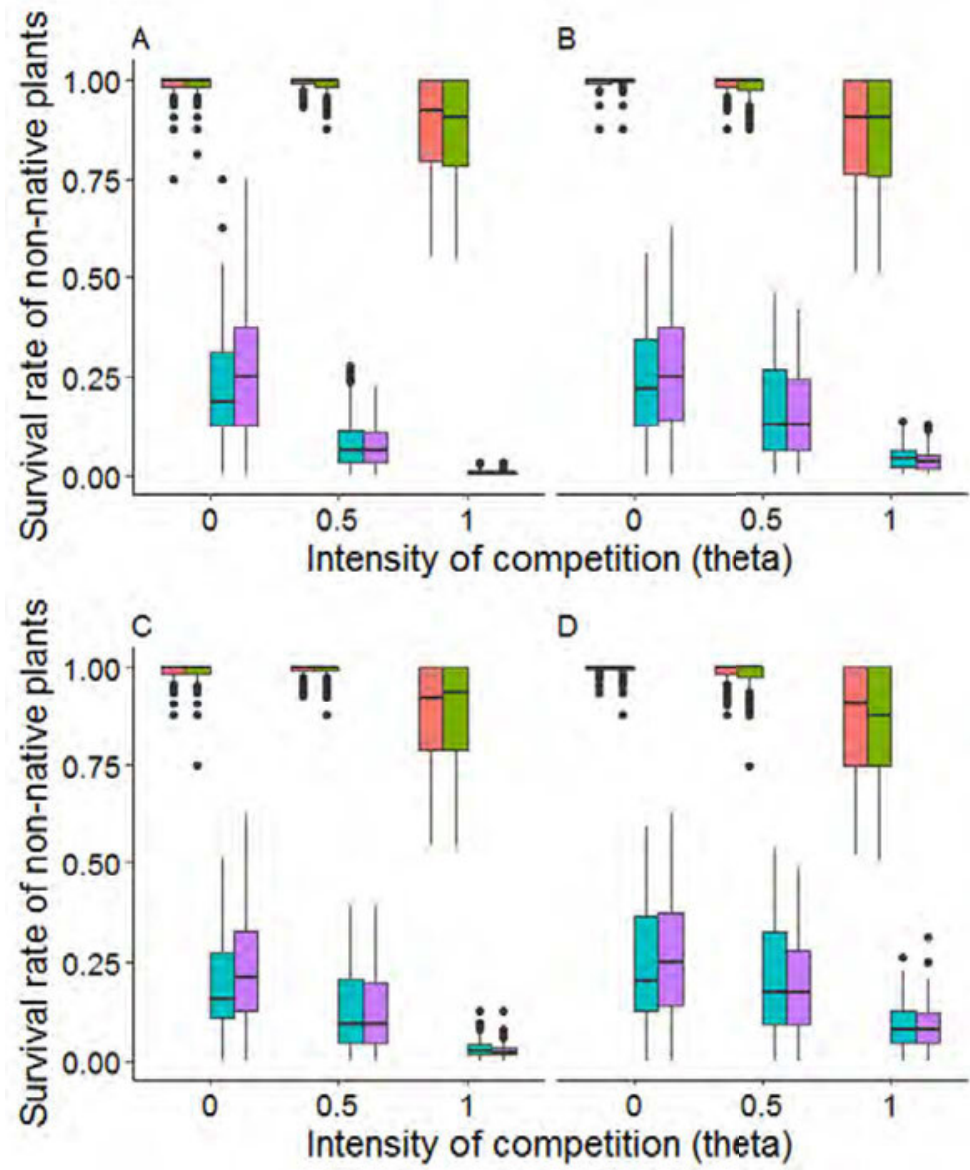

Figure 3.7. - Effect of the intensity of competition $\Theta$ on survival rates of non-native plants in different configurations of the resident community. $(A, C)$ show high density of natives, $(B, D)$ show low density of natives. Note that different initial numbers of non-natives are not separated in this figure. $(A, B)$ show an even mixture of natives in the initial community, $(C, D)$ show a mixture based on literature values of seed bank sizes. With $\Theta=0$, resources are shared among competitors regardless of their biomass, with $\Theta=1$, resources are shared proportionally to the biomass of the individual competitors. $\Theta=0.5$ reflects an intermediate stage. Non-natives are split into the following trait profiles (in each block from left to right): high competitive and high defensive (HiAll, orange), high competitive and low defensive (HiComp, green), low competitive and high defensive (HiDef, blue), and low competitive and low defensive (LowAll, purple).

The sensitivity analysis showed that the model output survival of species 2 was most sensitive to the interaction between competition index $\Theta$ and seed weight, then to maximum biomass, the interaction between the seed weights of the two competing species, the interaction between seed weight and germination probability of the same species, and the interaction of seed weight of species 1 with maximum biomass of species 2 (fig. 3.9). Germination probability and seed weight were the next most important inputs for determining survival of species 2 , together with the interaction between seed weight and repulsion of herbivores, competition index 2 , and repulsion of herbivores. Overall, species traits and 


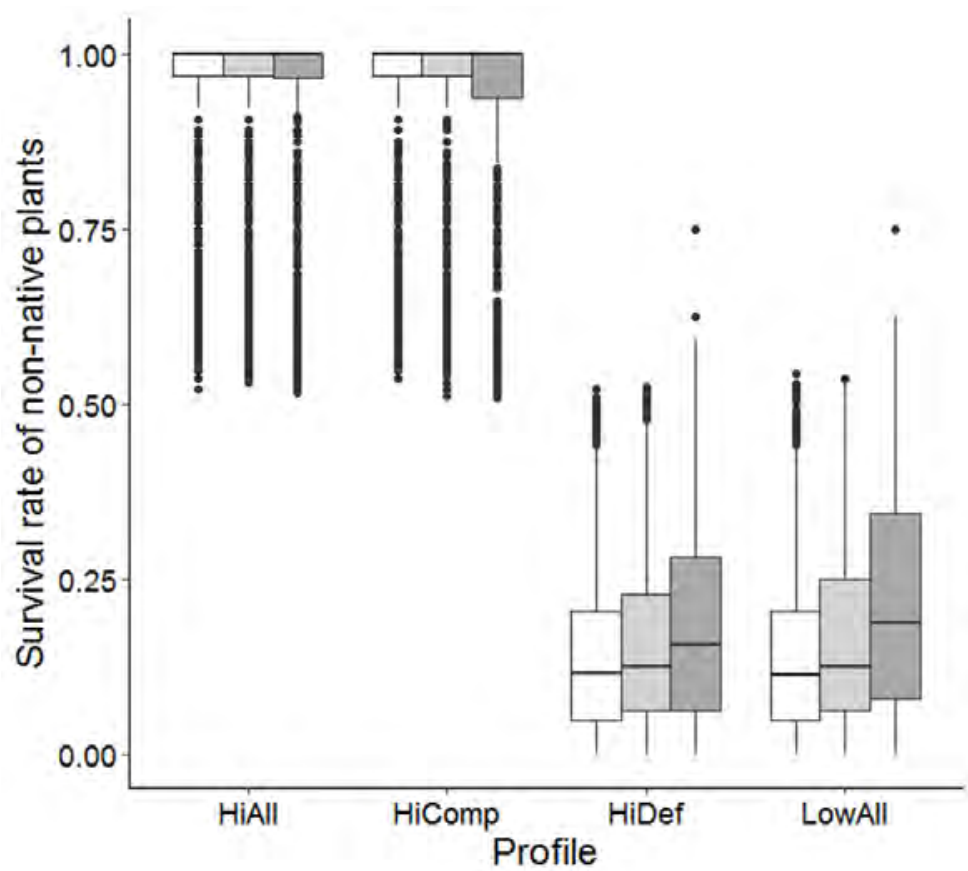

Figure 3.8. - Survival rate of non-native species at different herbivore densities. Herbivore densities: no herbivores as control scenario (white bars), 3.2 herbivores per $m^{2}$ (light grey bars), and 18.75 herbivores per $m^{2}$ (dark grey bars). Non-natives are split into the following trait profiles: high competitive and high defensive (HiAll), high competitive and low defensive (HiComp), low competitive and high defensive (HiDef), and low competitive and low defensive (LowAll).

their interactions dominated the sensitivity ranking, whereas scenario parameters such as herbivore density, initial population size of non-natives and native species density were less important. Only the competition index $\Theta$ had a large, mostly indirect influence on survival of species 2 via interactions with species traits. In contrast to biomass- and competitionrelated parameters, herbivory-related parameters such as herbivore density and repulsion of herbivores played relatively minor roles for survival of species 2 .

\subsection{Discussion}

With our model, we investigated the establishment success of different types of generic nonnative plant species in a resident native community. We targeted three aspects that might influence establishment success: trait profile of the non-native species, biotic pressures of the resident community, and the interactions between traits and pressures.

The sensitivity analysis showed that model outputs were much more sensitive to species 
Modelling plant establishment in novel communities

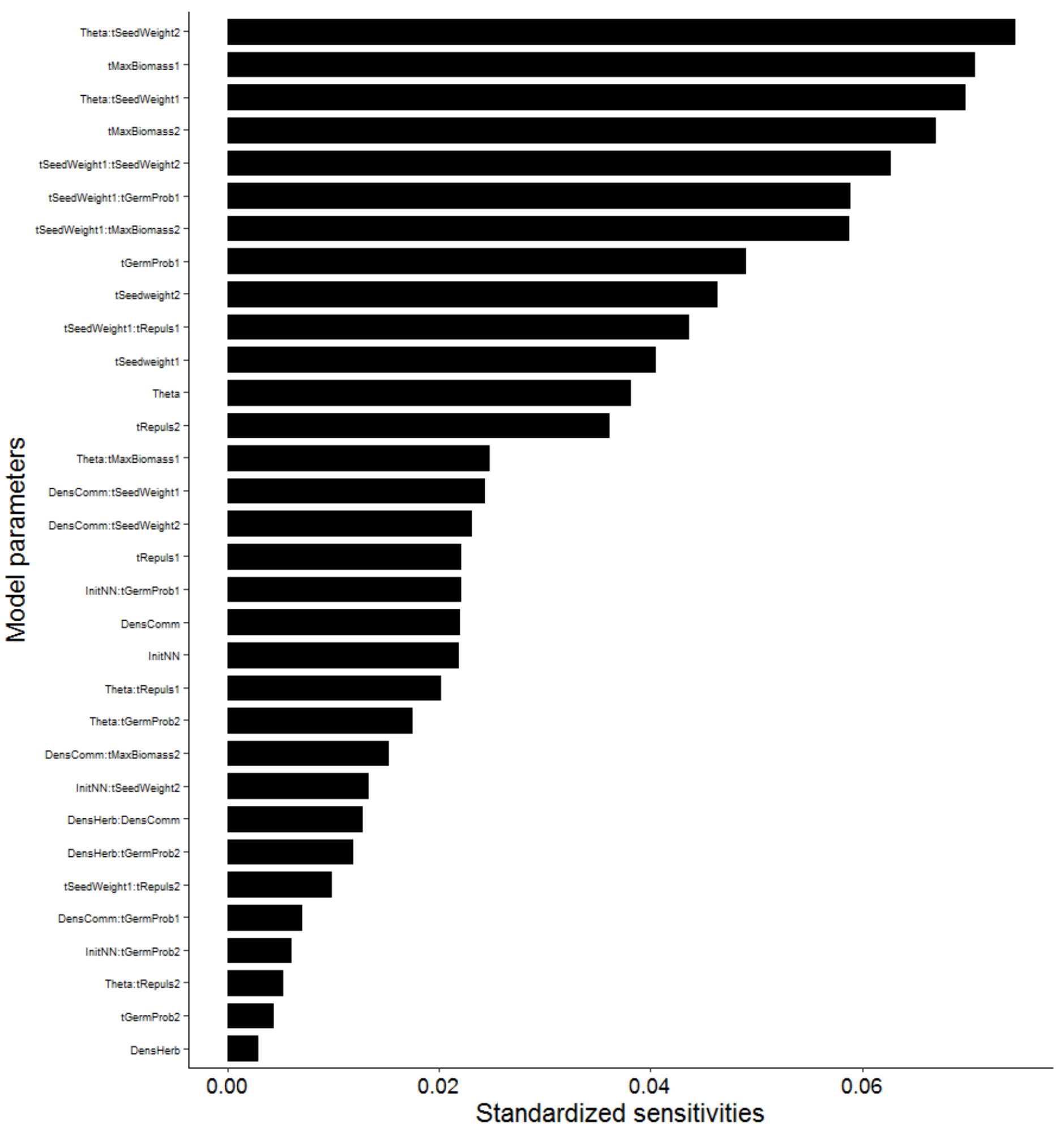

Figure 3.9. - Standardized sensitivities of model output to model parameters. Standardized sensitivities were a result of a generalized linear model of simulation results after statistical model simplification. Simulations were conducted with two species each, which corresponded to "native" and "non-native" species in the scenario analysis, but whose trait profiles were assembled here independent of real species (trait names ending with "1" or "2"). Model output was survival of species 2. Model parameters covered biotic pressures, traits (names starting with " $t$ "), and their interactions. Biotic pressures included competition index (Theta), community density (DensComm), initial number of "non-natives" (InitNN), and density of herbivores (DensHerb). Traits included seed weight (tSeedWeight), maximum biomass (tMaxBiomass), germination 68 probability ( $t$ GermProb), and repulsion of herbivores (tRepuls). 
traits and interactions between species traits and biotic pressures than to biotic pressures alone. Thus, care should be taken in the choice of experimental and model species and the traits they represent.

Competitive pressures and competitive traits exerted a much stronger influence on establishment success than pressures and traits related to herbivory. This was supported by scenario analysis and sensitivity analysis. Strong competitive traits were negatively correlated with initial density of non-natives in their effect on survival rates while a low initial number of non-natives with strong competitive ability resulted in high survival. We expect this might be due to a potential release effect from competition. These scenarios may for instance reflect distant satellite populations or the very edge of the expansion front, because there is evidence that population density on the range borders can be lower than in the core range (Brown, 1984). High survival in these conditions may translate to an effective range shift, and this is more likely if long distance dispersal is included. However, a higher initial number led to a decrease of survival rate for strong competitors. An explanation may be that the high density of individuals with strong competitive traits led to a milieu of competitive stress, provoking intra-specific self-thinning effects (Morris, 2003). In our model setting, there was no evidence of complete extinction of non-native species due to competitive stress. Thus, the high intra-specific competition decreased individual survival, but did not decrease establishment success of the populations. It would thus probably not strongly impede range expansion of non-native species. Rather, this may contribute to stabilizing the range expansion. Populations of weak competitive plants showed much lower survival at lower densities than strong competitors, implying a much lower chance of long-term establishment. Survival of weak competitors required very high initial numbers to exceed survival at low initial numbers of individuals in the population. This might be due to the sheer mass of non-native individuals that competed with the native community. In the most extreme setting 256 non-native individuals faced 300 native competitors. Thus, even if native species were the stronger competitors, a high number of weak non-native competitors might be able to overcome the biotic resistance, a trend that has already been observed in studies of intercontinental invasions (Lockwood et al., 2005; Simberloff, 2009). This effect was clearly observable although there was only a small difference between seed weight of weak non-native species and native competitors. We expect the effect to be even more pronounced if the difference in seed weight was larger. 
The advantage of strong competitors compared to weak competitors persisted in community settings with more intense community-borne competitive stress, i.e., at higher community density and species mixtures with a higher proportion of strong competitors. Higher community density results obviously in a higher number of competitors for each individual plant and thus in most cases a lower amount of resources that can be captured by any plant. For the community mixture, an even number of individuals of all species led to a community with a much larger proportion of strong competitors, i.e., P. lanceolata, the largest native species in our setting, than in the seed bank-based mixture. However, since strong nonnative competitors were characterized by twice the seed weight of $P$. lanceolata they were probably not massively impacted by numbers of competitors or community mixture. We observed this effect in the preceding empirical experiment as well, where strong non-native competitors were massively dominating the native community (Radny et al., 2018).

These results indicate that a range shift should be more effective for such plants that are strong competitors relative to species of the receiving community, provided that their seeds can reach a novel habitat. For intercontinental invasions competitive strength is one of the major explanations of invasive success (Vilà and Weiner, 2004). In the context of climate-change induced range expansion, this might become just as important or even more important, because changes in the microclimatic regime of habitats beyond current range borders may weaken the currently strong resident competitors and thus increase invasibility of communities (Alexander et al., 2016; Bauer, 2012; Stanton-Geddes et al., 2012). However, the very low survival rates of weak competitors in our model may overestimate the negative impact of community competition on weak competitors in reality. For instance, in our preceding experiment (Radny et al., 2018), drop-out rate of weak competitors was almost zero while in the model weak competitors responded very drastically to increased competitive pressure from the community in form of increased density, mixture and intensity of competitive asymmetry $(\Theta)$. Probably, in our model, we underestimated the abilities of weak competitors to avoid or tolerate competitive pressure from other species. This might be partly due to the implementation of competition with the Zone-of-influence approach. For theoretical models of competition between plant individuals, the Zone-of- Influence approach has been used many times at different degrees of complexity (e.g. Berger et al., 2008; Lin et al., 2012; Weiner and Damgaard, 2006). Despite several simplifications, it is a straightforward and comparatively easy method to investigate competition (Berger et al., 
2008). However, most of these models address monocultures of species and thus implement the same type of interaction, i.e., degree of asymmetry $\Theta$, for all individuals. Interspecific interactions may be different though from intraspecific interactions due to many different mechanisms. This may not only imply differences in interspecific and intraspecific $\Theta$, but also different $\Theta$-values depending on the identity of the focal species (Connolly and Wayne, 1996). Such mechanisms include for example allelopathy (Bais et al., 2003) or adaption to the competitive disadvantage, e.g., development of shade tolerance in trees (Dislich et al., 2010). Additionally, following the parsimony principle we have not yet considered facilitative interactions in this model, although there are potentially strong impacts of facilitative interactions in plant communities (e.g. Lin et al., 2012). Of course, parameterizing different competition types for all possible interaction partners in our five-species system would require a lot of data, which were not available in our case, and has also been attempted in only very few comparable cases thus far. Thus, we strongly advocate for the extension of multispecies models to incorporate different forms of neighbourhood interactions not only as negative (competition) or positive (facilitation) interactions, but also accounting for different intensities of inter- and intraspecific interactions. This approach will require enhanced efforts in the collection of adequate data for parametrization, but we expect a much better understanding of multispecies systems from such approaches (Svenning et al., 2014).

The effect of herbivory was comparatively small in our model. Accordingly, defensive strength did not play an important role for survival. This may be due to the indirect influence of biomass on survival via its effect on plant growth (fig. 3.10) combined with the fact that model herbivores only consumed absolute amounts of biomass. This means that large plants suffered relatively less from herbivory than small plants. In extra simulations, where herbivores consumed relative amounts of plant biomass, defence traits were much more important for survival than in the standard simulations (see appendix A). Herbivory has been reported to influence range expansion and invasions, i.e., in spatial pattern and speed (Fagan et al., 2005; Herrero et al., 2016), yet it is unlikely that herbivory may entirely block establishment of novel species (Jeschke et al., 2012; Levine et al., 2004). However, although herbivory as a single factor may not pose a hard barrier to establishment, studies found herbivory to be an important interacting effect under competition through altering the competitive impact of individuals (Huang et al., 2012; Kim et al., 2013; Kuijper et al., 


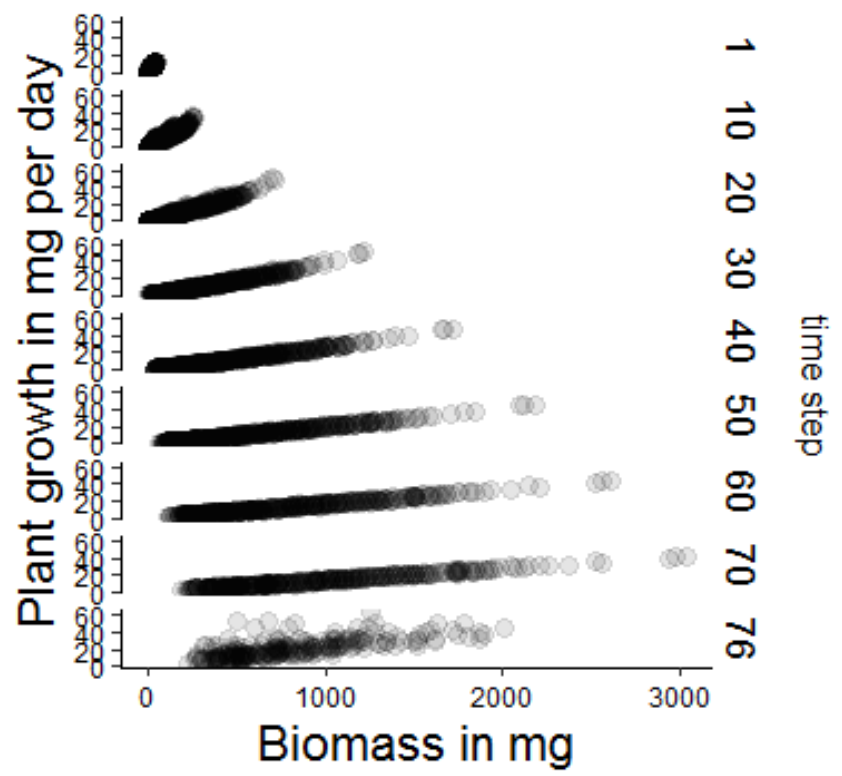

Figure 3.10. - The relationship between plant growth and plant biomass in the model. Each point cloud represents one daily time step in the model as indicated on the righthand side. Plant biomass has a strong influence on plant growth in the model.

2004). Our model results support such an interaction between herbivory and competition, where weak competitors showed increased survival under herbivory, especially with higher densities of herbivores. We suspect that weak competitors benefited from being small relative to their neighbours - either due to initial small size or due to cumulated negative effects on biomass gain from competition. As sharing of the contested resource can depend on the relative biomass of the competitors, loss of biomass due to herbivory can reduce the resource capture of strong competitors, so that more resources are left to neighbouring weak competitors than in scenarios without herbivory. In our model, the minimum amount of resource uptake for maintenance of metabolism and thus survival is directly related to current biomass of the individual (Brown et al., 2004). Thus, even a relatively small increase in resource capture can increase survival of smaller plants. Of course, our model did not capture the full complexity of possible herbivore impacts on plant distribution and range expansion. Body size of herbivores and timing of herbivory have been shown to differentially affect plant biodiversity (Kim et al., 2013; Olff and Ritchie, 1998), as well as presence or absence of specialist herbivores (Joshi and Vrieling, 2005; Lakeman-Fraser and Ewers, 2013). Our extra simulations on relative herbivory (see appendix A) indicate that it may be worthwhile to explore a greater range and resolution of herbivory implementations once the respective data become available for parameterization. Future model extensions 
could reflect these factors as well as plant internal mechanisms such as compensatory growth (Lu and Ding, 2012) and increase of defence mechanisms (Strauss and Agrawal, 1999). However, even with our simple model design, we found a significant effect of herbivory - although not the expected global decrease of survival, but an indirect effect through harming the competitors. This indicates that herbivory effects may sometimes be overseen when they are not turning out as expected and that this might also be a reason for contradicting results of similar studies (Jeschke et al., 2012; Levine et al., 2004). For further developments of local competition models in a community context, we advocate to develop approaches that include tolerance strategies. In the frequently used ZOI approach this could be realized by an asymmetry index that is sensitive to the identity of interaction partners.

\subsection{Conclusions}

We conclude that traits related to competitive strength of species can change the effectiveness of biotic resistance from resident competitors and should be taken into account when attempting to predict establishment success of range expanding species.

Where the impact of herbivores is of minor importance, strong defence traits do not result in an apparent advantage as compared to weak defence. However, herbivory might have a stabilizing effect on competition and thus should not be neglected when analysing range expansion dynamics.

This model may serve as a basis for future large-scale models, where dispersal should be considered as a third important trait to describe range expansion. 



\title{
4. Right here, right now? - Macrosystems ecology as conceptual framework for modelling climate change-induced range dynamics
}

This chapter has not been part of a peer-reviewed publication to date, but is left open for publication in the future. JR established the concept and wrote the text. KM improved the manuscript with valuable comments.

Referenced literature is included in the full thesis literature.

\begin{abstract}
The response of plant range dynamics to climate warming is linked to processes on different spatial and temporal scales as well as complex interactions across scales. The prediction of species range dynamics relies on three fundamental questions addressing future local habitat suitability, connectedness between localities, and establishment success in novel habitats. We propose a model concept for the prediction of species range dynamics and embed the model concept in the macrosystems ecology framework. Based on this framework, we identified the relevant processes for predicting the response of species range dynamics to climate warming on a species level (macroscale), a metapopulation level (mesoscale) and a population level (microscale). In line with macrosystems ecology, we also considered interactions between elements (holons) on the same level, cross-scale emergences and cross-scale interactions. On the species level at the macroscale, the overall range dynamics pattern depends on the ratio of extinction and colonialization. The range dynamics pattern at the species level emerges from population growth and dispersal on the mesoscale. Pop-
\end{abstract}


ulation growth emerges from individual performance as a result of local biotic interactions on the microscale. Cross-scale interactions, especially the impact of climate warming on local habitat suitability and species interactions, and feedback loops, such as the impact of vegetation cover on regional climate conditions, have to be considered for a reasonably realistic prediction of future ranges. While the overall pattern of interest emerges on a macroscale, it is crucially dependent on biotic interactions, operating on a microscale. In complex communities, multispecies interactions on the same and higher trophic levels can be of crucial importance for local dynamics and should not be neglected. The implementation of such a model will be very demanding in terms of parametrization and technical approach. Using hybrid models that combine systems models and individual-based models on different scales and the increasing availability of data and big data handling techniques will potentially allow for the implementation of such a model in the near future.

\subsection{Introduction}

Understanding range shift dynamics of plants under climate change can help to assess vulnerability of species and ecosystems and give advice for conservation efforts (Ehrlén and Morris, 2015; Freeman et al., 2018). Predictions of range dynamics are particularly challenging, because they require addressing broad spans of spatial, temporal and organizational scales. Here, we propose a model concept that meets this challenge by integrating the recently proposed concept of macrosystems ecology and individual-based modelling.

To predict the range dynamics of a given species, it is necessary to know: (a) which regions will become (un)suitable habitats for that species due to changes of climatic conditions, (b) whether suitable novel habitats can be reached by that given species in a sufficient time frame, and (c) whether that species can establish self-sustaining populations in these habitats. Simulation models are a valuable tool to approach these questions through extrapolation of current patterns to future developments. With varying richness of detail, several types of simulation models have been applied during the last decades to predict species distributions and range dynamics, including correlative models and process-based approaches (Dormann et al., 2012).

Correlative models are a standard approach for modelling species distributions (Araújo and 
Pearson, 2005; Elith et al., 2010; Kearney et al., 2010; Tyberghein et al., 2012). For example, bioclimatic envelope models are correlative models that examine the climatic conditions within a focal species current range to assess its climatic requirements. When applying different scenarios of climate warming, these models can identify those regions where a given species might meet similar climatic conditions in the future as in its current range. The basic assumption of these models is the dominant role of climatic conditions on species distributions (Pearson and Dawson, 2003).

Recently, these models have been challenged because important processes are not or only implicitly considered (Araújo and Peterson, 2012; Pearson and Dawson, 2003). Referring to the guiding questions stated in the beginning, strictly correlative models often have shortcomings in addressing the following features: (a) - habitat suitability: adaptation of individuals and evolution of the species to changing conditions, (b) - translocation: dispersal capacity and natural obstacles or anthropogenic landscape fragmentation, and (c) - population establishment: only an implicit consideration of biotic interactions (Heikkinen et al., 2006; Pearson and Dawson, 2003). These factors can crucially impact population and range shift dynamics on a species level and may lead to false estimations of climateinduced range shift dynamics (Brooker et al., 2007; Guisan and Thuiller, 2005; Harvey et al., 2010; Van der Putten, 2012). Adaptation of a local population to changing conditions can attenuate habitat loss caused by climate warming, provided adaptation can keep pace with rates of climate warming (Jump and Penuelas, 2005). Natural obstacles such as mountain ranges or anthropogenic landscape fragmentation can impede dispersal of propagules to suitable novel habitats (Mestre et al., 2017; Saura et al., 2014). Biotic resistance may pose a sincere barrier to establishment of species or slow down the velocity of range shift (Levine et al., 2004). A lack of mutualists or enemies can exert a massive effect on establishment success of species in a novel range (Van der Putten, 2012).

One reason why bioclimatic envelope models often lack or only implicitly consider the features described above is the inherent scale mismatch. Such mismatch arises from different extents and detail (grain) of the observations (Schneider, 2001). For example, despite what some voices utter in popular media, observing the weather of one season in a given region does not allow for conclusions about global climate warming rate (see also section 4.3.3 climate expression across scales). Climate and climate warming are observed on a global or continental scale and over several, most commonly three decades (IPCC, 2014). 
Bioclimatic envelope models operate with similar, e.g. continental extent (e.g. Bakkenes et al., 2002). Yet, at such coarse scales these models cannot capture valuable information about microclimatic conditions, although such microhabitats can form important refuges and stepping stones for persistence and dispersal of species (Franklin et al., 2013; Hannah et al., 2014).

. Dispersal and biotic interactions operate on much smaller scales than climate. Compared to the extents of climate observations, average dispersal distances and hence gene flow between local populations often only comprise a tiny fraction of the observed system, even under the assumption of naturally occurring long-distance dispersal events (Nathan, 2006; Soons and Ozinga, 2005). In fact, estimations of velocity of range border shift towards the poles was averaged to $6.1 \mathrm{~km}$ per decade (Parmesan, 2006), or $16.9 \mathrm{~km}$ per decade (Chen et al., 2011).

Moreover, biotic interactions such as competition or enemy attack also operate in the very immediate surrounding of an individual plant and often in a matter of minutes, e.g. grazing by herbivores. Where correlative models consider such fine-grained processes only implicitly, they are prone to miss valuable information. Therefore, for applications of purely correlative models, users have to be aware of these pitfalls in order to avoid or at least identify potential sources of bias associated with this approach (Araújo and Peterson, 2012; Heikkinen et al., 2006).

Instead of the top-down approach as used in correlative models the alternative bottom-up approach explicitly models local processes to asses large-scale patterns, for example through individual-based modelling (Grimm and Railsback, 2005; Grimm et al., 2005). Such smallscale process-based models capture processes on a very fine grain. For example, individualbased models may capture immediate herbivore attack to a single plant within one day. However, the computational efforts are unreasonably high when trying to implement such fine-grained processes with brute force to the broad scales required for predicting species range shifts under climate change. Extrapolation from a small-scale source model to a far larger-scaled target model offers an alternative to brute-force modelling of a large number of small-scale processes. This interpolation of information from smaller to larger scales is commonly known as scaling-up (Lischke et al., 2007). With respect to modelling, the term scale usually describes the grain and extent of a model while the term level refers to ecological units. Comparably to scales, levels allow for varying richness of detail in 
observation in a hierarchically ordered way, e.g. population, community, ecosystem and biosphere.

For an extrapolation-based scaling-up, the different scales are in most cases hierarchically ordered during model construction, with grain and extent based on ecological and databased considerations. Lower levels correspond with fine grain and small extent and higher levels correspond with increasingly coarse grain and large extent (Lischke et al., 2007). With classical hierarchical approaches, lower levels, i.e. processes at scales with a smaller extent and finer grain, inform higher levels, while higher levels, i.e. at scales with broader extent and coarser grain, provide constraints on the lower levels (Heffernan et al., 2014; Wu, 1999). Additional to this classical view, some processes on lower levels have the potential to directly alter processes on the higher level. For example, massive outbreaks of bark beetles can substantially change composition of tree species (emergence from higher to lower level) and thereby influence climatic conditions on the higher level through alteration of carbon sequestration (Raffa et al., 2008). Such impacts from lower to higher level result often in non-linear behavior of the system (Peters et al., 2004; Wu and David, 2002). Crossscale interactions from lower to higher scale and vice versa are detected in more and more systems (Guisan and Thuiller, 2005; Levin, 1992; Peters et al., 2007; Soranno et al., 2014). Where such feedbacks are not accounted for, there is a threat of severe prediction errors (Heffernan et al., 2014). This reflects the claim that the concept of scale is not inherent to the system of interest and thus the different scales are not necessarily neatly separated and hierarchically nested (Levin, 1992; Lischke et al., 2007). Scaling rather is an assignment by an external observer - the modeler or field scientist - and based on the best-possible representation of relevant processes or data availability (Wu and David, 2002).

Macrosystems ecology is a new concept that explicitly integrates cross-scale feedbacks, emergence and interactions (Heffernan et al., 2014; Levy et al., 2014). Consideration of cross-scale interactions opens the possibility to account for linear and non-linear responses on the level of interest (Peters et al., 2007). Thus, macrosystems ecology might be a promising concept to alleviate scaling problems in modelling.

Here we propose an individual-based model structure for estimating range shift dynamics of plant species based on the concept of macrosystems ecology. Firstly, we give a brief introduction to the concept of macrosystems ecology. Secondly, we apply this concept 
to propose a conceptual model framework for the characterization of patterns of climate change-induced range shift of plant species. To this end, we formulate a guiding question and follow this question through the organizational levels of the model and identify some of the potential feedback loops that may emerge from cross-scale interactions. Finally, we discuss some associated challenges and sources of bias for implementation of such a model.

\section{Glossary}

Climate Long-term average weather conditions in a specific area (WMO, 1966).

Climate change Differences from the long-term average weather conditions (WMO, 1966). Current observed increase of global mean surface temperature is referred to as climate warming.

Colonialization Establishment of a self-sustaining population in a location formerly not inhabited by the focal species.

Cross-scale emergence Patterns and processes occurring on a broader scale as result from processes operating on a smaller scale (Heffernan et al., 2014).

Cross-scale interactions Factors operating on one level with impacts on factors operating on another level (Heffernan et al., 2014).

Level of observation Describes the level at which individuals of a species are organized in a model, here: community, metapopulation, and species. Based on hierarchy theory, the levels are ordered as following: lower level - local population in a community, medium level - metapopulation in a region, and higher level - species over the entire species range.

Extinction Local disappearance of a population from a habitat up to global disappearance of the species.

Interaction web Complete set of interactions in a local community, i.e. mutualistic and antagonistic interactions within and across trophic levels.

Macrosystems ecology The study of ecological phenomena at broad scales, e.g. regions to continents, and their interactions with phenomena at smaller scales, e.g. plots or organisms (Fei et al., 2016; Heffernan et al., 2014).

Microclimate Climatic conditions close to the surface on a local scale.

Range of a species All geographical area(s), where persistent, self-sustaining populations of this species occur. 
Scale Spatial and temporal extent relative to grain of the variable under observation, where extent describes the range of measurement and grain the resolution of observation (Schneider, 2001).

Weather State of the atmosphere at a particular time, as defined by the various meteorological elements (WMO, 1966).

\subsection{Hierarchy theory and macrosystems ecology}

During the last decades, ecological problems and interrelationships have increasingly been acknowledged to be large-scale questions (Peters et al., 2008). Especially when regarding global processes such as loss and homogenization of biodiversity or global climate warming, there is a pressing need to understand systems on a global scale or "macroscale" in order respond to associated challenges and opportunities. However, large-scale patterns and processes can hardly be fully understood when not seen in the context of local processes (Peters et al., 2008, 2004; Soranno et al., 2014).

Technical development facilitates the large-scale data collection that is required to investigate large-scale patterns (Sandel and Smith, 2009) and processes through ever improved remote sensing methods (Wiegand et al., 2000), as well as progress in big data computing (Farley et al., 2018; Soranno and Schimel, 2014). For example, it is possible to use earth observation systems such as radar for tracking annual migration behavior of species rather than only tracking individuals (Kelly and Horton, 2016). Consolidation of many small datasets in form of an open data repository can further improve data availability (Cheruvelil and Soranno, 2018; Kress, 2019). Although usage and combination of many small or extensive datasets is far from being trivial (Soranno and Schimel, 2014), these improved methods in data sampling and analysis can open the door to understand vast and complex systems (Chave, 2013). Additionally, consolidation of many small-scale datasets as well as citizen science into open-access data repositories offers a valuable source of knowledge for many scientists (Levy et al., 2014).

In light of pressing broad-scale questions and increasing availability of data, new frameworks for understanding ecological networks arise. Macrosystems ecology, proposed by (Heffernan et al., 2014), is such a framework derived from hierarchy theory. Hierarchy theory is based 
on the idea that complex systems to a certain degree can be decomposed into smaller systems (scaling-down) or arise from those smaller systems (scaling-up) (Wu, 1999). This results in a vertical structure (levels), and a horizontal structure (holons) within each scale. Holons are group components with high interaction frequency or strength, but weaker or less frequent interactions with other holons or components of holons are possible (Lischke et al., 2007; Wu, 1999).

Strong interactions imply strong coupling between the subsystems, while weaker interactions imply loose coupling. Since interaction between components is an inherent feature of systems in general, fully detached components are not expected. This is why ecological hierarchies are described as "nearly" but not "fully decomposable" (Wu, 1999). Level building is based on process rates of the contained systems, for example cycle time or response rate. The expectation is that higher levels are characterized by slower process rate and lower levels are characterized by faster process rates. This results in the perception that on an intermediate level very slow processes of a higher level appear to be stationary, e.g. climate change or plate tectonics, while very fast processes of a lower level, e.g. individual metabolism as response to daily weather conditions, are observed as "noise".

Often three-level systems are considered, e.g. a macro-, meso-, and microscale, or a focal level $n$, a higher level $n+1$ which could set context and constraints, and a lower level $n-1$, providing information on underlying mechanisms (Wu, 1999). Depending on the question or system at hand, this can also be extended to model frameworks with a greater number of levels. For example, Mackey and Lindenmayer (2001) use a four-level system - species, population, community and individual - to adequately capture relevant environmental and behavioral factors for description of distribution of the Leadbeater's possum (Gymnobelideus leadbeateri ). Especially in multispecies systems, a range of layers are introduced to deal with spatial and temporal mismatch (Heffernan et al., 2014).

Macrosystems ecology builds on this framework, accounting for interaction connections of components within the same scale and across scales (Heffernan et al., 2014; Peters et al., 2008). Cross-scale connections include emergence and interactions. Emergence occurs where accumulated processes from smaller scales form patterns and processes on broader scales. Interactions occur where components of one scale influence components and processes operating on another scale. Considering cross-scale interactions allows for capturing 
behavior, which would not be obvious when applying single-scale models or models with multiple, but independent scales (Peters et al., 2007).

Cross-scale connections in the form of emergence or interactions are important for being able to capture potential non-linear responses from one scale to another, such as sudden, unexpected events (Peters et al., 2004). Especially in models on global climate change, such sudden changes, referred to as tipping points, are to be expected. Tipping points are accumulating local or regional changes, which provoke changes in the global climate system when reaching a certain threshold (Lenton et al., 2008). A prominent example is the decreasing sea ice cover in the Arctic regions. The decrease of that ice cover goes along with decreased albedo and thus increased heating of the ocean, potentially giving momentum to other adjunct tipping points (Shimada et al., 2006). Another potentially nonlinear interaction can occur between species occurrence and regional climate. Vegetation cover was shown to have measurable impacts on regional climate (Copeland et al., 1996; Foley et al., 2000; Green et al., 2017). In fact, tropical rain forests and boreal forests even affect the global climate system (Bonan and Shugart, 1989; Lenton et al., 2008; Malhi et al., 2009), e.g. as carbon sink and large-scale evapotranspiration control (Chapin et al., 2008). Such interactions can result in reinforcing feedback loops (Chapin et al., 2008). For example, when landscapes lose parts of their vegetation cover related or partially related to climate warming, exactly this loss can further enhance unfavorable climate development ( $\mathrm{Ci}$ and Yang, 2004; Geist and Lambin, 2004; Malhi et al., 2009; Rosenfeld et al., 2001).

The arising biocomplexity that arises from accounting for cross-scale emergence and interactions poses many challenges to modellers. One challenge of course concerns theoretical considerations, e.g. which components to include or not and how to deal with unexpected interaction relationships (Levy et al., 2014; Williams and Jackson, 2007). But there are also practical challenges to be faced. That includes provisioning of data (Rüegg et al., 2014) as well as being able to translate available data between scales (Sandel and Smith, 2009), and, with increasing importance, between disciplines (Cheruvelil and Soranno, 2018; Goring et al., 2014). The complexity of macrosystems will require a high level of teaming-up of experts from ecology, climatology and hydrology, but also from more technical fields, such as mathematics and computer sciences. 


\subsection{Climate warming-induced range dynamics in an macrosystems ecology framework}

We present a model concept for simulating climate change-induced range expansion embedded in a macrosystems ecology framework and using an individual-based modelling approach. The scale-explicit structure of the macrosystems ecology framework including cross-scale emergence and cross-scale interactions reflects the bottom-up approach of individual-based models. Therefore, it is straightforward to integrate the macrosystems ecology framework with an individual-based modelling approach. For this integrated model concept, we first identify the central question of interest for the entire model, the "guiding question". We then follow the scales from macro- to microscale, addressing the ecological units from higher level of observation (species) to lower level of observation (community). For each level and scale we describe the major entities and processes that operate on that level, as well as feedback loops that may arise through the interactions between levels. We distinguish between species-intrinsic and species-extrinsic components at each level (fig. 4.1). In a third step, we highlight potential cross-scale emergences (bottom-up) and cross-scale interactions (top-down).

\subsubsection{Definition of the guiding question}

The guiding question for this model concept will be: Which patterns of range dynamics will a focal plant species show under expected future climate warming?

For the individual-based simulation model, we propose to implement three levels at corresponding spatial scales (fig. 4.1). We adopt the concept of organizational levels from a species-centered perspective, i.e. the whole species at the highest level, a metapopulation of the species at the intermediate level, and the local community that accommodates a population of that species at the lowest level. For the macroscale (species level), the species' entire range is regarded, e.g. Western Europe. The mesoscale (metapopulation level) covers a regional extent and captures population dynamics and exchange between populations. The microscale (community level) considers individuals and is operating in a single patch.

In the following, we will describe how range dynamics patterns of species emerge at the macroscale as result of processes on the mesoscale and microscale and follow the pattern 
4.3 Climate warming-induced range dynamics in an macrosystems ecology framework

formation from the higher (broader) to the lower (smaller) organizational levels (scales). Then, we will highlight some aspects of how these organizational levels are coupled by cross-scale interactions and emergences. 
Figure 4.1 - Spatial level, pattern emergence and major processes for the integrated model. Under major processes, blue boxes (range dynamics; metapopulation dynamics; local population dynamics) mark output variables of the respective organizational level, i.e. informers for the next higher level. Green boxes (evolution, adaptation potential, gene flow, dispersal, reproduction and individual fitness) mark species intrinsic processes. Orange boxes (climate, landscape configuration, microclimate, habitat suitability, enemies \& competitors, and mutualists \& facilitators) mark external factors, influencing plant growth and fitness. Arrows account for expected direction of interaction, but not interaction strength, as this is very specific for a focal species. Indirect connections are not included in the chart for clarity. For example, local landscape configuration impacts dispersal. As dispersal impacts metapopulation dynamics, there is an indirect connection between landscape configuration and metapopulation dynam$i c s$, but the underlying mechanism is the direct impact of landscape configuration on dispersal. Pictures illustrating the macroscale (Central Europe) and mesoscale (Black Forest) were created with Google Earth Pro on January, $26^{\text {th }}$ 2019.

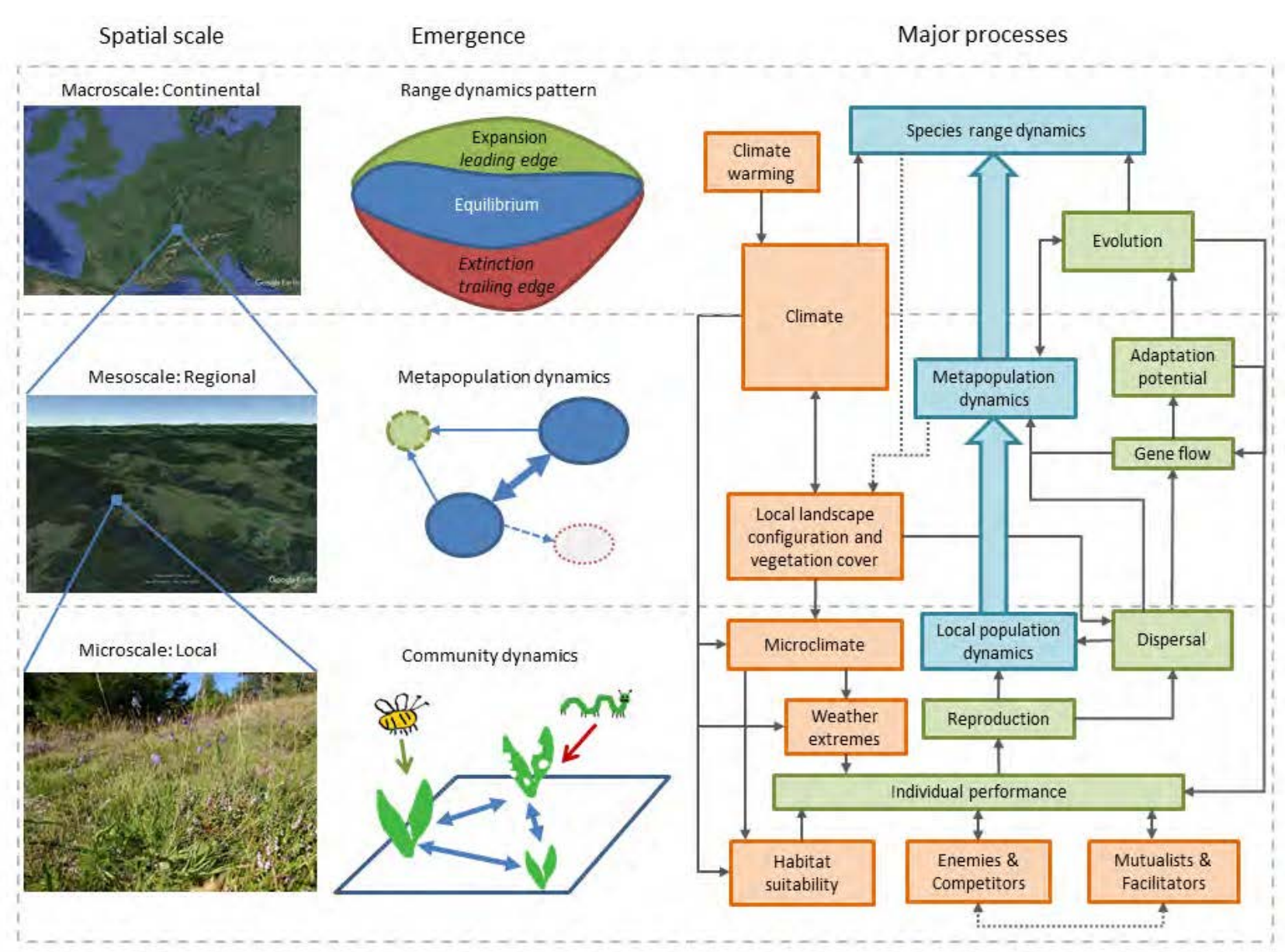




\subsubsection{Structure of the model concept}

\section{Macroscale: Species level}

The pattern of interest is observable on a macroscale and expressed as the variation of species range borders over time.

Different patterns of range dynamics emerge from variation in two types of events, i.e. colonization and extinction. Here, we define colonization as the establishment of selfsustaining populations in regions and habitats that have not been occupied by the species at initialization of the model. The second event is extinction, here defined as the disappearance of self-sustaining populations in a formerly inhabited region or habitat.

Assuming that range dynamics patterns emerge from colonialization and extinction events, there are four possible types of range dynamics, based on the ratio of colonialization and extinction events (fig. 4.2).

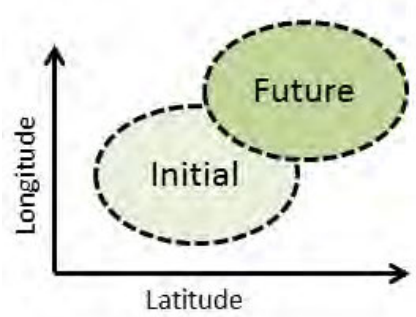

(a) Range shift

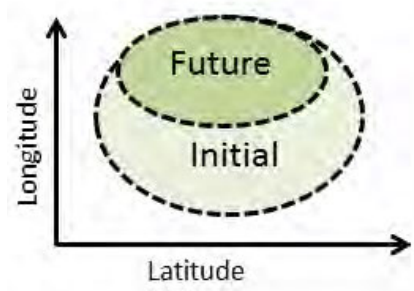

(c) Range contraction

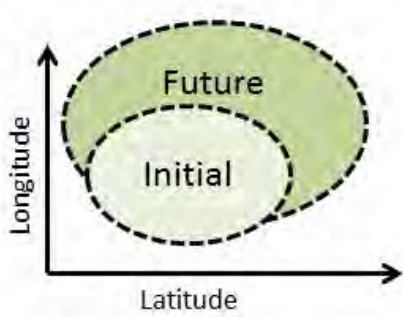

(b) Range expansion

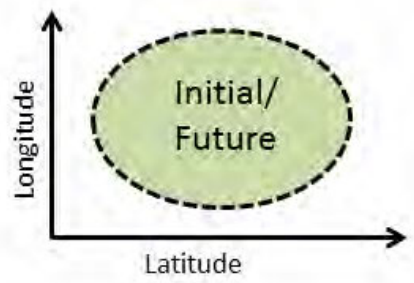

(d) No border shift

Figure 4.2. - The ratio of local colonialization, i.e. establishment of novel populations, and extinction, i.e. disappearance of existing populations, can result in different types of range dynamics: (a) range shift: colonialization and extinction events are largely in balance; (b) range expansion: colonialization events outmatch extinction events; (c) range contraction: extinction events outmatch colonialization events; (d) no extinction or colonialization events. The same scheme can be applied to altitudinal range dynamics. 
Range shift occurs, when colonialization events on one range limit are in balance with extinction events on another, often opposing range limit. Here, the overall extent of the range remains stable, but the geographic location changes (fig. 4.2a). Such movements have been observed on mountain slopes, when species colonize habitats at higher altitudes, but simultaneously disappear from habitats at lower altitudes (Colwell et al., 2008; Freeman et al., 2018; le Roux and McGeoch, 2008; Lenoir et al., 2008; Peñuelas and Boada, 2003). Range expansion emerges where colonialization events occur more often than extinction events, i.e. the extent of the entire range is increasing (fig. 4.2b). Contrary to range expansion, range contraction is the result of a higher number of extinction events as compared to colonialization events (fig. 4.2c). Here, the overall extent of the species initial range is shrinking. This pattern is often related to a failure to adapt or migrate to changing conditions in current habitats (Freeman et al., 2018; Jump and Penuelas, 2005; Zhu et al., 2012). A zone of colonialization events is commonly called the leading edge, while the zone of extinction events is called the trailing edge. Of course, some species might show neither colonialization nor extinction tendencies, persisting with relatively stable range borders (fig. 4.2d). Such an equilibrium state might be related to a species' high capacity of adaptation or tolerance against novel conditions (Berg et al., 2010; Jump and Penuelas, 2005). Hence, on this level the model operates as species distribution model, recording presence or absence of self-sustaining populations. Depending on the species under observation, the spatial extent should be chosen appropriately. For example, to capture range dynamics of beech (Fagus sylvatica), is might be reasonable to have the models' spatial extent spanning all over Europe, while for rare or highly specialized species such as the edelweiss (Leontopodium nivale) in the Alps, a fairly smaller spatial extent is sufficient. Several models exist that observe exclusively one edge of a species range. Nevertheless, it is advisable to observe as much of the entire range as feasible, in order to capture the entire pattern (see fig. 4.2 and table 4.1) as well as capture unexpected trends of shifting directions (Freeman et al., 2018; Lenoir et al., 2010).

Climate and climate warming operate on the macroscale and thus form the species-extrinsic influencing factors on this level (see also section 4.3.3 climate expression across scales).

With respect to within-macroscale interactions, some species have the potential to provoke a macroscale feedback loop, so that vegetation cover and climate are affected by species 
Table 4.1. - Description of the relevant scales, ecological units and the respective basic entities and units. Grain information is very specific for the focal organism. Response time can serve as basis for temporal grain. Spatial and temporal extent and grain in parentheses are intended as advice, but may differ between species, e.g. an endemic species requires a different spatial extent and grain compared to a wide-spread generalist or a long-living species a different temporal extent compared to an annual species.

(a) Basic description

\begin{tabular}{lllll}
\hline Scale & Level & Basic entity & Spatial extent & Temporal extent (Grain) \\
\hline Macroscale & Species & Metapopulations & Range & Decades to centuries (year) \\
Mesoscale & Metapopulation & Local populations & Region & Several generations (cohort) \\
Microscale & Community & $\begin{array}{l}\text { Individuals of the } \\
\text { community }\end{array}$ & Locality & Reproductive cycle (day) \\
& & comay & \\
\hline
\end{tabular}

(b) Processes, drivers and output

\begin{tabular}{|c|c|c|c|}
\hline Scale & Major pattern or process & $\begin{array}{l}\text { Driving forces and } \\
\text { influencing factors }\end{array}$ & Information output \\
\hline Macroscale & Range dynamics & $\begin{array}{l}\text { Climate } \\
\text { Evolution }\end{array}$ & $\begin{array}{l}\text { Range border dynamics } \\
\text { Abundance shift }\end{array}$ \\
\hline Mesoscale & $\begin{array}{l}\text { Population growth } \\
\text { Dispersal }\end{array}$ & $\begin{array}{l}\text { Landscape connectivity } \\
\text { Stability of local } \\
\text { populations }\end{array}$ & $\begin{array}{l}\text { Presence/Absence } \\
\text { Local abundance } \\
\text { Colonialization time } \\
\text { Dispersal and gene flow }\end{array}$ \\
\hline Microscale & $\begin{array}{l}\text { Individual growth } \\
\text { Reproduction }\end{array}$ & $\begin{array}{l}\text { Biotic interactions } \\
\text { Resource availability }\end{array}$ & $\begin{array}{l}\text { Individual fitness } \\
\text { Reproductive output } \\
\text { Local community dynam- } \\
\text { ics }\end{array}$ \\
\hline
\end{tabular}

range dynamics. For example, the shift of tree lines towards the poles or upslope on mountainous landscapes (e.g. Gehrig-Fasel et al., 2007; Grace et al., 2002; Scott et al., 1997) might impact regional climate through significant changes of evapotranspiration rates.

Increasing extinction rate can lead to loss of vegetation cover and again result in changes of climatic conditions. In summary, this feedback loop can occur wherever range dynamics patterns of one species or several species result in large-area transformation of an ecosystem and ecosystem functions (Beerling et al., 1998; ?). In fig. 4.1, the dashed arrow from range dynamics to climate visualizes the possible impact of ecosystem change caused by range dynamics on climate dynamics. 


\section{Mesoscale: Metapopulation level}

The mesoscale serves as linking level between the species' full-range level at the macroscale and the microscale community level (Cheruvelil et al., 2017). In our framework, this level reflects a metapopulation on a regional scale, i.e. comprising several local populations. The most important processes here are positive or negative population growth at inhabited sites, extinction events, colonialization of novel sites and gene flow between the populations (fig. 4.1 and table 4.1).

The mesoscale should account for the size of the constituent populations of the entire metapopulation. Knowing the size of populations holds important information for prediction of metapopulation dynamics and in the end for the prediction of species current and future distribution (Duputié et al., 2014; Ovaskainen and Hanski, 2001). Providing information on population sizes throughout the entire range allows for capturing distribution shifts within the observed range and gives further insights into species range and distribution dynamics (Breshears et al., 2008; Sagarin et al., 2006). Observable patterns may for example be a spatial shift of greatest abundance, which may be related to a geographical shift of the bioclimatic optimum. Such a shift might inform on imminent sudden colonialization events as a result of a mass-effect, where the species was unsuccessful before (Alexander et al., 2016; Jackson and Sax, 2010).

A global decrease of abundance is also a strong indicator of the low ability of a species to adapt to novel conditions, in some cases also coupled with the inability to escape from increasingly hostile conditions, e.g. mountainous species that reach the summit (Pertoldi and Bach, 2007). This is a significant advantage for long-term prediction of impact of climate warming on species as compared to pure presence/absence models.

On this scale, landscape configuration is the explicitly modelled species-extrinsic factor. Under the term landscape configuration, we gather two aspects. First, landscape configuration can facilitate or impede connectivity between localities and exert a significant impact on metapopulation dynamics (Thompson et al., 2017). For example, an extended east-west mountain range such as the Alps may pose an insuperable barrier to south-north dispersal of species (Engler and Guisan, 2009). Well connected localities allow for gene flow between populations and thereby increase their adaptation potential (Cobben et al., 2012; Jump and Penuelas, 2005). For colonialization attempts reachability of suitable habitats is 
crucial, either directly or through availability of "stepping stone" habitats (Hannah et al., 2014; Saura et al., 2014). Connectivity between habitats gains more and more importance in the age of anthropogenic fragmentation of the landscape (Bennie et al., 2013; Cobben et al., 2012; Marini et al., 2012). Besides connectivity of habitat patches, size of habitat patches plays a crucial role for non-generalist species (Bender et al., 1998; Holyoak and Heath, 2016). Hence, landscape configuration in terms of topography and fragmentation play a crucial role to determine the degree of connectedness between localities and suitable habitat patches. A derived connectivity map will be very species-specific, depending on its dispersal capability, e.g. dispersal distance and percentage of long-distance dispersal (Saura et al., 2014; Soons and Ozinga, 2005).

With respect to within-mesoscale interactions, regional landscape configuration can influence local and regional climate massively (Gettelman and Rood, 2016). For example, a mountain range such as the Harz hill range in Northern Germany is characterized by a wetter precipitation regime ( $\sim 1000 \mathrm{~mm}$ pa on the mountain tops) than the surrounding low lands $(\sim 600 \mathrm{~mm} \mathrm{pa})$ and additionally causes a "dry shade" $(\sim 500 \mathrm{~mm}$ pa $)$ on its lee side (Hattermann et al., 2011).

\section{Microscale: Community level}

The basic component on the microscale is the individual plant, embedded in a surrounding community. The main output information from this level is local population growth and amount of seeds dispersed outside of the community (table 4.1.

Individual performance of the plant, i.e. growth and survival, and reproductive output are determined by environmental conditions, i.e. habitat suitability, and biotic interactions.

Here, habitat suitability reflects abiotic conditions. This includes climatic factors such as length of vegetation period and surface temperature or microclimate (Rosenberg et al., 1983). Microclimate is partially controlled by local conditions. For example, mountain slopes can show largely different microclimates, depending on their north- or south orientation (Austin and Niel, 2011; Karrasch, 1973). Water bodies can buffer temperature variation during a day or even a year (Assouline and Mahrer, 1996, see also section 4.3.3). The steering effect of such micro-scale factors is also considered in modern urban planning (Buyadi et al., 2013). The type of ecosystem also has an influence on microclimate, e.g. 
a forest as compared to open grassland. In this context, the influence of ecosystem type on habitat suitability is not to be directly seen as biotic interaction, because it does not change over the considered time frame at the microscale.

Additionally, habitat suitability encompasses sufficient provision of necessary resources, i.e. water, light and nutrients, through soil type or primary raw material. The pool of resources directly impacts survival of plants and thus determines carrying capacity of a patch.

While provision of resources is a feature of the abiotic environment, availability of the provided resources for the individual plant additionally depends on immediate biotic interactions. Resource availability can be influenced positively by mutualists, e.g. mycorrhizal fungi (Johnson et al., 2010) and microorganisms (Compant et al., 2010), or facilitating interactions with other plants (Chu et al., 2008; Hauggaard-Nielsen and Jensen, 2005). On the negative end of the interaction spectrum is competition with neighboring plants for the same limiting resources. Provided a finite resource pool, any more successful competitor can pre-empt resources in shared space, often implemented as overlapping zones-of-influence (ZOI) of plants (Radny and Meyer, 2018; Schneider et al., 2006; Schwinning and Weiner, 1998). These resources then are missing for the focal plant individuals' own growth and metabolism, potentially resulting in death or decreased reproductive output (Brown et al., 2004; Radny et al., 2018).

To cope with competitive interactions, plant species can draw on sets of species-specific traits (Goldberg and Landa, 1991; Goldberg, 1996), including for example strategies to outcompete neighboring individuals through pre-empting resources (Bauer et al., 2004; Berger et al., 2006; Schwinning and Weiner, 1998) or weakening neighboring plants through allelopathy (Bais et al., 2003; Hierro and Callaway, 2003), as well as developing tolerance mechanisms (Radny et al., 2018, see also chapter 2. These different strategies multiply to a very complex network of potential expressions of competitive interactions, depending on the identity of the interaction partners. We expect species identity of interacting individuals to have a major impact on form and strength of competitive interactions for individuals of the focal plant species.

Besides these resource-related biotic interactions, enemy attack is another biotic interaction that can have a considerable impact on individual performance. The immediate feeding damage can result in a decreased fitness of the plant individual, e.g. by reducing leaf 
tissue or fine roots and thereby decreased capacity of capturing resources. Compensatory growth (Lu and Ding, 2012) up to a level of overcompensation (Belsky et al., 1993), and increased resource allocation to defense mechanisms (War et al., 2012) are potential secondary effects of enemy attack. Indirect defense mechanisms, e.g. attraction of enemies (Kessler and Baldwin, 2001) or parasitoids (Harvey and Fortuna, 2012; Loon et al., 2000), highlight the importance of multitrophic interactions (dotted arrow between enemies and mutualists in fig. 4.1). Presence or absence of defense-related mutualistic species as well as pollinators is also an important impact factor for individual performance. We expect that abundance of specialized enemies and mutualists depend on host species density, indicated with a double-headed arrow in fig. 4.1.

Altogether, the described abiotic factors and biotic interactions determine individual survival, performance (growth) and reproductive output in form of seeds (Radny et al., 2018, see chapter 2). Reproductive output can result in same-patch recruitment when seeds remain within the parental patch. Depending on dispersal strategy of species, a certain percentage of all produced seeds will be dispersed away from the parental patch, e.g. through wind or animal dispersal vectors (Nathan, 2006). Dispersal away from as well as into the focal patch plays an important role for local population growth (Nathan and Muller-Landau, 2000; Soons and Ozinga, 2005). For example, a highly dispersive species might have a very low local reproduction rate, but will also receive many seeds from interconnected populations. Each local population provides information about the amount of propagules ready for migration as result of performance of the individuals of the focal species. Yet, realization of actual migration rates is influenced by landscape configuration on the mesoscale. Probability of colonialization events in surrounding patches thus is determined by production of dispersing seeds and regional landscape configuration in concert.

\subsubsection{Cross-scale emergences and interactions}

\section{Cross-scale emergences}

Cross-scale emergence is the accumulated interaction of components on a lower level, which produces patterns and processes on a higher level (Heffernan et al., 2014). Note 
that emergence is also a core concept in individual-based modelling, where populationor community-level dynamics emerge from interactions between individual organisms. In fig. 4.1, blue boxes highlight the most important cross-scale emergences in this model concept, i.e. local population dynamics as a result of local biotic interactions and microclimate, to metapopulation dynamics and eventually species range dynamics. In fact, overall population dynamics throughout the range and in novel patches is the most important emergence from the model, directly related to the guiding question.

A second type of emerging pattern is adaptation as evolutionary process. By definition, evolution is a long-term process which affects the entire species. In fact, where not the entire species is affected, evolution results in the formation of new species (Darwin, 1859; Schluter, 2001). Hence, in this framework evolution operates on the macroscale, yet it is driven by adaptation of local populations, or of individuals in case of long-lived species such as trees. Here, edge-populations might play a crucial role for the adaptation potential of the species. Under the assumption of harsher, i.e. less suitable, environmental conditions on the range edges of a species than compared to the center of the range, edge populations are expected to exhibit greater adaptation potential than center populations (Rehm et al., 2015). Gene flow between central populations and edge populations could be of crucial importance to allow for adaptation to changing environmental conditions.

A third type of pattern emergence is related to the concept of local generation, which is a variable inherent to the microscale and which affects the self-sustainability of a species at the macroscale. For the scope of this concept, we define local generation as the continuous number of cohorts in any one local habitat which to a larger part consists of offspring from plant individuals of the same patch. That is seeds which have not been dispersed from another population. We consider this an important variable to determine self-sustainability of a population. When self-sustainability is not given, the given habitat should not be considered as part of the species' range. Thus, local generation on the microscale produces pattern emergence on the macroscale.

\section{Cross-scale interactions}

Climate expression through scales Climate warming is a global phenomenon. Yet, it impacts ecosystems and communities through all scales from global to local with differences 
in the kinds and strengths of impacts on each scale.

Per definition, climate is the long-term average of weather conditions in an area and climate change arises as differences from these long-term statistics (WMO, 1966). Climate warming is the increase of average surface temperature and can cause a cascade of further climate changes, e.g. persistence of atmospheric conditions such as during the summer of 2018 or longer vegetation periods due to earlier spring events.

Within this framework, we suggest to consider climate on a macroscale as climate class based on the Köppen-Geiger classification (Beck et al., 2018; Köppen, 1884; Peel et al., 2007, see fig. 4.3). This allows for a low-effort large-scale classification of a geographical area as potential part of a novel range of a plant species, considering air temperature, precipitation through seasons, and threshold values. The consideration of seasonality is especially important for correct identification of species' niches and transferability to novel localities (Fernández et al., 2012).

While the original maps of the Köppen-Geiger classification are relatively coarse, modern availability of high-resolution climate datasets would allow for much finer resolution, reported to $0.1^{\circ}$ and even finer $0.0083^{\circ}$ (Beck et al., 2018). These resolutions would be sufficiently fine to be applied on a mesoscale. However, the Köppen-Geiger classification system, expressing temperature and precipitation variables, can be understood as a pattern emerging from the interaction between components of the climate system, i.e. atmosphere, water and terrestrial components, for example topography, surface and vegetation cover (Franklin et al., 2013; Gettelman and Rood, 2016). In the context of range dynamics and potential changes in ecosystem structure, implementation of regional climate as function of landscape, vegetation and atmosphere is recommended to capture same-scale feedbacks. Therefore, finer resolutions are not necessary for the Köppen-Geiger classification system to perform well.

On the microscale, climate affects habitat suitability as general climatic conditions that constrain the local weather, directly inherited from the mesoscale. More directly, survival and performance of plant individuals are affected by short-term weather extremes. Here, short-term applies for events on an hourly timescale, such as storms or intense rainfall, but also to events with durations of several weeks, such as the drought periods in Europe in 2003 and 2018. Several, although not all, extreme weather events are expected to increase 


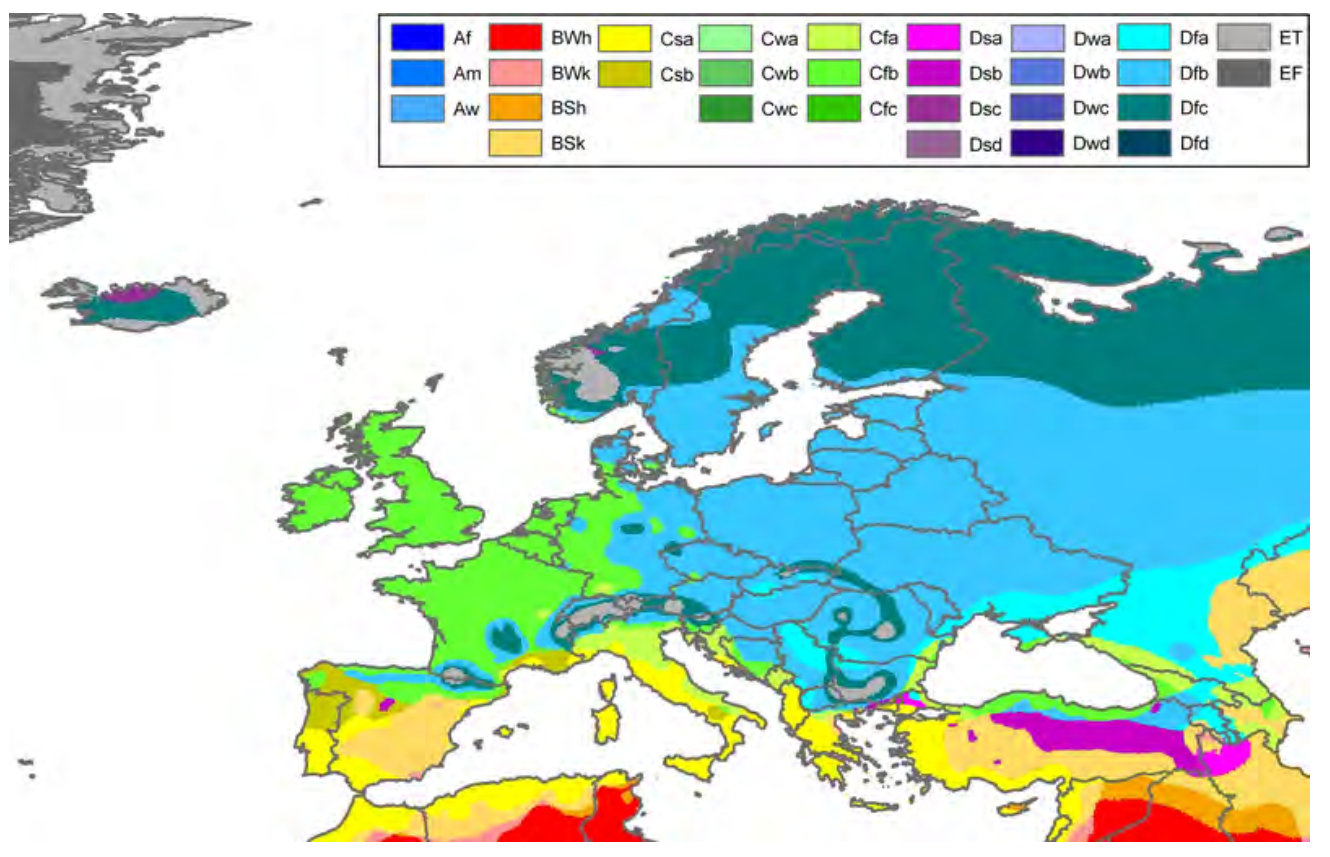

Figure 4.3. - Köppen-Geiger climate type map of Europe at relatively coarse grain. The prevailing climate types in Central Europe are temperate continental climate/humid continental climate (Dfb), temperate oceanic climate (Cfb). The Harz mountain range clearly stands out as cool continental climate type (DfC). Original map: (C) Peel et al. (2007) / CC BY-NC-SA 2.5.

in frequency under current climate warming (Ummenhofer and Meehl, 2017). Thus, it should be carefully considered whether or not the probability of such extreme events is coupled to climate warming when implementing them into a model.

Climate as influencing factor on biotic interactions We have described how biotic interactions can hamper or improve individual performance in already inhabited as well as novel habitats section 4.3.2. While these biotic interactions can directly be related to performance and survival of a focal individual, changes in climatic conditions can influence these interactions and therefore exert an indirect impact on individual plant performance at the microscale (Thomas, 2010). For example, increasingly mild winters may lead to higher winter survival of herbivorous insects (Bale et al., 2002; Kreyling, 2010), thereby increasing enemy pressure and related plant mortality.

Climate warming may also weaken plant competitors which formerly inhibited colonialization of the focal species in a novel community (Alexander et al., 2016; Bauer, 2012).

Through different response of organisms to climate warming, altered bioclimatic conditions have the potential to disrupt local interaction networks, plant-enemy interactions as well 
as plant-mutualist interactions (Caddy-Retalic et al., 2018; Harvey et al., 2010; Van der Putten, 2012). For example, different dispersal capacities can result in spatial mismatch of a mobile insect and its sessile host plant (Schweiger et al., 2008). The other way round, a plant species with high dispersal capacity may escape slower enemies (Lakeman-Fraser and Ewers, 2013), but also lose soil-borne mutualistic species, e.g. microbes that reduce allelopathic chemicals released by neighboring species (Ehlers, 2011). Differential response to earlier spring can result in temporal mismatch of plants and pollinators (Kreyling, 2010).

The network of biotic interactions might become even more fragile when considering not only first-order trophic interactions, but multitrophic interactions and hence a multi-species system (Morriën et al., 2011). Nevertheless, future formation of such non-analogous communities which greatly differ from the original communities will likely pose a significant source of uncertainty to modelling of range dynamics and species' response to climate warming (Williams and Jackson, 2007).

\subsubsection{Uncertainties}

In general, any model is vulnerable to two kinds of uncertainties: (1) external uncertainties, i.e. future development of surrounding conditions, and (2) internal uncertainties which arise from the structure of the model as such.

\section{Climate warming rate}

A major external uncertainty of modelling climate warming-induced range dynamics of species is the development of climate warming itself. Under the assumption that current climate warming is strongly driven by anthropogenic drivers, e.g. emission of greenhouse gases (IPCC, 2014), political decisions and societal developments will eventually determine where the journey will lead to. Such uncertainties can be addressed by running an implemented model under different warming scenarios. We expect to include a reasonable proxy for regional climate development through implementation of mesoscale climate under consideration of vegetation as part of the biosphere and landscape configuration as part of the hydrosphere and the lithosphere (Gettelman and Rood, 2016). However, the closer the potential feedback loops of those components approach a threshold with a tipping 
point, the greater is the uncertainty of the outcome (Groffman et al., 2006; Peters et al., 2007). Hence, the point of state change of a region, e.g. in terms of Köppen-Geiger class, should be carefully evaluated to achieve a reasonable estimate of potential abrupt changes (Groffman et al., 2006; Ratajczak et al., 2018).

\section{Landscape structure}

Landscape fragmentation and habitat loss have been identified as critical factors for species responses to climate warming (see section 4.3.2) and can cause external uncertainty in range models. Anthropogenic land use is a main reason for increasingly fragmented landscapes, provoking habitat degradation or habitat loss (Cheptou et al., 2017), and is a matter of sociopolitical decisions and developments. Heffernan et al. (2014) recommend inclusion of the sociopolitical axis into macrosystems ecology frameworks, especially when assuming a strong and ongoing anthropogenic impact on climate warming rate. For our framework, we primarily focus on ecological aspects of range dynamics to evaluate the ecological potential of species to escape from negative impacts of climate warming. If the framework is implemented to inform policy makers and conservationists, it is highly recommended to explicitly consider the effect of anthropogenic land use on landscape fragmentation to derive reliable estimates (Bonebrake et al., 2018).

\section{Changing and novel interactions}

Simplification can lead to internal uncertainties. Representation of complex systems in simulations or other modelling techniques always requires a certain degree of simplification (Domingos, 1999). This simplification leads to uncertainties in the model, for example when important driving factors were not considered, just because their actual relevance for the entire system was underestimated or unknown. Especially in multitrophic interaction webs, crucial interactions may be overlooked. One popular example for such an underestimation was the indirect impact of the absence of wolves on the vegetation in the Yellowstone National Park. Reintroduction of this large predator had significant impacts on population size of browsers, such as elk, and improved development of the forest ranges (Smith et al., 2003).

With respect to range dynamics, a similar disruption of interaction webs may appear when 
mutualistic or antagonistic species cannot keep pace with a range shifting species (Van der Putten, 2012). This could be of greater importance the more specialist interactions a focal species experiences within its current range (Gilman et al., 2010). This might require an extension of the framework to a multispecies approach if certain interactions appear to be crucial for colonialization and survival of a focal species. Additional to the loss of established interaction partners, we expect encounters of novel interaction partners during colonialization events of the focal species or by movement dynamics of these novel partners. Such new interactions can lead to unexpected effects (Williams and Jackson, 2007). For example, if a novel plant attracts beneficial soil-biota while simultaneously being released from enemies, this can result in an unexpectedly high performance of the individual (Van Grunsven et al., 2010). Such overly well-performing species bear the threat of becoming invasive, in the sense of destabilizing local community, and potentially decrease local biodiversity. This might be even more pronounced when climate warming is favoring the novel species, while resident species suffer disadvantages from the altered bioclimatic conditions (Bauer, 2012).

It will be difficult to assess the outcome of novel interactions between species, especially since either interaction partner may be affected by changing climatic conditions in a different way. A solution may be to approximate novel interactions based on a comparison of ecological types or trait profiles of the interacting species (Bruelheide et al., 2018; Soliveres et al., 2014).

\section{Extent of the modelled range}

Size of modelled range is related to further internal uncertainties. To capture the full extent of range dynamics (fig. 4.2), the modelled area needs to comprise the focal species' entire current range at initialization as well as a reasonable extended potentially inhabitable zone. This extended zone does not have a record of established populations on initialization of the model, but lies in a distance which could be reached within the time of observation. The appropriate spatial extent then depends on the temporal extent modelled and expected dispersal velocity as function of dispersal capacity of the species, seed production of the local populations and chance for long-distance dispersal events, as well as landscape configuration and connectivity of patches. Uncertainties in dispersal and environmental parameters may 
necessitate a fairly large potentially inhabitable zone. For example, rapid and accelerating climate warming could result in future inhabitability of localities far away from the initial range borders, while rapid evolution might select for increasing dispersal ability of species ( e.g. Boeye et al., 2013; Cheptou et al., 2008, but see Phillips, 2012).

Upon initialization of the respective model, an extensive potentially inhabitable zone might result in a very high computational load generated by patches without population records. In order to relax computational load, an adaptive mesh could be applied, where coarse grain is adapted to finer grain upon abundance records of the respective species (Lischke et al., 2007; Wiegand et al., 2008).

\section{Parametrization}

Parameterization poses another serious challenge and source of internal uncertainty for such a model. In this concept, we have included many parameters and influencing factors, which we expect to be of crucial importance. Even when acknowledging long-term field experiments, such as the "Jena experiment" (Roscher et al., 2004; Weigelt et al., 2010), and the vast data pool they produced, there will hardly be the chance to capture all parameters in a single study. More commonly, parametrization will necessarily be fed from many different sources, such as scientific databases. This often implies different sampling methods, surrounding conditions, and sample scale. Approximation of derived data for the respective modelling goal will pose another severe source of uncertainty.

\subsection{Discussion}

Macrosystems ecology provides a theoretical framework to model complex ecological systems through organizational levels that operate on different spatial and temporal scales and are interconnected through bottom-up cross-scale emergence and top-down cross-scale interactions. Using this framework, we have developed an individual-based model concept to simulate climate warming-induced range dynamics of plant species, accounting for macroscale, mesoscale and microscale patterns and processes (fig. 4.1). The overarching question to be answered using this concept is which pattern of range dynamics will be observed for a plant species under current climate warming (fig. 4.2). 
Realization of this pattern depends on three basic features: (a) future habitat suitability of localities within and beyond a species' current range, (b) reachability of these habitats at a similar pace as the potentially rapid progress of climate warming, and (c) ability to colonize new locations. We have highlighted drivers and processes that influence realization of the range dynamics pattern and grouped them in three levels on corresponding spatial scales.

Implementation of a model following this framework will not be trivial. Complications arise from (a) potential pitfalls from scaling, (b) dealing with uncertainties, and (c) model complexity.

\subsubsection{Scaling and associated sources of bias}

The processes underlying the emerging range dynamics patterns operate at different spatial and temporal scales, but are connected through cross-scale emergence and interactions. Implementation of a full model according to the proposed framework will require scaling-up from finer to coarser grain, i.e. from lower to higher level. This goes along with aggregation of information from the lower levels and a potential loss of fine-grained information on heterogeneity (Bocedi et al., 2012). Severity of this bias is likely to increase with increasing spatial or temporal variability.

Heterogeneity is an important feature of macrosystems with potentially significant impacts on modelling results (Heffernan et al., 2014). For example, in our framework, landscape, or more precisely landscape configuration, is acknowledged as determining factor for realization of dispersal between populations and into novel habitats, as well as for regional climate. Additionally, on the microscale landscape configuration interacts with local microclimate, potentially creating holdouts and stepping stones for populations. In a rapidly changing environment, these refugia can crucially contribute to species' persistence and realization of range border shift (Hannah et al., 2014; Saura et al., 2013).

Envisaging the impact of fine-grain landscape features, aggregation technique of landscape information has to be chosen carefully when applying a model for decision making as it may underestimate a species' capacity to track suitable climatic conditions in the future. On the same track, homogenization of a simulated landscape can also overestimate the availability of potential habitat, leading to false-optimistic assumptions on population stability (Franklin 
et al., 2013). Such an overestimation of population stability can also arise where spatiallyexplicit processes are blurred out during scaling-up. Bocedi et al. (2012) highlighted an overestimation of local carrying capacity when information about local density-dependent population growth is lost through aggregation of neighborhood patches. Additionally, they demonstrate how dispersal distance and hence the capability of species to track climate change is overestimated with increasingly coarse resolution of the landscape.

With respect to macrosystems ecology, Cheruvelil et al. (2017) present an interesting approach to cluster landscape units with respect to heterogeneity: spatially constrained spectral clustering where regions are clustered by homogeneity and contiguity, with user-defined sensitivity and selection variables (Cheruvelil et al., 2017; Yuan et al., 2015). The authors achieved reasonable clusters to capture variation of water body characteristics on a regional scale. While published applications in population dynamics or range dynamics are lacking to date, we consider this a worthwhile approach to follow. Potential clustering variables within the scope of our guiding question could be similarity of community functional trait composition, microclimatic conditions and/or local population densities. Together with alternative dispersal representations, e.g. diffusion models (Bocedi et al., 2012 and references therein; Hefley et al., 2017), current biases might be reduced to an acceptable level for application-oriented predictions.

\subsubsection{Dealing with uncertainty}

Non-linear behavior and cross-scale interactions and emergence between higher and lower levels pose an additional challenge to model implementation. Several known and unknown but likely interactions and feedback loops exist between levels of ecological systems. In fact, ranges of species and the distribution patterns within species ranges are driven by many interacting processes on different scales (Sagarin et al., 2006). The interconnections between the scales are likely to expose a non-linear or threshold behavior and hence put the a linear scaling-up procedure at question (Peters et al., 2004). Additionally, when extrapolating the model to future conditions, there is an increasing chance of "ecological surprises", arising from non-analogous climates as well as novel interactions (Alexander et al., 2016; Bauer, 2012; Williams and Jackson, 2007). 
Altogether, this results in a high complexity as well as a high degree of uncertainty to an applied model. Bayesian modelling approaches can be used to deal with uncertainties in parameterization, heterogeneous data sources and non-linear behavior of the system (Cressie et al., 2009; Levy et al., 2014; Munch et al., 2017). In fact, spatio-temporal Bayesian models on complex interactions (Fraterrigo et al., 2014; Schneider et al., 2006), on the prediction of species distribution and abundance (Ramsey et al., 2015; Zillio and $\mathrm{He}, 2010$ ), and on range dynamics of plant and animal species (Hooten and Wikle, 2008; Wikle and Hooten, 2006) have been around for some time already. Usage of Bayesian approaches is recommended repeatedly in the context of macrosystems ecology and crossscale interactions (Levy et al., 2014; Soranno et al., 2014). For example, integrated Bayesian models can outperform more classical approaches when it comes to uncertainties through cross-scale interactions and data from heterogeneous sources and scales (Talluto et al., 2016).

Overall, it is important to deal transparently with uncertainties in the model building process and model results, because this will contribute to more acceptance of model predictions by decision makers.

\subsubsection{Complexity of the model}

As with any other model, the basic question is at which point to apply "Occams Razor" (Domingos, 1999), i.e. which aspects to include and which to leave out, to determine the optimum level of complexity of the model. We have highlighted those aspects that we consider as most important for our guiding question on the patterns of range dynamics of species under future climate conditions with regard to (a) ecological requirements (future habitat suitability), (b) dispersal (ability to track climate changes) and (c) local population processes (local persistence and establishment success). Surely, there will be processes we did not explicitly consider here, such as implementation of sociopolitical aspects. These aspects will be beneficial to include once the guiding question of the model leaves the realms of basic ecology towards a more applied scope.

Upon implementation of a model following this framework, some interactions and processes we highlighted might be of greater or lesser importance, depending on the focal species. 
However, we advocate to withstand the temptation to rule out too many factors. Several models have shown a significant increase of accuracy when considering additional factors, such as biotic interactions (Staniczenko et al., 2017) or physiology (Talluto et al., 2016). However, over-parametrization of models may result in inherent uncertainty just as underparametrization can result in decreased realism (Singer et al., 2016), as it has been argued for bioclimatic envelope models (Pearson and Dawson, 2003).

Hence, the final choice of relevant parameters is far from trivial and should be carefully evaluated under consideration of the intended focal species' ecological profile.

At the microscale, we expect the highest degree of complexity. On the one hand, this complexity emerges from the involvement of entities on different trophic levels, i.e. other plants, but also insect enemies and mutualists, and where necessary their enemies. On the other hand, individual variability may play an important role, for example when competitive interactions depend on biomass (Radny et al., 2018). At this point, adopting an individual-based approach might be the adequate solution, as individual-based models account for individual characteristics and a multitude of interactions between the involved agents (Grimm and Railsback, 2005). In line with the macrosystems ecology framework of the proposed model structure, individual-based models work with emergence as a principle, contrary to purely mathematical or physical models (DeAngelis and Grimm, 2014). The combination of system-dynamics models with individual-based models is on the rise and provides a highly promising tool for complex ecological systems (Singer et al., 2016; Vincenot et al., 2011). Such hybrid-models have also been used in modelling socio-ecological interactions (Martin and Schlüter, 2015), making them even more attractive in a macrosystems ecology framework.

Of course, the complexity of a model such as the proposed one will come at a cost of either structural realism or reliability due to knowledge gaps (Singer et al., 2016). However, simple models, which leave out important interactions, may be easier to implement and understand, but will not necessarily yield the desired answers (Evans et al., 2013). We have identified several processes at different scales and interactions across scales that we expect to be crucial for realistic estimations of range dynamics. We are very positive that large data repositories and advances in big data handling (Farley et al., 2018; Kress, 2019) will make it possible that such a model will be implemented in the near future. 


\section{Concluding discussion}

This thesis had the aim to investigate mechanisms of plant range dynamics under climate warming. Current climate warming has many direct and indirect impacts on the performance of plants, which can lead to shifts of range and distribution patterns of species (Parmesan and Hanley, 2015; Parmesan and Yohe, 2003; see also chapter 1). Realized range shifts emerge from dispersal to, and establishment in novel habitats. These stages have very different underlying processes and hence, are likely to require different abilities (Estrada et al., 2016). For my study, I related the performance of individuals to their ability to compete with resident neighbours (chapters 2 and 3 ) and defend against herbivores (chapter 3). To assess large-scale range dynamics of species, local population dynamics have to be extended by dispersal capacity (chapter 4). Competitive, defensive and dispersal capacity are expressed by corresponding traits and give rise to the ecological profile of a species (table 5.1 and fig. 5.1). Such a profile may be used to predict species' range dynamics under consideration of local biotic interactions and through different stages of range shift. For novel plant species from other continents, the combination of traits explained invasive success better than single traits (Küster et al., 2008). Furthermore, embedding trait combinations in a context of locally relevant biotic interactions (e.g. Adler et al., 2012) may further increase the predictive power for local establishment success of species. Trait-based approaches have gained increasing attention to analyse and predict range shifts (e.g. Butt and Gallagher, 2018; Estrada et al., 2018; Tremlová and Münzbergová, 2007). The growing body of literature underlines the relevance of trait-mediated spread and establishment success for explaining range dynamics, although a unifying scheme has not yet emerged (MacLean and Beissinger, 2017). With my thesis, I contributed an approach to understand the mechanisms that drive establishment success of novel plants under different degrees of biotic pressures based on the corresponding competition and defence traits, and highlighted which factors gain importance in the dispersal stage. 


\section{Concluding discussion}

Table 5.1. - List of possible trait combinations of competitive, defensive and dispersal ability. These combinations build the ecological trait profiles mentioned in this thesis. High trait values are indicated with " + ", low trait values are indicated with "-". Competitive ability is related to seed size in the studies of this thesis (chapter 1 and 2). Defensive ability can be related to mechanical barriers or toxic compounds and is implemented as probability to repel insect herbivores (chapter 2). Dispersal ability can be expressed by the shape of the dispersal kernel and should reflect the ability to reach novel suitable habitats (see concept in chapter 4). In full factorial combinations, nine ecological profiles would be possible. The first row lists the notation of the nine trait profiles.

\begin{tabular}{lcccccccc}
\hline & HiAll & HiComp & HiDef & HiDisp & LoComp & LoDef & LoDisp & LoAll \\
Competition & + & + & - & - & - & + & + & - \\
Defense & + & - & + & - & + & - & + & - \\
Dispersal & + & - & - & + & + & + & - & - \\
\hline
\end{tabular}

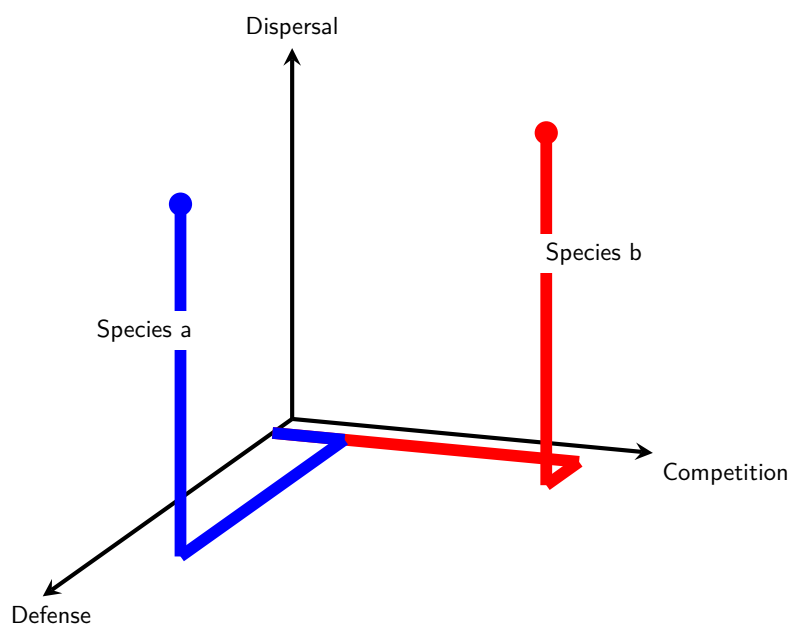

Figure 5.1. - Schematic profile examples. Axis origin is weak expression of traits, and each axis describes increasing strong expression of the respective traits. In this scheme, Species a is characterized by low competitive strength (value on the $y$-axis), and high defense and dispersal trait values (values on $x$ - and $z$-axis). Species $b$ is characterized by high competitive strength and dispersal capacity (values on $y$ - and $z$-axis) and weak defense trait value ( $x$-axis). 
In the following, I briefly synthesize how competitive ability and interactions influenced plant performance in the greenhouse experiment (chapter 2) and in the local-scale model (chapter 3) and how the results complement each other. Based on these insights, I propose to account for more complex interactions in plant communities on the local scale (chapter 4), climate-warming driven changes in interactions with the resident community (chapters 1 and 4), and more options of modelling techniques (chapter 4).

In the greenhouse experiment (chapter 2) and the subsequently developed individual-based model (chapter 3) I focused on early local establishment. The greenhouse experiment confirmed that seed size is a reliable predictor of adult biomass and hence can be used as an easily accessible proxy trait for establishment experiments (see also fig. 2.5). According to established competition models (e.g. Lin et al., 2012; Weiner and Damgaard, 2006), having a higher biomass should result in a competitive advantage. This was the case for the individual-based model where strong competitors had a massive advantage in performance and survival as compared to weak competitors (chapter 3). An increase of competitive asymmetry towards more pronounced size-symmetric resource competition further accentuated this effect. Replacement of weaker competitors with stronger competitors has been observed in field observations of the consequences of the introduction of novel plants (Alexander et al., 2015; Morriën et al., 2010) as well as in established communities, when climate warming favoured one species over others (Ma et al., 2017).

However, drawing a competitive advantage from having a greater biomass than neighbouring individuals might be only one, rather intuitive side of the coin. While drop-out rates in the greenhouse experiment were close to none, survival was low in some cases in the model. Here, the plant had to capture a minimum amount of resources relative to its size to maintain its metabolic rate (Lin et al., 2012; Schmitz, 2000). During the experiment, mortality of single individuals seemed to have no relationship to treatment or species identity. This emphasized the potential importance of including further types of interactions, e.g. facilitative interactions (Chu et al., 2008; Lin et al., 2012), as well as differentiation of competitive interactions, depending on the respective interaction partners (Radny and Meyer, 2018). In fact, plants may have developed many different strategies to interact with their neighbours (Novoplansky, 2009), which appears a "reasonable" adaptation considering the immobile nature of plants. In chapter 3, I suggested to implement such 


\section{Concluding discussion}

partner-sensitive competitive interactions through variation of the degree of competitive asymmetry. To derive the corresponding interaction parameters either from experiments or from data repositories, it is necessary to identify the most relevant interactions for the system under investigation. This requires also system-specific knowledge about how the system will most likely be affected under climate warming (see examples in chapter 1 ) and how these changes manifest in processes and patterns on local and broader scales (chapter 4).

The complexity of the modelling framework that I propose in chapter 4 to address the plant species responses to climate warming outlined in chapter 1 is likely prone to uncertainties in parametrization. Recent development in modelling techniques, such as highly evolved mechanistic species distribution models may pave the way for more realistic modelling (Cabral et al., 2017; Singer et al., 2016). Probably the most feasible technical solution may be the combination of analytical models with individual-based models. Analytical models are highly suitable to capture processes on broader scales, i.e. the meso- and macroscale or metapopulation and species level, whereas individual-based models are ideal to simulate complex community interactions at the microscale. Individual-based models can explicitly account for biotic interactions including many types of agents and relationships between them (DeAngelis and Grimm, 2014; Grimm and Railsback, 2005) and provide a promising, underexplored basis to be combined with analytical approaches (Travis et al., 2011).

Overall, I think it will be possible to construct an ecological profile from species traits to unravel the mechanisms underlying climate warming-induced range shifts of plants. Recently, the consideration of combinations of traits confirmed an increase of explanatory power of plant establishment and spread as compared to single trait approaches (Estrada et al., 2018; Küster et al., 2008). Ecological trait profiles might gain considerable importance for assessing multispecies dynamics with relative generality (McGill et al., 2006) and at relatively low computing costs (Zakharova et al., 2019), but still producing very realistic patterns (Cabral and Kreft, 2012). In light of an predicted increase in non-analogue communities (Williams and Jackson, 2007) that have little to no similarity to any known community, the generality of trait-based approaches may result in more flexible predictive models compared to species-based approaches as a better preparation for "ecological sur-

prises". However, as my new model concept shows (chapter 4), such an ecological trait 
profile must consider more factors than covered by the empirical and modelling studies of this thesis (chapters 2 and 3 ) to be applicable to a broad set of abiotic and biotic conditions. For example, these factors may include facilitative interactions and dependency on mutualistic interactions (chapter 4), as well as vulnerability to locally changing conditions (chapter 1). This thesis has provided a few puzzle pieces (chapters 1-3) and a sketch of the full picture (chapter 4 ) that is required to predict climate warming-induced range shifts of plant species in multispecies interaction webs under different future scenarios of global climate warming across spatial and temporal scales. 



\section{Literature}

AbdElgawad, H., Farfan-Vignolo, E. R., Vos, D. d., and Asard, H. (2015). Elevated CO2 mitigates drought and temperature-induced oxidative stress differently in grasses and legumes. Plant Science, 231:1-10.

Adler, P. B., Dalgleish, H. J., and Ellner, S. P. (2012). Forecasting plant community impacts of climate variability and change: when do competitive interactions matter? Journal of Ecology, 100(2):478-487.

Agrawal, A. A. and Fishbein, M. (2006). Plant defense syndromes. Ecology, 87(sp7):S132S149.

Aikio, S. (2004). Competitive asymmetry, foraging area size and coexistence of annuals. Oikos, 104(1):51-58.

Alexander, J. M., Diez, J. M., Hart, S. P., and Levine, J. M. (2016). When climate reshuffles competitors: a call for experimental macroecology. Trends in Ecology \& Evolution, 31(11):831-841.

Alexander, J. M., Diez, J. M., and Levine, J. M. (2015). Novel competitors shape species' responses to climate change. Nature, 525(7570):515-518.

Anderegg, W. R. L., Kane, J. M., and Anderegg, L. D. L. (2013). Consequences of widespread tree mortality triggered by drought and temperature stress. Nature Climate Change, 3(1):30-36.

Araújo, M. B. and Pearson, R. G. (2005). Equilibrium of species' distributions with climate. Ecography, 28(5):693-695.

Araújo, M. B. and Peterson, A. T. (2012). Uses and misuses of bioclimatic envelope modeling. Ecology, 93(7):1527-1539.

Ariza, C. and Tielbörger, K. (2012). Biomass explains the intensity of facilitative-not competitive-interactions: three intraspecific tests with annuals. Web Ecology, 12(1):4955 .

Assouline, S. and Mahrer, Y. (1996). Spatial and Temporal Variability in Microclimate and Evaporation over Lake Kinneret: Experimental Evaluation. Journal of Applied Meteorology, 35(7):1076-1084.

Austin, M. P. and Niel, K. P. V. (2011). Improving species distribution models for climate change studies: variable selection and scale. Journal of Biogeography, 38(1):1-8.

Bais, H. P., Vepachedu, R., Gilroy, S., Callaway, R. M., and Vivanco, J. M. (2003). Allelopathy and Exotic Plant Invasion: From Molecules and Genes to Species Interactions. Science, 301(5638):1377-1380. 


\section{LITERATURE}

Bakkenes, M., Alkemade, J. R. M., Ihle, F., Leemans, R., and Latour, J. B. (2002). Assessing effects of forecasted climate change on the diversity and distribution of European higher plants for 2050. Global Change Biology, 8(4):390-407.

Bale, J. S., Masters, G. J., Hodkinson, I. D., Awmack, C., Bezemer, T. M., Brown, V. K., Butterfield, J., Buse, A., Coulson, J. C., Farrar, J., Good, J. E. G., Harrington, R., Hartley, S., Jones, T. H., Lindroth, R. L., Press, M. C., Symrnioudis, I., Watt, A. D., and Whittaker, J. B. (2002). Herbivory in global climate change research: direct effects of rising temperature on insect herbivores. Global Change Biology, 8(1):1-16.

Balling, R. C., Meyer, G. A., and Wells, S. G. (1992). Climate change in Yellowstone National Park: Is the drought-related risk of wildfires increasing? Climatic Change, 22(1):35-45.

Bauer, J. T. (2012). Invasive species: "back-seat drivers" of ecosystem change? Biological Invasions, 14(7):1295-1304.

Bauer, S., Wyszomirski, T., Berger, U., Hildenbrandt, H., and Grimm, V. (2004). Asymmetric competition as a natural outcome of neighbour interactions among plants: results from the field-of-neighbourhood modelling approach. Plant Ecology, 170(1):135-145.

Beck, H. E., Zimmermann, N. E., McVicar, T. R., Vergopolan, N., Berg, A., and Wood, E. F. (2018). Present and future Köppen-Geiger climate classification maps at 1-km resolution. Scientific Data, 5:180214.

Becker-Scarpitta, A., Vissault, S., and Vellend, M. (2019). Four decades of plant community change along a continental gradient of warming. Global Change Biology, 0(0).

Becklin, K. M., Anderson, J. T., Gerhart, L. M., Wadgymar, S. M., Wessinger, C. A., and Ward, J. K. (2016). Examining Plant Physiological Responses to Climate Change through an Evolutionary Lens. Plant Physiology, 172(2):635-649.

Beerling, D. J., Chaloner, W. G., Woodward, F. I., and Hayden, B. P. (1998). Ecosystem feedbacks on climate at the landscape scale. Philosophical Transactions of the Royal Society of London. Series B: Biological Sciences, 353(1365):5-18.

Bellard, C., Bertelsmeier, C., Leadley, P., Thuiller, W., and Courchamp, F. (2012). Impacts of climate change on the future of biodiversity. Ecology Letters, 15(4):365-377.

Belluau, M. and Shipley, B. (2018). Linking hard and soft traits: Physiology, morphology and anatomy interact to determine habitat affinities to soil water availability in herbaceous dicots. PLOS ONE, 13(3):e0193130.

Belsky, A. J., Carson, W. P., Jensen, C. L., and Fox, G. A. (1993). Overcompensation by plants: Herbivore optimization or red herring? Evolutionary Ecology, 7(1):109-121.

Bender, D. J., Contreras, T. A., and Fahrig, L. (1998). Habitat Loss and Population Decline: A Meta-Analysis of the Patch Size Effect. Ecology, 79(2):517-533.

Bennie, J., Hodgson, J. A., Lawson, C. R., Holloway, C. T. R., Roy, D. B., Brereton, T., Thomas, C. D., and Wilson, R. J. (2013). Range expansion through fragmented landscapes under a variable climate. Ecology letters, 16(7):921-9.

Berg, M. P., Kiers, E. T., Driessen, G., Van Der HEIJDEN, M., Kooi, B. W., Kuenen, F., Liefting, M., Verhoef, H. A., and Ellers, J. (2010). Adapt or disperse: understanding species persistence in a changing world. Global Change Biology, 16(2):587-598. 
Berger, A. G., McDonald, A. J., and Riha, S. J. (2006). Scaling plant size to below-ground zone of influence in annuals under contrasting competitive environments. Functional Ecology, 20(5):770-777. WOS:000240288900004.

Berger, U., Piou, C., Schiffers, K., and Grimm, V. (2008). Competition among plants: Concepts, individual-based modelling approaches, and a proposal for a future research strategy. Perspectives in Plant Ecology, Evolution and Systematics, 9(3-4):121-135.

Bindoff, N. L., Stott, P. A., AchutaRao, K. M., Allen, M. R., Gillett, N., Gutzler, D., Hansingo, K., Hegerl, G., Hu, Y., Jain, S., Mokhov, I. I., Overland, J., Perlwitz, J., Sebbari, R., and Zhang, X. (2013). Chapter 10 - Detection and attribution of climate change: From global to regional. In Climate Change 2013: The Physical Science Basis. IPCC Working Group I Contribution to AR5. Cambridge University Press, Cambridge.

Bocedi, G., Pe'er, G., Heikkinen, R. K., Matsinos, Y., and Travis, J. M. J. (2012). Projecting species' range expansion dynamics: sources of systematic biases when scaling up patterns and processes. Methods in Ecology and Evolution, 3(6):1008-1018.

Boeye, J., Travis, J. M. J., Stoks, R., and Bonte, D. (2013). More rapid climate change promotes evolutionary rescue through selection for increased dispersal distance. Evolutionary Applications, 6(2):353-364. WOS:000315184800017.

Bonan, G. B. and Shugart, H. H. (1989). Environmental factors and ecological processes in boreal forests. Annual review of ecology and systematics, 20(1):1-28.

Bonebrake, T. C., Brown, C. J., Bell, J. D., Blanchard, J. L., Chauvenet, A., Champion, C., Chen, I.-C., Clark, T. D., Colwell, R. K., Danielsen, F., Dell, A. I., Donelson, J. M., Evengård, B., Ferrier, S., Frusher, S., Garcia, R. A., Griffis, R. B., Hobday, A. J., Jarzyna, M. A., Lee, E., Lenoir, J., Linnetved, H., Martin, V. Y., McCormack, P. C., McDonald, J., McDonald-Madden, E., Mitchell, N., Mustonen, T., Pandolfi, J. M., Pettorelli, N., Possingham, H., Pulsifer, P., Reynolds, M., Scheffers, B. R., Sorte, C. J. B., Strugnell, J. M., Tuanmu, M.-N., Twiname, S., Vergés, A., Villanueva, C., Wapstra, E., Wernberg, T., and Pecl, G. T. (2018). Managing consequences of climate-driven species redistribution requires integration of ecology, conservation and social science. Biological Reviews, 93(1):284-305.

Bossdorf, O. (2013). Enemy release and evolution of increased competitive ability: at last, a smoking gun! New Phytologist, 198(3):638-640.

Breshears, D. D., Huxman, T. E., Adams, H. D., Zou, C. B., and Davison, J. E. (2008). Vegetation synchronously leans upslope as climate warms. Proceedings of the National Academy of Sciences, 105(33):11591-11592.

Brooker, R. W., Travis, J. M. J., Clark, E. J., and Dytham, C. (2007). Modelling species' range shifts in a changing climate: the impacts of biotic interactions, dispersal distance and the rate of climate change. Journal of theoretical biology, 245(1):59-65.

Brown, J. H. (1984). On the Relationship between Abundance and Distribution of Species. The American Naturalist, 124(2):255-279. 02461.

Brown, J. H., Gillooly, J. F., Allen, A. P., Savage, V. M., and West, G. B. (2004). Toward a Metabolic Theory of Ecology. Ecology, 85(7):1771-1789. 03382.

Bruelheide, H., Dengler, J., Purschke, O., Lenoir, J., Jiménez-Alfaro, B., Hennekens, S. M., Botta-Dukát, Z., Chytrý, M., Field, R., Jansen, F., Kattge, J., Pillar, V. D., Schrodt, F., Mahecha, M. D., Peet, R. K., Sandel, B., van Bodegom, P., Altman, J., Alvarez-Dávila, 
E., Arfin Khan, M. A. S., Attorre, F., Aubin, I., Baraloto, C., Barroso, J. G., Bauters, M., Bergmeier, E., Biurrun, I., Bjorkman, A. D., Blonder, B., Čarni, A., Cayuela, L., Černý, T., Cornelissen, J. H. C., Craven, D., Dainese, M., Derroire, G., De Sanctis, M., Díaz, S., Doležal, J., Farfan-Rios, W., Feldpausch, T. R., Fenton, N. J., Garnier, E., Guerin, G. R., Gutiérrez, A. G., Haider, S., Hattab, T., Henry, G., Hérault, B., Higuchi, P., Hölzel, N., Homeier, J., Jentsch, A., Jürgens, N., Kącki, Z., Karger, D. N., Kessler, M., Kleyer, M., Knollová, I., Korolyuk, A. Y., Kühn, I., Laughlin, D. C., Lens, F., Loos, J., Louault, F., Lyubenova, M. I., Malhi, Y., Marcenò, C., Mencuccini, M., Müller, J. V., Munzinger, J., Myers-Smith, I. H., Neill, D. A., Niinemets, Ü., Orwin, K. H., Ozinga, W. A., Penuelas, J., Pérez-Haase, A., Petř́k, P., Phillips, O. L., Pärtel, M., Reich, P. B., Römermann, C., Rodrigues, A. V., Sabatini, F. M., Sardans, J., Schmidt, M., Seidler, G., Silva Espejo, J. E., Silveira, M., Smyth, A., Sporbert, M., Svenning, J.-C., Tang, Z., Thomas, R., Tsiripidis, I., Vassilev, K., Violle, C., Virtanen, R., Weiher, E., Welk, E., Wesche, K., Winter, M., Wirth, C., and Jandt, U. (2018). Global trait-environment relationships of plant communities. Nature Ecology \& Evolution, 2(12):1906-1917.

Butt, N. and Gallagher, R. (2018). Using species traits to guide conservation actions under climate change. Climatic Change, 151(2):317-332.

Buyadi, S. N. A., Mohd, W. M. N. W., and Misni, A. (2013). Green Spaces Growth Impact on the Urban Microclimate. Procedia - Social and Behavioral Sciences, 105:547-557.

Byers, D. L. (2017). Studying plant-pollinator interactions in a changing climate: A review of approaches1. Applications in Plant Sciences, 5(6).

CABI (2013). Plantwise Knowledge Bank.

Cabral, J. S. and Kreft, H. (2012). Linking ecological niche, community ecology and biogeography: insights from a mechanistic niche model. Journal of Biogeography, 39(12):2212-2224.

Cabral, J. S., Valente, L., and Hartig, F. (2017). Mechanistic simulation models in macroecology and biogeography: state-of-art and prospects. Ecography, 40(2):267-280.

Caddy-Retalic, S., Hoffmann, B. D., Guerin, G. R., Andersen, A. N., Wardle, G. M., Mclnerney, F. A., and Lowe, A. J. (2018). Plant and ant assemblages predicted to decouple under climate change. Diversity and Distributions, 00(0):1-17.

Cahill, A. E., Aiello-Lammens Matthew E., Fisher-Reid M. Caitlin, Hua Xia, Karanewsky Caitlin J., Yeong Ryu Hae, Sbeglia Gena C., Spagnolo Fabrizio, Waldron John B., Warsi Omar, and Wiens John J. (2013). How does climate change cause extinction? Proceedings of the Royal Society B: Biological Sciences, 280(1750):20121890.

Caño, L., Escarré, J., and Sans, F. X. (2007). Factors affecting the invasion success of Senecio inaequidens and S. pterophorus in Mediterranean plant communities. Journal of Vegetation Science, 18(2):281-288.

Cappuccino, N. and Carpenter, D. (2005). Invasive exotic plants suffer less herbivory than non-invasive exotic plants. Biology Letters, 1(4):435-438.

Catford, J. A., Smith, A. L., Wragg, P. D., Clark, A. T., Kosmala, M., Cavender-Bares, J., Reich, P. B., and Tilman, D. (2019). Traits linked with species invasiveness and community invasibility vary with time, stage and indicator of invasion in a long-term grassland experiment. Ecology Letters, 22(4):593-604. 
Cavin, L., Mountford, E. P., Peterken, G. F., and Jump, A. S. (2013). Extreme drought alters competitive dominance within and between tree species in a mixed forest stand. Functional Ecology, 27(6):1424-1435.

Chapin, F. S., Randerson, J. T., McGuire, A. D., Foley, J. A., and Field, C. B. (2008). Changing feedbacks in the climate-biosphere system. Frontiers in Ecology and the Environment, 6(6):313-320.

Chave, J. (2013). The problem of pattern and scale in ecology: what have we learned in 20 years? Ecology Letters, 16(s1):4-16.

Chen, I.-C., Hill, J. K., Ohlemüller, R., Roy, D. B., and Thomas, C. D. (2011). Rapid Range Shifts of Species Associated with High Levels of Climate Warming. Science, 333(6045):1024-1026.

Cheptou, P.-O., Carrue, O., Rouifed, S., and Cantarel, A. (2008). Rapid evolution of seed dispersal in an urban environment in the weed Crepis sancta. Proceedings of the National Academy of Sciences, 105(10):3796-3799.

Cheptou, P.-O., Hargreaves, A. L., Bonte, D., and Jacquemyn, H. (2017). Adaptation to fragmentation: evolutionary dynamics driven by human influences. Philosophical Transactions of the Royal Society B: Biological Sciences, 372(1712):20160037.

Cheruvelil, K. S. and Soranno, P. A. (2018). Data-Intensive Ecological Research Is Catalyzed by Open Science and Team Science. BioScience, 68(10):813-822.

Cheruvelil, K. S., Yuan, S., Webster, K. E., Tan, P.-N., Lapierre, J.-F., Collins, S. M., Fergus, C. E., Scott, C. E., Henry, E. N., Soranno, P. A., Filstrup, C. T., and Wagner, T. (2017). Creating multithemed ecological regions for macroscale ecology: Testing a flexible, repeatable, and accessible clustering method. Ecology and Evolution, 7(9):30463058.

Chu, C.-J., Maestre, F. T., Xiao, S., Weiner, J., Wang, Y.-S., Duan, Z.-H., and Wang, G. (2008). Balance between facilitation and resource competition determines biomass-density relationships in plant populations. Ecology Letters, 11(11):1189-1197.

Chun, Y. J., Kleunen, M. V., and Dawson, W. (2010). The role of enemy release, tolerance and resistance in plant invasions: linking damage to performance. Ecology Letters, 13(8):937-946.

$\mathrm{Ci}, \mathrm{L}$. and Yang, X. (2004). Progress in feedback mechanism between desertification and climate change. Acta Ecologica Sinica, 24(4):755-760.

Cobben, M., Verboom, J., Opdam, P., Hoekstra, R., Jochem, R., and Smulders, M. (2012). Wrong place, wrong time: climate change-induced range shift across fragmented habitat causes maladaptation and declined population size in a modelled bird species. Global Change Biology.

Colbach, N. and Sache, I. (2001). Blackgrass (Alopecurus myosuroides Huds.) seed dispersal from a single plant and its consequences on weed infestation. Ecological Modelling, 139(2):201-219.

Colwell, R. K., Brehm, G., Cardelús, C. L., Gilman, A. C., and Longino, J. T. (2008). Global Warming, Elevational Range Shifts, and Lowland Biotic Attrition in the Wet Tropics. Science, 322(5899):258-261. 


\section{LITERATURE}

Compant, S., Van Der Heijden, M. G. A., and Sessitsch, A. (2010). Climate change effects on beneficial plant-microorganism interactions. FEMS Microbiology Ecology, 73(2):197-214.

Connolly, J. and Wayne, P. (1996). Asymmetric competition between plant species. Oecologia, 108(2):311-320. WOS:A1996VN74300015.

Copeland, J. H., Pielke, R. A., and Kittel, T. G. F. (1996). Potential climatic impacts of vegetation change: A regional modeling study. Journal of Geophysical Research: Atmospheres, 101(D3):7409-7418.

Corbin, J. D. and D'Antonio, C. M. (2004). Competition between native perennial and exotic annual grasses: implications for an historical invasion. Ecology, 85(5):1273-1283.

Corlett, R. T. and Westcott, D. A. (2013). Will plant movements keep up with climate change? Trends in Ecology \& Evolution, 28(8):482-488.

Cressie, N., Calder, C. A., Clark, J. S., Hoef, J. M. V., and Wikle, C. K. (2009). Accounting for uncertainty in ecological analysis: the strengths and limitations of hierarchical statistical modeling. Ecological Applications, 19(3):553-570.

Cunze, S., Heydel, F., and Tackenberg, O. (2013). Are Plant Species Able to Keep Pace with the Rapidly Changing Climate? PLoS ONE, 8(7):e67909.

DAISIE (2012). European Invasive Alien Species Gateway.

Dakos, V., Matthews, B., Hendry, A. P., Levine, J., Loeuille, N., Norberg, J., Nosil, P., Scheffer, M., and Meester, L. D. (2019). Ecosystem tipping points in an evolving world. Nature Ecology \& Evolution, 3(3):355.

Damgaard, C. and Weiner, J. (2008). Modeling the growth of individuals in crowded plant populations. Journal of Plant Ecology, 1(2):111-116. WOS:000265790100005.

Darwin, C. (1859). On the Origin of Species. Routledge.

Davin, E. L. and de Noblet-Ducoudré, N. (2010). Climatic Impact of Global-Scale Deforestation: Radiative versus Nonradiative Processes. Journal of Climate, 23(1):97-112.

Dawson, T. P., Jackson, S. T., House, J. I., Prentice, I. C., and Mace, G. M. (2011). Beyond Predictions: Biodiversity Conservation in a Changing Climate. Science, 332(6025):5358.

DeAngelis, D. L. and Grimm, V. (2014). Individual-based models in ecology after four decades. F1000Prime Reports, 6.

Deser, C., Walsh, J. E., and Timlin, M. S. (2000). Arctic Sea Ice Variability in the Context of Recent Atmospheric Circulation Trends. Journal of Climate, 13(3):617-633.

Diffenbaugh, N. S. and Field, C. B. (2013). Changes in Ecologically Critical Terrestrial Climate Conditions. Science, 341(6145):486-492.

Dislich, C., Johst, K., and Huth, A. (2010). What enables coexistence in plant communities? Weak versus strong species traits and the role of local processes. Ecological Modelling, 221(19):2227-2236.

Domingos, P. (1999). The Role of Occam's Razor in Knowledge Discovery. Data Mining and Knowledge Discovery, 3(4):409-425. 
Dormann, C. F., Schymanski, S. J., Cabral, J., Chuine, I., Graham, C., Hartig, F., Kearney, M., Morin, X., Römermann, C., Schröder, B., and Singer, A. (2012). Correlation and process in species distribution models: bridging a dichotomy. Journal of Biogeography, 39(12):2119-2131.

Dostál, P. (2011). Plant Competitive Interactions and Invasiveness: Searching for the Effects of Phylogenetic Relatedness and Origin on Competition Intensity. The American Naturalist, 177(5):655-667.

Duputié, A., Zimmermann, N. E., and Chuine, I. (2014). Where are the wild things? Why we need better data on species distribution. Global Ecology and Biogeography, 23(4):457-467.

Eatough Jones, M., Paine, T. D., Fenn, M. E., and Poth, M. A. (2004). Influence of ozone and nitrogen deposition on bark beetle activity under drought conditions. Forest Ecology and Management, 200(1):67-76.

Edwards, G. and Crawley, M. (1999). Herbivores, seed banks and seedling recruitment in mesic grassland. Journal of Ecology, 87(3):423-435.

Ehlers, B. K. (2011). Soil microorganisms alleviate the allelochemical effects of a thyme monoterpene on the performance of an associated grass species. PloS one, 6(11):e26321.

Ehrlén, J. and Morris, W. F. (2015). Predicting changes in the distribution and abundance of species under environmental change. Ecology Letters, 18(3):303-314.

Elith, J., Kearney, M., and Phillips, S. (2010). The art of modelling range-shifting species. Methods in ecology and evolution, 1(4):330-342.

Engelkes, T., Morrien, E., Verhoeven, K. J. F., Bezemer, T. M., Biere, A., Harvey, J. A., Mclntyre, L. M., Tamis, W. L. M., and van der Putten, W. H. (2008). Successful range-expanding plants experience less above-ground and below-ground enemy impact. Nature, 456(7224):946-948.

Engler, R. and Guisan, A. (2009). MigClim: predicting plant distribution and dispersal in a changing climate. Diversity and Distributions, 15(4):590-601.

Ernakovich, J. G., Hopping, K. A., Berdanier, A. B., Simpson, R. T., Kachergis, E. J., Steltzer, H., and Wallenstein, M. D. (2014). Predicted responses of arctic and alpine ecosystems to altered seasonality under climate change. Global Change Biology, 20(10):3256-3269.

Erneberg, M. (1999). Effects of herbivory and competition on an introduced plant in decline. Oecologia, 118(2):203-209. WOS:000078978400011.

Estrada, A., Morales-Castilla, I., Caplat, P., and Early, R. (2016). Usefulness of Species Traits in Predicting Range Shifts. Trends in Ecology \& Evolution, 31(3):190-203.

Estrada, A., Morales-Castilla, I., Meireles, C., Caplat, P., and Early, R. (2018). Equipped to cope with climate change: traits associated with range filling across European taxa. Ecography, 41(5):770-781.

Evans, M. R., Grimm, V., Johst, K., Knuuttila, T., de Langhe, R., Lessells, C. M., Merz, M., O'Malley, M. A., Orzack, S. H., Weisberg, M., Wilkinson, D. J., Wolkenhauer, O., and Benton, T. G. (2013). Do simple models lead to generality in ecology? Trends in Ecology \& Evolution, 28(10):578-583. 


\section{LITERATURE}

Fagan, W. F., Lewis, M., Neubert, M. G., Aumann, C., Apple, J. L., and Bishop, J. G. (2005). When Can Herbivores Slow or Reverse the Spread of an Invading Plant? A Test Case from Mount St. Helens. The American Naturalist, 166(6):669-685.

Farley, S. S., Dawson, A., Goring, S. J., and Williams, J. W. (2018). Situating Ecology as a Big-Data Science: Current Advances, Challenges, and Solutions. BioScience, 68(8):563576.

Fei, S., Desprez, J. M., Potter, K. M., Jo, I., Knott, J. A., and Oswalt, C. M. (2017). Divergence of species responses to climate change. Science Advances, 3(5):e1603055.

Fei, S., Guo, Q., and Potter, K. (2016). Macrosystems ecology: novel methods and new understanding of multi-scale patterns and processes. Landscape Ecology, 31(1):1-6.

Fernández, M., Hamilton, H., Alvarez, O., and Guo, Q. (2012). Does adding multi-scale climatic variability improve our capacity to explain niche transferability in invasive species? Ecological Modelling, 246:60-67.

Foley, J. A., Levis, S., Costa, M. H., Cramer, W., and Pollard, D. (2000). Incorporating Dynamic Vegetation Cover Within Global Climate Models. Ecological Applications, 10(6):1620-1632.

Fornoni, J. (2011). Ecological and evolutionary implications of plant tolerance to herbivory. Functional Ecology, 25(2):399-407. WOS:000288501100010.

Fox, L. R., Ribeiro, S. P., Brown, V. K., Masters, G. J., and Clarke, I. P. (1999). Direct and indirect effects of climate change on St John's wort, Hypericum perforatum L.(Hypericaceae). Oecologia, 120(1):113-122.

Francis, J. A., Skific, N., and Vavrus, S. J. (2018). North American Weather Regimes Are Becoming More Persistent: Is Arctic Amplification a Factor? Geophysical Research Letters, 45(20):11,414-11,422.

Franklin, J., Davis, F. W., Ikegami, M., Syphard, A. D., Flint, L. E., Flint, A. L., and Hannah, L. (2013). Modeling plant species distributions under future climates: how fine scale do climate projections need to be? Global Change Biology, 19(2):473-483.

Franks, S. J., Sim, S., and Weis, A. E. (2007). Rapid evolution of flowering time by an annual plant in response to a climate fluctuation. Proceedings of the National Academy of Sciences, 104(4):1278-1282.

Franks, S. J., Weber, J. J., and Aitken, S. N. (2014). Evolutionary and plastic responses to climate change in terrestrial plant populations. Evolutionary Applications, 7(1):123-139.

Fraterrigo, J. M., Wagner, S., and Warren, R. J. (2014). Local-scale biotic interactions embedded in macroscale climate drivers suggest Eltonian noise hypothesis distribution patterns for an invasive grass. Ecology Letters, 17(11):1447-1454.

Freeman, B. G., Lee-Yaw, J. A., Sunday, J. M., and Hargreaves, A. L. (2018). Expanding, shifting and shrinking: The impact of global warming on species' elevational distributions. Global Ecology and Biogeography, 27(11):1268-1276.

Gallagher, R. V., Makinson, R. O., Hogbin, P. M., and Hancock, N. (2015). Assisted colonization as a climate change adaptation tool. Austral Ecology, 40(1):12-20.

García-Palacios, P., Gross, N., Gaitán, J., and Maestre, F. T. (2018). Climate mediates the biodiversity-ecosystem stability relationship globally. Proceedings of the National Academy of Sciences, 115(33):8400-8405. 
Gehrig-Fasel, J., Guisan, A., and Zimmermann, N. E. (2007). Tree line shifts in the Swiss Alps: Climate change or land abandonment? Journal of Vegetation Science, 18(4):571582.

Geist, H. J. and Lambin, E. F. (2004). Dynamic Causal Patterns of Desertification. BioScience, 54(9):817-829.

Gerhardt, F. and Collinge, S. K. (2007). Abiotic constraints eclipse biotic resistance in determining invasibility along experimental vernal pool gradients. Ecological Applications, 17(3):922-933.

Gettelman, A. and Rood, R. B. (2016). Components of the Climate System. In Gettelman, A. and Rood, R. B., editors, Demystifying Climate Models: A Users Guide to Earth System Models, Earth Systems Data and Models, pages 13-22. Springer Berlin Heidelberg, Berlin, Heidelberg.

Gilman, S. E., Urban, M. C., Tewksbury, J., Gilchrist, G. W., and Holt, R. D. (2010). A framework for community interactions under climate change. Trends in Ecology \& Evolution, 25(6):325-331.

Goldberg, D. and Landa, K. (1991). Competitive Effect and Response - Hierarchies and Correlated Traits in the Early Stages of Competition. Journal of Ecology, 79(4):10131030. WOS:A1991HQ47400009.

Goldberg, D. E. (1996). Competitive ability: Definitions, contingency and correlated traits. Philosophical Transactions of the Royal Society of London Series B-Biological Sciences, 351(1345):1377-1385. WOS:A1996VL43700018.

Gonzales, E. K. and Arcese, P. (2008). Herbivory more limiting than competition on early and established native plants in an invaded meadow. Ecology, 89(12):3282-3289.

Goring, S. J., Weathers, K. C., Dodds, W. K., Soranno, P. A., Sweet, L. C., Cheruvelil, K. S., Kominoski, J. S., Rüegg, J., Thorn, A. M., and Utz, R. M. (2014). Improving the culture of interdisciplinary collaboration in ecology by expanding measures of success. Frontiers in Ecology and the Environment, 12(1):39-47.

Grace, J., Berninger, F., and Nagy, L. (2002). Impacts of Climate Change on the Tree Line. Annals of Botany, 90(4):537-544.

Gray, S. B. and Brady, S. M. (2016). Plant developmental responses to climate change. Developmental Biology, 419(1):64-77.

Green, J. K., Konings, A. G., Alemohammad, S. H., Berry, J., Entekhabi, D., Kolassa, J., Lee, J.-E., and Gentine, P. (2017). Regionally strong feedbacks between the atmosphere and terrestrial biosphere. Nature Geoscience, 10(6):410-414.

Grimm, V., Berger, U., Bastiansen, F., Eliassen, S., Ginot, V., Giske, J., Goss-Custard, J., Grand, T., Heinz, S. K., Huse, G., Huth, A., Jepsen, J. U., Jørgensen, C., Mooij, W. M., Müller, B., Pe'er, G., Piou, C., Railsback, S. F., Robbins, A. M., Robbins, M. M., Rossmanith, E., Rüger, N., Strand, E., Souissi, S., Stillman, R. A., Vabø, R., Visser, U., and DeAngelis, D. L. (2006). A standard protocol for describing individual-based and agent-based models. Ecological Modelling, 198(1-2):115-126.

Grimm, V., Berger, U., DeAngelis, D. L., Polhill, J. G., Giske, J., and Railsback, S. F. (2010). The ODD protocol: A review and first update. Ecological Modelling, 221(23):2760-2768. 


\section{LITERATURE}

Grimm, V. and Railsback, S. F. (2005). Individual-based Modeling And Ecology. Princeton University Press.

Grimm, V., Revilla, E., Berger, U., Jeltsch, F., Mooij, W. M., Railsback, S. F., Thulke, H.H., Weiner, J., Wiegand, T., and DeAngelis, D. L. (2005). Pattern-Oriented Modeling of Agent-Based Complex Systems: Lessons from Ecology. Science, 310(5750):987-991.

Groffman, P. M., Baron, J. S., Blett, T., Gold, A. J., Goodman, I., Gunderson, L. H., Levinson, B. M., Palmer, M. A., Paerl, H. W., Peterson, G. D., Poff, N. L., Rejeski, D. W., Reynolds, J. F., Turner, M. G., Weathers, K. C., and Wiens, J. (2006). Ecological Thresholds: The Key to Successful Environmental Management or an Important Concept with No Practical Application? Ecosystems, 9(1):1-13.

Gross, N., Börger, L., Duncan, R. P., and Hulme, P. E. (2013). Functional differences between alien and native species: do biotic interactions determine the functional structure of highly invaded grasslands? Functional Ecology, 27(5):1262-1272.

Gross, N., Kunstler, G., Liancourt, P., Bello, F. D., Suding, K. N., and Lavorel, S. (2009). Linking individual response to biotic interactions with community structure: a trait-based framework. Functional Ecology, 23(6):1167-1178.

Guerin, G. R., Wen, H., and Lowe, A. J. (2012). Leaf morphology shift linked to climate change. Biology Letters, 8(5):882-886.

Guisan, A. and Thuiller, W. (2005). Predicting species distribution: offering more than simple habitat models. Ecology letters, 8(9):993-1009.

Hampe, A. (2011). Plants on the move: The role of seed dispersal and initial population establishment for climate-driven range expansions. Acta Oecologica-International Journal of Ecology, 37(6):666-673. WOS:000297955800018.

Hampe, A. and Petit, R. J. (2005). Conserving biodiversity under climate change: the rear edge matters. Ecology Letters, 8(5):461-467.

Hannah, L., Flint, L., Syphard, A. D., Moritz, M. A., Buckley, L. B., and McCullough, I. M. (2014). Fine-grain modeling of species' response to climate change: holdouts, stepping-stones, and microrefugia. Trends in Ecology \& Evolution, 29(7):390-397.

Hargreaves, A. L., Bailey, S. F., and Laird, R. A. (2015). Fitness declines towards range limits and local adaptation to climate affect dispersal evolution during climate-induced range shifts. Journal of Evolutionary Biology, 28(8):1489-1501.

Harvey, J. and Fortuna, T. (2012). Chemical and structural effects of invasive plants on herbivore-parasitoid/predator interactions in native communities. Entomologia Experimentalis et Applicata, (144).

Harvey, J. A., Bukovinszky, T., and van der Putten, W. H. (2010). Interactions between invasive plants and insect herbivores: A plea for a multitrophic perspective. Biological Conservation, 143(10):2251-2259. WOS:000282076800002.

Hattermann, F. F., Weiland, M., Huang, S., Krysanova, V., and Kundzewicz, Z. W. (2011). Model-Supported Impact Assessment for the Water Sector in Central Germany Under Climate Change-A Case Study. Water Resources Management, 25(13):3113.

Hauggaard-Nielsen, H. and Jensen, E. (2005). Facilitative root interactions in intercrops. In Lambers, H. and Colmer, T. D., editors, Root Physiology: from Gene to Function, Plant Ecophysiology, pages 237-250. Springer Netherlands, Dordrecht. 
Hautier, Y., Tilman, D., Isbell, F., Seabloom, E. W., Borer, E. T., and Reich, P. B. (2015). Anthropogenic environmental changes affect ecosystem stability via biodiversity. Science, 348(6232):336-340.

Heard, M. J. and Sax, D. F. (2013). Coexistence between native and exotic species is facilitated by asymmetries in competitive ability and susceptibility to herbivores. Ecology Letters, 16(2):206-213.

Heffernan, J. B., Soranno, P. A., Angilletta, M. J., Buckley, L. B., Gruner, D. S., Keitt, T. H., Kellner, J. R., Kominoski, J. S., Rocha, A. V., Xiao, J., Harms, T. K., Goring, S. J., Koenig, L. E., McDowell, W. H., Powell, H., Richardson, A. D., Stow, C. A., Vargas, R., and Weathers, K. C. (2014). Macrosystems ecology: understanding ecological patterns and processes at continental scales. Frontiers in Ecology and the Environment, 12(1):514.

Hefley, T. J., Hooten, M. B., Russell, R. E., Walsh, D. P., and Powell, J. A. (2017). When mechanism matters: Bayesian forecasting using models of ecological diffusion. Ecology Letters, 20(5):640-650.

Heikkinen, R. K., Luoto, M., Araújo, M. B., Virkkala, R., Thuiller, W., and Sykes, M. T. (2006). Methods and uncertainties in bioclimatic envelope modelling under climate change. Progress in Physical Geography: Earth and Environment, 30(6):751-777.

Hejda, M., Chytrý, M., Pergl, J., and Pyšek, P. (2015). Native-range habitats of invasive plants: are they similar to invaded-range habitats and do they differ according to the geographical direction of invasion? Diversity and Distributions, 21(3):312-321.

Herrero, A., Almaraz, P., Zamora, R., Castro, J., and Hódar, J. A. (2016). From the individual to the landscape and back: time-varying effects of climate and herbivory on tree sapling growth at distribution limits. Journal of Ecology, 104(2):430-442.

Hierro, J. L. and Callaway, R. M. (2003). Allelopathy and exotic plant invasion. Plant and Soil, 256(1):29-39.

Hierro, J. L., Eren, Ö., Villarreal, D., and Chiuffo, M. C. (2013). Non-native conditions favor non-native populations of invasive plant: demographic consequences of seed size variation? Oikos, 122(4):583-590.

Hoffmann, A. A. and Sgrò, C. M. (2011). Climate change and evolutionary adaptation. Nature, 470(7335):479-485.

Holle, B. and Simberloff, D. (2005). Ecological resistance to biological invasion overwhelmed by propagule pressure. Ecology, 86(12):3212-3218.

Holyoak, M. and Heath, S. K. (2016). The integration of climate change, spatial dynamics, and habitat fragmentation: A conceptual overview. Integrative Zoology, 11(1):40-59.

Honnay, O., Verheyen, K., Butaye, J., Jacquemyn, H., Bossuyt, B., and Hermy, M. (2002). Possible effects of habitat fragmentation and climate change on the range of forest plant species. Ecology Letters, 5(4):525-530.

Hooten, M. B. and Wikle, C. K. (2008). A hierarchical Bayesian non-linear spatio-temporal model for the spread of invasive species with application to the Eurasian Collared-Dove. Environmental and Ecological Statistics, 15(1):59-70. 


\section{LITERATURE}

Hovick, S. M., Peterson, C. J., and Carson, W. P. (2012). Predicting invasiveness and range size in wetland plants using biological traits: a multivariate experimental approach. Journal of Ecology, 100(6):1373-1382. WOS:000310336500009.

Howard, T. G. and Goldberg, D. E. (2001). Competitive response hierarchies for germination, growth, and survival and their influence on abundance. Ecology, 82(4):979-990. WOS:000168135100007.

Huang, W., Carrillo, J., Ding, J., and Siemann, E. (2012). Interactive effects of herbivory and competition intensity determine invasive plant performance. Oecologia, 170(2):373382. WOS:000308662100009.

Hulme, P. (2007). Biological invasions in Europe: drivers, pressures, states, impacts and responses. Biodiversity under threat issues in environmental science and technology, 25:56-80.

Hunt, R. (1982). Plant growth curves. The functional approach to plant growth analysis. Plant growth curves. The functional approach to plant growth analysis.

Huntley, B., Berry, P., Cramer, W., and McDonald, A. (1995). Special Paper: Modelling Present and Potential Future Ranges of Some European Higher Plants Using Climate Response Surfaces. Journal of Biogeography, pages 967-1001.

IPCC (2014). Climate Change 2014: Synthesis Report. Contribution of Working Groups I, II and III to the Fifth Assessment Report of the Intergovernmental Panel on Climate Change [Core Writing Team, R.K. Pachauri and L.A. Meyer (eds.)]. Geneva, Switzerland.

Isbell, F., Craven, D., Connolly, J., Loreau, M., Schmid, B., Beierkuhnlein, C., Bezemer, T. M., Bonin, C., Bruelheide, H., de Luca, E., Ebeling, A., Griffin, J. N., Guo, Q., Hautier, Y., Hector, A., Jentsch, A., Kreyling, J., Lanta, V., Manning, P., Meyer, S. T., Mori, A. S., Naeem, S., Niklaus, P. A., Polley, H. W., Reich, P. B., Roscher, C., Seabloom, E. W., Smith, M. D., Thakur, M. P., Tilman, D., Tracy, B. F., van der Putten, W. H., van Ruijven, J., Weigelt, A., Weisser, W. W., Wilsey, B., and Eisenhauer, N. (2015). Biodiversity increases the resistance of ecosystem productivity to climate extremes. Nature, 526(7574):574-577.

Jackson, S. T. and Sax, D. F. (2010). Balancing biodiversity in a changing environment: extinction debt, immigration credit and species turnover. Trends in Ecology \& Evolution, 25(3):153-160.

Jakobsson, A. and Eriksson, O. (2000). A comparative study of seed number, seed size, seedling size and recruitment in grassland plants. Oikos, 88(3):494-502.

Janeček, Š., Patáčová, E., and Klimešová, J. (2014). Effects of Fertilization and Competition on Plant Biomass Allocation and Internal Resources: Does Plantago lanceolata Follow the Rules of Economic Theory? Folia Geobotanica, 49(1):49-64.

Jeschke, J., Gómez Aparicio, L., Haider, S., Heger, T., Lortie, C., Pyšek, P., and Strayer, D. (2012). Support for major hypotheses in invasion biology is uneven and declining. NeoBiota, 14:1-20.

Johnson, N. C., Wilson, G. W. T., Bowker, M. A., Wilson, J. A., and Miller, R. M. (2010). Resource limitation is a driver of local adaptation in mycorrhizal symbioses. Proceedings of the National Academy of Sciences, 107(5):2093-2098. 
Joshi, J. and Vrieling, K. (2005). The enemy release and EICA hypothesis revisited: incorporating the fundamental difference between specialist and generalist herbivores. Ecology Letters, 8(7):704-714.

Jump, A. and Penuelas, J. (2005). Running to stand still: adaptation and the response of plants to rapid climate change. Ecology Letters, 8(9):1010-1020.

Kanakidou, M., Myriokefalitakis, S., Daskalakis, N., Fanourgakis, G., Nenes, A., Baker, A. R., Tsigaridis, K., and Mihalopoulos, N. (2016). Past, Present, and Future Atmospheric Nitrogen Deposition. Journal of the Atmospheric Sciences, 73(5):2039-2047.

Karrasch, H. (1973). Microclimatic Studies in the ALPS. Arctic and Alpine Research, 5(sup3):A55-A63.

Keane, R. and Crawley, M. (2002). Exotic plant invasions and the enemy release hypothesis. Trends in Ecology \& Evolution, 17(4):164-170.

Kearney, M., Simpson, S. J., Raubenheimer, D., and Helmuth, B. (2010). Modelling the ecological niche from functional traits. Philosophical Transactions of the Royal Society B: Biological Sciences, 365(1557):3469-3483.

Kelly, J. F. and Horton, K. G. (2016). Toward a predictive macrosystems framework for migration ecology. Global Ecology and Biogeography, 25(10):1159-1165.

Kempel, A., Chrobock, T., Fischer, M., Rohr, R. P., and Kleunen, M. v. (2013). Determinants of plant establishment success in a multispecies introduction experiment with native and alien species. Proceedings of the National Academy of Sciences, 110(31):1272712732.

Kessler, A. and Baldwin, I. T. (2001). Defensive Function of Herbivore-Induced Plant Volatile Emissions in Nature. Science, 291(5511):2141-2144.

Kim, T. N., Underwood, N., and Inouye, B. D. (2013). Insect herbivores change the outcome of plant competition through both inter- and intraspecific processes. Ecology, 94(8):1753-1763.

Kinlan, B. and Hastings, A. (2005). What exotic species tell us about rates of population spread and geographic range expansion. Species invasions: insights to ecology, evolution and biogeography, pages 381-419.

Klanderud, K. (2005). Climate change effects on species interactions in an alpine plant community. Journal of Ecology, 93(1):127-137.

Kolar, C. and Lodge, D. (2001). Progress in invasion biology: predicting invaders. Trends in Ecology \& Evolution, 16(4):199-204.

Köppen, W. (1884). Die Wärmezonen der Erde, nach der Dauer der heissen, gemässigten und kalten Zeit und nach der Wirkung der Wärme auf die organische Welt betrachtet. Meteorologische Zeitschrift, 1(21):5-226.

Kress, M. M. (2019). Big Data for Ecological Models. In Fath, B., editor, Encyclopedia of Ecology (Second Edition), pages 11-20. Elsevier, Oxford.

Kreyling, J. (2010). Winter climate change: a critical factor for temperate vegetation performance. Ecology, 91(7):1939-1948.

Kuijper, D., Nijhoff, D., and Bakker, J. (2004). Herbivory and competition slow down invasion of a tall grass along a productivity gradient. Oecologia, 141(3). 


\section{LITERATURE}

Kunstler, G., Falster, D., Coomes, D. A., Hui, F., Kooyman, R. M., Laughlin, D. C., Poorter, L., Vanderwel, M., Vieilledent, G., Wright, S. J., Aiba, M., Baraloto, C., Caspersen, J., Cornelissen, J. H. C., Gourlet-Fleury, S., Hanewinkel, M., Herault, B., Kattge, J., Kurokawa, H., Onoda, Y., Peñuelas, J., Poorter, H., Uriarte, M., Richardson, S., RuizBenito, P., Sun, I.-F., Ståhl, G., Swenson, N. G., Thompson, J., Westerlund, B., Wirth, C., Zavala, M. A., Zeng, H., Zimmerman, J. K., Zimmermann, N. E., and Westoby, M. (2016). Plant functional traits have globally consistent effects on competition. Nature, 529(7585):204-207.

Küster, E. C., Kühn, I., Bruelheide, H., and Klotz, S. (2008). Trait interactions help explain plant invasion success in the German flora. Journal of Ecology, 96(5):860-868.

Lakeman-Fraser, P. and Ewers, R. M. (2013). Enemy release promotes range expansion in a host plant. Oecologia, 172(4):1203-1212. WOS:000322180000027.

Lambdon, P. W., Pyšek, P., Basnou, C., Hejda, M., Arianoutsou, M., Essl, F., Jarošík, V., Pergl, J., Winter, M., Anastasiu, P., Andriopoulos, P., Bazos, I., Brundu, G., CelestiGrapow, L., Chassot, P., Delipetrou, P., Josefsson, M., Kark, S., Klotz, S., Kokkoris, Y., Kühn, I., Marchante, H., Perglová, I., Pino, J., Vila, M., and Zikos, A. e. a. (2008). Alien flora of Europe: species diversity, temporal trends, geographical patterns and research needs. Preslia, 80(2):101-149.

Larson, D., Anderson, P., and Newton, W. (2001). Alien plant invasion in mixed-grass prairie: effects of vegetation type and anthropogenic disturbance. Ecological Applications, 11(1):128-141.

le Roux, P. C. and McGeoch, M. A. (2008). Rapid range expansion and community reorganization in response to warming. Global Change Biology, 14(12):2950-2962.

le Roux, P. C. I., Virtanen, R., Heikkinen, R. K., and Luoto, M. (2012). Biotic interactions affect the elevational ranges of high-latitude plant species. Ecography, 35(11):10481056.

Ledergerber, S., Thommen, G. H., and Baur, B. (1997). Grazing damage to plants and gastropod and grasshopper densities in a $\mathrm{CO} 2$-enrichment experiment on calcareous grassland. Acta Oecologica, 18(3):255-261.

Leishman, M. R. (2001). Does the seed size/number trade-off model determine plant community structure? an assessment of the model mechanisms and their generality. Oikos, 93(2):294-302.

Leishman, M. R., Wright, I. J., Moles, A. T., Westoby, M., and others (2000). The evolutionary ecology of seed size. Seeds: the ecology of regeneration in plant communities, 2:31-57.

Lenoir, J., Gégout, J.-C., Guisan, A., Vittoz, P., Wohlgemuth, T., Zimmermann, N. E., Dullinger, S., Pauli, H., Willner, W., and Svenning, J.-C. (2010). Going against the flow: potential mechanisms for unexpected downslope range shifts in a warming climate. Ecography, 33(2):295-303.

Lenoir, J., Gégout, J. C., Marquet, P. A., Ruffray, P. d., and Brisse, H. (2008). A Significant Upward Shift in Plant Species Optimum Elevation During the 20th Century. Science, 320(5884):1768-1771.

Lenoir, J. and Svenning, J.-C. (2015). Climate-related range shifts-a global multidimensional synthesis and new research directions. Ecography, 38(1):15-28. 
Lenton, T. M., Held, H., Kriegler, E., Hall, J. W., Lucht, W., Rahmstorf, S., and Schellnhuber, H. J. (2008). Tipping elements in the Earth's climate system. Proceedings of the National Academy of Sciences, 105(6):1786-1793.

Levin, S. A. (1992). The Problem of Pattern and Scale in Ecology: The Robert H. MacArthur Award Lecture. Ecology, 73(6):1943-1967.

Levine, J. M., Adler, P. B., and Yelenik, S. G. (2004). A meta-analysis of biotic resistance to exotic plant invasions. Ecology Letters, 7(10):975-989.

Levy, O., Ball, B. A., Bond-Lamberty, B., Cheruvelil, K. S., Finley, A. O., Lottig, N. R., Punyasena, S. W., Xiao, J., Zhou, J., Buckley, L. B., Filstrup, C. T., Keitt, T. H., Kellner, J. R., Knapp, A. K., Richardson, A. D., Tcheng, D., Toomey, M., Vargas, R., Voordeckers, J. W., Wagner, T., and Williams, J. W. (2014). Approaches to advance scientific understanding of macrosystems ecology. Frontiers in Ecology and the Environment, 12(1):15-23.

Lin, B. B., Chappell, M. J., Vandermeer, J., Smith, G., Quintero, E., Bezner-Kerr, R., Griffith, D. M., Ketcham, S., Latta, S. C., McMichael, P., McGuire, K. L., Nigh, R., Rocheleau, D., Soluri, J., and Perfecto, I. (2011). Effects of industrial agriculture on climate change and the mitigation potential of small-scale agro-ecological farms. $C A B$ Reviews: Perspectives in Agriculture, Veterinary Science, Nutrition and Natural Resources, 6(020):1-18.

Lin, Y., Berger, U., Grimm, V., and Ji, Q.-R. (2012). Differences between symmetric and asymmetric facilitation matter: exploring the interplay between modes of positive and negative plant interactions. Journal of Ecology, 100(6):1482-1491.

Lischke, H., Löffler, T. J., Thornton, P. E., and Zimmermann, N. E. (2007). Model Upscaling in Landscape Research. In Kienast, F., Wildi, O., and Ghosh, S., editors, A Changing World, number 8 in Landscape Series, pages 249-272. Springer Netherlands.

Loarie, S. R., Duffy, P. B., Hamilton, H., Asner, G. P., Field, C. B., and Ackerly, D. D. (2009). The velocity of climate change. Nature, 462(7276):1052-1055.

Lockwood, J., Cassey, P., and Blackburn, T. (2005). The role of propagule pressure in explaining species invasions. Trends in Ecology \& Evolution, 20(5):223-228.

Loon, J. J. A. v., Boer, J. G. d., and Dicke, M. (2000). Parasitoid-plant mutualism: parasitoid attack of herbivore increases plant reproduction. Entomologia Experimentalis et Applicata, 97(2):219-227.

Lu, X. and Ding, J. (2012). History of exposure to herbivores increases the compensatory ability of an invasive plant. Biological Invasions, (14):649-658.

Ma, Z., Liu, H., Mi, Z., Zhang, Z., Wang, Y., Xu, W., Jiang, L., and He, J.-S. (2017). Climate warming reduces the temporal stability of plant community biomass production. Nature Communications, 8.

Macel, M., Bruinsma, M., Dijkstra, S., Ooijendijk, T., Niemeyer, H., and Klinkhamer, P. (2005). Differences in effects of pyrrolizidine alkaloids on five generalist insect herbivore species. Journal of Chemical Ecology, 31(7):1493-1508.

Mackey, B. G. and Lindenmayer, D. B. (2001). Towards a hierarchical framework for modelling the spatial distribution of animals. Journal of Biogeography, 28(9):11471166. 


\section{LITERATURE}

MacLean, S. A. and Beissinger, S. R. (2017). Species' traits as predictors of range shifts under contemporary climate change: A review and meta-analysis. Global Change Biology, 23(10):4094-4105.

Májeková, M., de Bello, F., Doležal, J., and Lepš, J. (2014). Plant functional traits as determinants of population stability. Ecology.

Malhi, Y., Aragão, L. E. O. C., Galbraith, D., Huntingford, C., Fisher, R., Zelazowski, P., Sitch, S., McSweeney, C., and Meir, P. (2009). Exploring the likelihood and mechanism of a climate-change-induced dieback of the Amazon rainforest. Proceedings of the National Academy of Sciences, 106(49):20610-20615.

Marini, L., Bruun, H., Heikkinen, R., Helm, A., Honnay, O., Krauss, J., Kühn, I., Lindborg, R., Pärtel, M., and Bommarco, R. (2012). Traits related to species persistence and dispersal explain changes in plant communities subjected to habitat loss. Diversity and Distributions, (18):898-908.

Martin, R. and Schlüter, M. (2015). Combining system dynamics and agent-based modeling to analyze social-ecological interactions-an example from modeling restoration of a shallow lake. Frontiers in Environmental Science, 3.

Matter, S. F., Brzyski, J. R., Harrison, C. J., Hyams, S., Loo, C., Loomis, J., Lubbers, H. R., Seastrum, L., Stamper, T. I., Stein, A. M., Stokes, R., and Wilkerson, B. S. (2012). Invading from the garden? A comparison of leaf herbivory for exotic and native plants in natural and ornamental settings. Insect Science, 19(6):677-682.

Matus, G., Papp, M., and Tóthmérész, B. (2005). Impact of management on vegetation dynamics and seed bank formation of inland dune grassland in Hungary. Flora-Morphology, Distribution, Functional Ecology of Plants, 200(3):296-306.

McGill, B. J., Enquist, B. J., Weiher, E., and Westoby, M. (2006). Rebuilding community ecology from functional traits. Trends in Ecology \& Evolution, 21(4):178-185.

McKay, M. D., Beckman, R. J., and Conover, W. J. (1979). A Comparison of Three Methods for Selecting Values of Input Variables in the Analysis of Output From a Computer Code. Technometrics, 42(1):55-61.

Mestre, F., Risk, B. B., Mira, A., Beja, P., and Pita, R. (2017). A metapopulation approach to predict species range shifts under different climate change and landscape connectivity scenarios. Ecological Modelling, 359(Supplement C):406-414. 00001.

Metz, J., Liancourt, P., Kigel, J., Harel, D., Sternberg, M., and Tielbörger, K. (2010). Plant survival in relation to seed size along environmental gradients: a long-term study from semi-arid and Mediterranean annual plant communities. Journal of Ecology, 98(3):697704.

Metz, J., Nisbet, R., and Geritz, S. (1992). How should we define 'fitness' for general ecological scenarios? Trends in Ecology \& Evolution, 7(6):198-202.

Meyerson, L. A. and Mooney, H. A. (2007). Invasive alien species in an era of globalization. Frontiers in Ecology and the Environment, 5(4):199-208.

Mohandass, D., Zhao, J.-L., Xia, Y.-M., Campbell, M. J., and Li, Q.-J. (2015). Increasing temperature causes flowering onset time changes of alpine ginger Roscoea in the Central Himalayas. Journal of Asia-Pacific Biodiversity, 8(3):191-198. 
Moorcroft, P. R., Pacala, S. W., and Lewis, M. A. (2006). Potential role of natural enemies during tree range expansions following climate change. Journal of Theoretical Biology, 241(3):601-616.

Moritz, C. and Agudo, R. (2013). The Future of Species Under Climate Change: Resilience or Decline? Science, 341(6145):504-508.

Morriën, E., Engelkes, T., Macel, M., Meisner, A., and Van der Putten, W. (2010). Climate change and invasion by intracontinental range-expanding exotic plants: the role of biotic interactions. Annals of Botany, 105(6):843.

Morriën, E., Engelkes, T., and van der Putten, W. H. (2011). Additive effects of aboveground polyphagous herbivores and soil feedback in native and range-expanding exotic plants. Ecology, 92(6):1344-1352.

Morris, E. C. (2003). How does fertility of the substrate affect intraspecific competition? Evidence and synthesis from self-thinning. Ecological Research, 18(3):287-305. 00068.

Munch, S. B., Poynor, V., and Arriaza, J. L. (2017). Circumventing structural uncertainty: A Bayesian perspective on nonlinear forecasting for ecology. Ecological Complexity, 32:134-143.

Munson, S. M., Reed, S. C., Peñuelas, J., McDowell, N. G., and Sala, O. E. (2018). Ecosystem thresholds, tipping points, and critical transitions. New Phytologist, 218(4):13151317 .

Nathan, R. (2006). Long-Distance Dispersal of Plants. Science, 313(5788):786-788.

Nathan, R. and Muller-Landau, H. C. (2000). Spatial patterns of seed dispersal, their determinants and consequences for recruitment. Trends in Ecology \& Evolution, 15(7):278285

Nehrbass, N., Winkler, E., Pergl, J., Perglová, I., and Py \vsek, P. (2006). Empirical and virtual investigation of the population dynamics of an alien plant under the constraints of local carrying capacity: Heracleum mantegazzianum in the Czech Republic. Perspectives in Plant Ecology, Evolution and Systematics, 7(4):253-262.

Nicotra, A. B., Atkin, O. K., Bonser, S. P., Davidson, A. M., Finnegan, E. J., Mathesius, U., Poot, P., Purugganan, M. D., Richards, C. L., Valladares, F., and van Kleunen, M. (2010). Plant phenotypic plasticity in a changing climate. Trends in Plant Science, 15(12):684-692.

Nie, M., Zou, J., Xu, X., Liang, C., Fang, C., and Li, B. (2018). Comment on "Unexpected reversal of $\mathrm{C} 3$ versus $\mathrm{C} 4$ grass response to elevated $\mathrm{CO} 2$ during a 20-year field experiment". Science, 361(6405):eaau3016.

Novoplansky, A. (2009). Picking battles wisely: plant behaviour under competition. Plant, Cell \& Environment, 32(6):726-741.

OIff, H. and Ritchie, M. E. (1998). Effects of herbivores on grassland plant diversity. Trends in ecology \& evolution, 13(7):261-265.

Oliver, T. H., Heard, M. S., Isaac, N. J. B., Roy, D. B., Procter, D., Eigenbrod, F., Freckleton, R., Hector, A., Orme, C. D. L., Petchey, O. L., Proença, V., Raffaelli, D., Suttle, K. B., Mace, G. M., Martín-López, B., Woodcock, B. A., and Bullock, J. M. (2015). Biodiversity and Resilience of Ecosystem Functions. Trends in Ecology \& Evolution, 30(11):673-684. 


\section{LITERATURE}

Opdam, P. and Wascher, D. (2004). Climate change meets habitat fragmentation: linking landscape and biogeographical scale levels in research and conservation. Biological Conservation, 117(3):285-297.

Ovaskainen, O. and Hanski, I. (2001). Spatially Structured Metapopulation Models: Global and Local Assessment of Metapopulation Capacity. Theoretical Population Biology, 60(4):281-302.

Paradis, E., Baillie, S. R., and Sutherland, W. J. (2002). Modeling large-scale dispersal distances. Ecological Modelling, 151(2-3):279-292.

Park, I. W. and Mazer, S. J. (2018). Overlooked climate parameters best predict flowering onset: Assessing phenological models using the elastic net. Global Change Biology, 24(12):5972-5984.

Parker, J. D. and Hay, M. E. (2005). Biotic resistance to plant invasions? Native herbivores prefer non-native plants. Ecology Letters, 8(9):959-967.

Parmesan, C. (2006). Ecological and evolutionary responses to recent climate change. Annu. Rev. Ecol. Evol. Syst., 37:637-669.

Parmesan, C. and Hanley, M. E. (2015). Plants and climate change: complexities and surprises. Annals of Botany, 116(6):849-864.

Parmesan, C. and Yohe, G. (2003). A globally coherent fingerprint of climate change impacts across natural systems. Nature, 421(6918):37-42.

Pearson, R. G. and Dawson, T. P. (2003). Predicting the impacts of climate change on the distribution of species: are bioclimate envelope models useful? Global Ecology and Biogeography, 12(5):361-371.

Pedrono, M., Locatelli, B., Ezzine-de Blas, D., Pesche, D., Morand, S., and Binot, A. (2016). Impact of Climate Change on Ecosystem Services. In Torquebiau, E., editor, Climate Change and Agriculture Worldwide, pages 251-261. Springer Netherlands, Dordrecht.

Peel, M. C., Finlayson, B. L., and McMahon, T. A. (2007). Updated world map of the Köppen-Geiger climate classification. Hydrology and Earth System Sciences, 11(5):16331644.

Peltola, H., Kellomäki, S., and Väisänen, H. (1999). Model Computations of the Impact of Climatic Change on the Windthrow Risk of Trees. Climatic Change, 41(1):17-36.

Peñuelas, J. and Boada, M. (2003). A global change-induced biome shift in the Montseny mountains (NE Spain). Global Change Biology, 9(2):131-140.

Pertoldi, C. and Bach, L. A. (2007). Evolutionary aspects of climate-induced changes and the need for multidisciplinarity. Journal of Thermal Biology, 32(3):118-124.

Peters, D. P., Bestelmeyer, B. T., and Turner, M. G. (2007). Cross-scale interactions and changing pattern-process relationships: consequences for system dynamics. Ecosystems, 10(5):790-796.

Peters, D. P., Groffman, P. M., Nadelhoffer, K. J., Grimm, N. B., Collins, S. L., Michener, W. K., and Huston, M. A. (2008). Living in an increasingly connected world: a framework for continental-scale environmental science. Frontiers in Ecology and the Environment, 6(5):229-237. 
Peters, D. P., Pielke, R. A., Bestelmeyer, B. T., Allen, C. D., Munson-McGee, S., and Havstad, K. M. (2004). Cross-scale interactions, nonlinearities, and forecasting catastrophic events. Proceedings of the National Academy of Sciences of the United States of America, 101(42):15130-15135.

Peters, R. H. (1986). The Ecological Implications of Body Size. Cambridge University Press. 05653 Google-Books-ID: OYVxiZgTXWsC.

Phillips, B. (2012). Range shift promotes the formation of stable range edges. Journal of Biogeography.

Pyšek, P., Křivánek, M., and Jarošík, V. (2009). Planting intensity, residence time, and species traits determine invasion success of alien woody species. Ecology, 90(10):27342744.

Pyšek, P. and Richardson, D. M. (2007). Traits Associated with Invasiveness in Alien Plants: Where Do we Stand? In Nentwig, D. W., editor, Biological Invasions, number 193 in Ecological Studies, pages 97-125. Springer Berlin Heidelberg.

Radny, J. and Meyer, K. M. (2018). The role of biotic factors during plant establishment in novel communities assessed with an agent-based simulation model. Technical Report e3088v1, PeerJ Inc.

Radny, J., van der Putten, W. H., Tielbörger, K., and Meyer, K. M. (2018). Influence of seed size on performance of non-native annual plant species in a novel community at two planting densities. Acta Oecologica.

Raffa, K. F., Aukema, B. H., Bentz, B. J., Carroll, A. L., Hicke, J. A., Turner, M. G., and Romme, W. H. (2008). Cross-scale Drivers of Natural Disturbances Prone to Anthropogenic Amplification: The Dynamics of Bark Beetle Eruptions. BioScience, 58(6):501517.

Ramsey, D. S. L., Caley, P. A., and Robley, A. (2015). Estimating population density from presence-absence data using a spatially explicit model. The Journal of Wildlife Management, 79(3):491-499.

Ratajczak, Z., Carpenter, S. R., Ives, A. R., Kucharik, C. J., Ramiadantsoa, T., Stegner, M. A., Williams, J. W., Zhang, J., and Turner, M. G. (2018). Abrupt Change in Ecological Systems: Inference and Diagnosis. Trends in Ecology \& Evolution, 33(7):513526 .

Rehm, E. M., Olivas, P., Stroud, J., and Feeley, K. J. (2015). Losing your edge: climate change and the conservation value of range-edge populations. Ecology and Evolution, 5(19):4315-4326.

Reich, P. B., Hobbie, S. E., and Lee, T. D. (2014). Plant growth enhancement by elevated $\mathrm{CO}_{2}$ eliminated by joint water and nitrogen limitation. Nature Geoscience, 7(12):920924 .

Reich, P. B., Hobbie, S. E., Lee, T. D., and Pastore, M. A. (2018). Unexpected reversal of $\mathrm{C} 3$ versus $\mathrm{C} 4$ grass response to elevated $\mathrm{CO} 2$ during a 20 -year field experiment. Science, 360(6386):317-320.

Richardson, D. M. and Pyšek, P. (2012). Naturalization of introduced plants: ecological drivers of biogeographical patterns. The New Phytologist, 196(2):383-96. 


\section{LITERATURE}

Root, T., Price, J., Hall, K., Schneider, S., Rosenzweig, C., and Pounds, J. (2003). Fingerprints of global warming on wild animals and plants. Nature, 421(6918):57-60.

Roscher, C., Schumacher, J., Baade, J., Wilcke, W., Gleixner, G., Weisser, W. W., Schmid, B., and Schulze, E.-D. (2004). The role of biodiversity for element cycling and trophic interactions: an experimental approach in a grassland community. Basic and Applied Ecology, 5(2):107-121.

Rosenberg, N. J., Blad, B. L., and Verma, S. B. (1983). Microclimate: The Biological Environment. John Wiley \& Sons. Google-Books-ID: c6MI6VvTqTsC.

Rosenfeld, D., Rudich, Y., and Lahav, R. (2001). Desert dust suppressing precipitation: A possible desertification feedback loop. Proceedings of the National Academy of Sciences, 98(11):5975-5980.

Roy, J., Picon-Cochard, C., Augusti, A., Benot, M.-L., Thiery, L., Darsonville, O., Landais, D., Piel, C., Defossez, M., Devidal, S., Escape, C., Ravel, O., Fromin, N., Volaire, F., Milcu, A., Bahn, M., and Soussana, J.-F. (2016). Elevated CO2 maintains grassland net carbon uptake under a future heat and drought extreme. Proceedings of the National Academy of Sciences, 113(22):6224-6229.

Rüegg, J., Gries, C., Bond-Lamberty, B., Bowen, G. J., Felzer, B. S., Mclntyre, N. E., Soranno, P. A., Vanderbilt, K. L., and Weathers, K. C. (2014). Completing the data life cycle: using information management in macrosystems ecology research. Frontiers in Ecology and the Environment, 12(1):24-30.

Ryser, P. (1993). Influences of neighbouring plants on seedling establishment in limestone grassland. Journal of Vegetation Science, 4(2):195-202.

Sagarin, R. D., Gaines, S. D., and Gaylord, B. (2006). Moving beyond assumptions to understand abundance distributions across the ranges of species. Trends in Ecology \& Evolution, 21(9):524-530.

Saikkonen, K., Taulavuori, K., Hyvönen, T., Gundel, P. E., Hamilton, C. E., Vänninen, I., Nissinen, A., and Helander, M. (2012). Climate change-driven species' range shifts filtered by photoperiodism. Nature Climate Change, 2(4):239-242.

Sandel, B. and Smith, A. B. (2009). Scale as a lurking factor: incorporating scaledependence in experimental ecology. Oikos, 118(9):1284-1291.

Saura, S., Bodin, O., and Fortin, M.-J. (2013). Stepping stones are crucial for species' long-distance dispersal and range expansion through habitat networks.

Saura, S., Bodin, O., and Fortin, M.-J. (2014). Stepping stones are crucial for species' long-distance dispersal and range expansion through habitat networks. Journal of Applied Ecology, 51(1):171-182. WOS:000329846500019.

Scheepens, J. F. and Stöcklin, J. (2013). Flowering phenology and reproductive fitness along a mountain slope: maladaptive responses to transplantation to a warmer climate in Campanula thyrsoides. Oecologia, 171(3):679-691.

Scheffers, B. R., Meester, L. D., Bridge, T. C. L., Hoffmann, A. A., Pandolfi, J. M., Corlett, R. T., Butchart, S. H. M., Pearce-Kelly, P., Kovacs, K. M., Dudgeon, D., Pacifici, M., Rondinini, C., Foden, W. B., Martin, T. G., Mora, C., Bickford, D., and Watson, J. E. M. (2016). The broad footprint of climate change from genes to biomes to people. Science, 354(6313):aaf7671. 
Schiffers, K. and Tielboerger, K. (2006). Ontogenetic shifts in interactions among annual plants. Journal of Ecology, 94(2):336-341.

Schluter, D. (2001). Ecology and the origin of species. Trends in Ecology \& Evolution, 16(7):372-380.

Schmitt, J., Niles, J., and Wulff, R. D. (1992). Norms of Reaction of Seed Traits to Maternal Environments in Plantago lanceolata. The American Naturalist, 139(3):451-466.

Schmitz, O. (1997). Press pertubations and the predictability of ecological interactions in a food web. Ecology, 78(1):55-69.

Schmitz, O. J. (2000). Combining field experiments and individual-based modeling to identify the dynamically relevant organizational scale in a field system. Oikos, 89(3):471484 .

Schneider, D. C. (2001). The Rise of the Concept of Scale in Ecology The concept of scale is evolving from verbal expression to quantitative expression. BioScience, 51(7):545-553.

Schneider, M. K., Law, R., and Illian, J. B. (2006). Quantification of neighbourhooddependent plant growth by Bayesian hierarchical modelling. Journal of Ecology, 94(2):310-321.

Schuur, E. A. G. and Abbott, B. (2011). Climate change: High risk of permafrost thaw. Nature, 480:32-33.

Schweiger, O., Settele, J., Kudrna, O., Klotz, S., and Kühn, I. (2008). Climate Change Can Cause Spatial Mismatch of Trophically Interacting Species. Ecology, 89(12):3472-3479.

Schwinning, S. (1996). Decomposition analysis of competitive symmetry and size structure dynamics. Annals of Botany, 77(1):47-58.

Schwinning, S. and Weiner, J. (1998). Mechanisms determining the degree of size asymmetry in competition among plants. Oecologia, 113(4):447-455.

Scott, P. A., Lavoie, C., MacDonald, G. M., Sveinbjörnsson, B., and Wein, R. W. (1997). Climate Change and Future Position of Arctic Tree Line. In Oechel, W. C., Callaghan, T. V., Gilmanov, T. G., Holten, J. I., Maxwell, B., Molau, U., and Sveinbjörnsson, B., editors, Global Change and Arctic Terrestrial Ecosystems, Ecological Studies, pages 245-265. Springer New York, New York, NY.

Sepulveda-Jauregui, A., Hoyos-Santillan, J., Martinez-Cruz, K., Walter Anthony, K. M., Casper, P., Belmonte-Izquierdo, Y., and Thalasso, F. (2018). Eutrophication exacerbates the impact of climate warming on lake methane emission. Science of The Total Environment, 636:411-419.

Sherry, R. A., Zhou, X., Gu, S., Arnone, J. A., Schimel, D. S., Verburg, P. S., Wallace, L. L., and Luo, Y. (2007). Divergence of reproductive phenology under climate warming. Proceedings of the National Academy of Sciences, 104(1):198-202.

Shi, T., Huang, Y., Wang, H., Shi, C.-E., and Yang, Y.-J. (2015). Influence of urbanization on the thermal environment of meteorological station: Satellite-observed evidence. Advances in Climate Change Research, 6(1):7-15.

Shimada, K., Kamoshida, T., Itoh, M., Nishino, S., Carmack, E., McLaughlin, F., Zimmermann, S., and Proshutinsky, A. (2006). Pacific Ocean inflow: Influence on catastrophic reduction of sea ice cover in the Arctic Ocean. Geophysical Research Letters, 33(8). 


\section{LITERATURE}

Silvertown, J. and Charlesworth, D. (2001). Introduction to plant population biology. John Wiley \& Sons.

Simberloff, D. (2009). The Role of Propagule Pressure in Biological Invasions. Annual Review of Ecology, Evolution, and Systematics, 40(1):81-102. 00749.

Simpson, S. J. and Abisgold, J. D. (1985). Compensation by locusts for changes in dietary nutrients: behavioural mechanisms. Physiological Entomology, 10(4):443-452.

Singer, A., Johst, K., Banitz, T., Fowler, M. S., Groeneveld, J., Gutiérrez, A. G., Hartig, F., Krug, R. M., Liess, M., Matlack, G., Meyer, K. M., Pe'er, G., Radchuk, V., VoinopolSassu, A.-J., and Travis, J. M. J. (2016). Community dynamics under environmental change: How can next generation mechanistic models improve projections of species distributions? Ecological Modelling, 326:63-74.

Smith, D. W., Peterson, R. O., and Houston, D. B. (2003). Yellowstone after Wolves. BioScience, 53(4):330-340.

Soliveres, S., Maestre, F. T., Bowker, M. A., Torices, R., Quero, J. L., García-Gómez, M., Cabrera, O., Cea, A., Coaguila, D., Eldridge, D. J., Espinosa, C. I., Hemmings, F., Monerris, J. J., Tighe, M., Delgado-Baquerizo, M., Escolar, C., García-Palacios, P., Gozalo, B., Ochoa, V., Blones, J., Derak, M., Ghiloufi, W., Gutiérrez, J. R., Hernández, R. M., and Noumi, Z. (2014). Functional traits determine plant co-occurrence more than environment or evolutionary relatedness in global drylands. Perspectives in plant ecology, evolution and systematics, 16(4):164-173.

Soons, M. B. and Ozinga, W. A. (2005). How important is long-distance seed dispersal for the regional survival of plant species? Diversity and Distributions, 11(2):165-172.

Soranno, P. A., Cheruvelii, K. S., Bissell, E. G., Bremigan, M. T., Downing, J. A., Fergus, C. E., Filstrup, C. T., Henry, E. N., Lottig, N. R., Stanley, E. H., Stow, C. A., Tan, P.-N., Wagner, T., and Webster, K. E. (2014). Cross-scale interactions: quantifying multi-scaled cause-effect relationships in macrosystems. Frontiers in Ecology and the Environment, 12(1):65-73.

Soranno, P. A. and Schimel, D. S. (2014). Macrosystems ecology: big data, big ecology. Frontiers in Ecology and the Environment, 12(1):3-3.

Speek, T. A. A., Lotz, L. A. P., Ozinga, W. A., Tamis, W. L. M., Schaminée, J. H. J., and van der Putten, W. H. (2011). Factors relating to regional and local success of exotic plant species in their new range. Diversity and Distributions, 17(3):542-551.

Staniczenko, P. P. A., Sivasubramaniam, P., Suttle, K. B., and Pearson, R. G. (2017). Linking macroecology and community ecology: refining predictions of species distributions using biotic interaction networks. Ecology Letters, 20(6):693-707.

Stanton-Geddes, J., Tiffin, P., and Shaw, R. (2012). Role of climate and competitors in limiting fitness across range edges of an annual plant. Ecology, 93(7):1604-1613.

Steffen, W., Rockström, J., Richardson, K., Lenton, T. M., Folke, C., Liverman, D., Summerhayes, C. P., Barnosky, A. D., Cornell, S. E., Crucifix, M., Donges, J. F., Fetzer, I., Lade, S. J., Scheffer, M., Winkelmann, R., and Schellnhuber, H. J. (2018). Trajectories of the Earth System in the Anthropocene. Proceedings of the National Academy of Sciences, 115(33):8252-8259.

Steidl, R. J. and Thomas, L. (2001). Power analysis and experimental design. Design and analysis of ecological experiments. Oxford University Press, Oxford, pages 14-36. 
Straka, J. R. and Starzomski, B. M. (2014). Humming along or buzzing off? The elusive consequences of plant-pollinator mismatches. Journal of Pollination Ecology, 13(0).

Strauss, S. Y. and Agrawal, A. A. (1999). The ecology and evolution of plant tolerance to herbivory. Trends in Ecology \& Evolution, 14(5):179-185.

Stukonis, V. and Slepetys, J. (2013). The Assessment of Agrostis Capillaris Wild Populations for Use in Turf Grass Breeding. Proceedings of the Latvian Academy of Sciences. Section B. Natural, Exact, and Applied Sciences., 67(3):277-280.

Svenning, J.-C., Gravel, D., Holt, R. D., Schurr, F. M., Thuiller, W., Münkemüller, T., Schiffers, K. H., Dullinger, S., Edwards, T. C., Hickler, T., and others (2014). The influence of interspecific interactions on species range expansion rates. Ecography, 37(12):1198-1209.

Talluto, M. V., Boulangeat, I., Ameztegui, A., Aubin, I., Berteaux, D., Butler, A., Doyon, F., Drever, C. R., Fortin, M.-J., Franceschini, T., Liénard, J., McKenney, D., Solarik, K. A., Strigul, N., Thuiller, W., and Gravel, D. (2016). Cross-scale integration of knowledge for predicting species ranges: a metamodelling framework. Global Ecology and Biogeography, 25(2):238-249.

Tamis, W., Zelfde, M., Meijden, R., and Haes, H. (2005). Changes in vascular plant biodiversity in the Netherlands in the 20th century explained by their climatic and other environmental characteristics. Climatic Change, 72(1):37-56.

Taylor, K. T., Maxwell, B. D., Pauchard, A., Nuñez, M. A., Peltzer, D. A., Terwei, A., and Rew, L. J. (2016). Drivers of plant invasion vary globally: evidence from pine invasions within six ecoregions. Global Ecology and Biogeography, 25(1):96-106.

Team, R. D. C. (2016). R: A Language and Environment for Statistical Computing. ISBN 3-900051-07-0.

Temperli, C., Bugmann, H., and Elkin, C. (2013). Cross-scale interactions among bark beetles, climate change, and wind disturbances: a landscape modeling approach. Ecological Monographs, 83(3):383-402.

Thomas, C. D. (2010). Climate, climate change and range boundaries. Diversity and Distributions, 16(3):488-495.

Thomas, C. D., Cameron, A., Green, R. E., Bakkenes, M., Beaumont, L. J., Collingham, Y. C., Erasmus, B. F. N., de Siqueira, M. F., Grainger, A., Hannah, L., Hughes, L., Huntley, B., van Jaarsveld, A. S., Midgley, G. F., Miles, L., Ortega-Huerta, M. A., Townsend Peterson, A., Phillips, O. L., and Williams, S. E. (2004). Extinction risk from climate change. Nature, 427(6970):145-148.

Thompson, P. L., Rayfield, B., and Gonzalez, A. (2017). Loss of habitat and connectivity erodes species diversity, ecosystem functioning, and stability in metacommunity networks. Ecography, 40(1):98-108.

Thuiller, W., Gassó, N., Pino, J., and Vila, M. (2012). Ecological niche and species traits: key drivers of regional plant invader assemblages. Biological Invasions, pages 1-18.

Thuiller, W., Lavorel, S., Araújo, M., Sykes, M., and Prentice, I. (2005). Climate change threats to plant diversity in Europe. Proceedings of the National Academy of Sciences of the United States of America, 102(23):8245. 


\section{LITERATURE}

Tomiolo, S., Putten, W. H. v. d., and Tielbörger, K. (2015). Separating the role of biotic interactions and climate in determining adaptive response of plants to climate change. Ecology, 96(5):1298-1308.

Tracey, A. J. and Aarssen, L. W. (2014). Revising traditional theory on the link between plant body size and fitness under competition: evidence from old-field vegetation. Ecology and Evolution, 4(7):959-967.

Travis, J., Harris, C., Park, K., and Bullock, J. (2011). Improving prediction and management of range expansions by combining analytical and individual-based modelling approaches. Methods in Ecology and Evolution, (2):477-488.

Tremlová, K. and Münzbergová, Z. (2007). Importance of species traits for species distribution in fragmented landscapes. Ecology, 88(4):965-977.

Turco, M., Llasat, M.-C., Hardenberg, J. v., and Provenzale, A. (2014). Climate change impacts on wildfires in a Mediterranean environment. Climatic Change, 125(3-4):369380.

Tyberghein, L., Verbruggen, H., Pauly, K., Troupin, C., Mineur, F., and De Clerck, O. (2012). Bio-ORACLE: a global environmental dataset for marine species distribution modelling. Global Ecology and Biogeography, 21(2):272-281.

Ummenhofer, C. C. and Meehl, G. A. (2017). Extreme weather and climate events with ecological relevance: a review. Philosophical Transactions of the Royal Society B: Biological Sciences, 372(1723):20160135.

UN (2015). CHAPTER XXVII 7. d: Paris Agreement.

Urban, M. C. (2015). Accelerating extinction risk from climate change. Science, 348(6234):571-573.

Urban, M. C., Zarnetske, P. L., and Skelly, D. K. (2013). Moving forward: dispersal and species interactions determine biotic responses to climate change. Annals of the New York Academy of Sciences, 1297(1):44-60.

Van der Putten, W. H. (2012). Climate Change, Aboveground-Belowground Interactions, and Species' Range Shifts. Annual Review of Ecology, Evolution, and Systematics, 43(1):365-383.

van der Putten, W. H., Bardgett, R. D., de Ruiter, P. C., Hol, W. H. G., Meyer, K. M., Bezemer, T. M., Bradford, M. A., Christensen, S., Eppinga, M. B., Fukami, T., Hemerik, L., Molofsky, J., Schädler, M., Scherber, C., Strauss, S. Y., Vos, M., and Wardle, D. A. (2009). Empirical and theoretical challenges in aboveground-belowground ecology. Oecologia, 161(1):1-14.

van der Putten, W. H., de Ruiter, P. C., Martijn Bezemer, T., Harvey, J. A., Wassen, M., and Wolters, V. (2004). Trophic interactions in a changing world. Basic and Applied Ecology, 5(6):487-494.

Van Der Putten, W. H., Macel, M., and Visser, M. E. (2010). Predicting species distribution and abundance responses to climate change: why it is essential to include biotic interactions across trophic levels. Philosophical Transactions of the Royal Society B: Biological Sciences, 365(1549):2025-2034. 
Van Grunsven, R. H. A., Van der Putten, W. H., Bezemer, T. M., Berendse, F., and Veenendaal, E. M. (2010). Plant-soil interactions in the expansion and native range of a poleward shifting plant species. Global Change Biology, 16(1):380-385.

Vilà, M. and Weiner, J. (2004). Are invasive plant species better competitors than native plant species? - evidence from pair-wise experiments. Oikos, 105(2):229-238.

Vincenot, C. E., Giannino, F., Rietkerk, M., Moriya, K., and Mazzoleni, S. (2011). Theoretical considerations on the combined use of System Dynamics and individual-based modeling in ecology. Ecological Modelling, 222(1):210-218.

Violle, C., Navas, M., Vile, D., Kazakou, E., Fortunel, C., Hummel, I., and Garnier, E. (2007). Let the concept of trait be functional! Oikos, 116(5):882-892.

Vitt, P., Belmaric, P. N., Book, R., and Curran, M. (2016). Assisted migration as a climate change adaptation strategy: lessons from restoration and plant reintroductions. Israel Journal of Plant Sciences, 63(4):250-261.

Walther, G., Berger, S., and Sykes, M. (2005). An ecological 'footprint' of climate change. Proceedings of the Royal Society B: Biological Sciences, 272(1571):1427-1432.

Walther, G., Post, E., Convey, P., Menzel, A., Parmesan, C., Beebee, T., Fromentin, J., Hoegh-Guldberg, O., and Bairlein, F. (2002). Ecological responses to recent climate change. Nature, 416(6879):389-395.

Walther, G.-R., Roques, A., Hulme, P. E., Sykes, M. T., Pyšek, P., Kühn, I., Zobel, M., Bacher, S., Botta-Dukát, Z., Bugmann, H., Czúcz, B., Dauber, J., Hickler, T., Jarošík, V., Kenis, M., Klotz, S., Minchin, D., Moora, M., Nentwig, W., Ott, J., Panov, V. E., Reineking, B., Robinet, C., Semenchenko, V., Solarz, W., Thuiller, W., Vilà, M., Vohland, K., and Settele, J. (2009). Alien species in a warmer world: risks and opportunities. Trends in Ecology \& Evolution, 24(12):686-693.

War, A. R., Paulraj, M. G., Ahmad, T., Buhroo, A. A., Hussain, B., Ignacimuthu, S., and Sharma, H. C. (2012). Mechanisms of plant defense against insect herbivores. Plant Signaling \& Behavior, 7(10):1306-1320.

Warren, R. J., Bahn, V., and Bradford, M. A. (2012). The interaction between propagule pressure, habitat suitability and density-dependent reproduction in species invasion. Oikos, 121(6):874-881.

Weigelt, A., Marquard, E., Temperton, V. M., Roscher, C., Scherber, C., Mwangi, P. N., Felten, S., Buchmann, N., Schmid, B., Schulze, E.-D., and Weisser, W. W. (2010). The Jena Experiment: six years of data from a grassland biodiversity experiment. Ecology, 91(3):930-931.

Weiner, J. and Damgaard, C. (2006). Size-asymmetric competition and size-asymmetric growth in a spatially explicit zone-of-influence model of plant competition. Ecological Research, 21(5):707-712. WOS:000240282900011.

Weiner, J., Stoll, P., Muller-Landau, H., and Jasentuliyana, A. (2001). The Effects of Density, Spatial Pattern, and Competitive Symmetry on Size Variation in Simulated Plant Populations. The American Naturalist, 158(4):438-450. ArticleType: researcharticle / Full publication date: October 2001 / Copyright (C) 2001 The University of Chicago. 


\section{LITERATURE}

Westoby, M., Leishman, M., Lord, J., Poorter, H., Schoen, D., Westoby, M., Leishman, M., Lord, J., Poorter, H., and Schoen, D. (1996). Comparative ecology of seed size and dispersal [and discussion]. Philosophical Transactions of the Royal Society of London. Series B: Biological Sciences, 351(1345):1309-1318.

Wickham, H. (2009). ggplot2: Elegant Graphics for Data Analysis. Use R! Springer-Verlag, New York.

Wiegand, K., Saltz, D., Ward, D., and Levin, S. A. (2008). The role of size inequality in selfthinning: A pattern-oriented simulation model for arid savannas. Ecological Modelling, 210(4):431-445.

Wiegand, K., Schmidt, H., Jeltsch, F., and Ward, D. (2000). Linking a spatially-explicit model of acacias to GIS and remotely-sensed data. Folia Geobotanica, 35(2):211-230.

Wikle, C. K. and Hooten, M. B. (2006). Hierarchical Bayesian spatio-temporal models for population spread. Applications of computational statistics in the environmental sciences: hierarchical Bayes and MCMC methods, pages 145-169.

Williams, J. L., Kendall, B. E., and Levine, J. M. (2016). Rapid evolution accelerates plant population spread in fragmented experimental landscapes. Science, 353(6298):482-485.

Williams, J. W. and Jackson, S. T. (2007). Novel climates, no-analog communities, and ecological surprises. Frontiers in Ecology and the Environment, 5(9):475-482.

Williams, J. W., Jackson, S. T., and Kutzbach, J. E. (2007). Projected distributions of novel and disappearing climates by 2100 AD. Proceedings of the National Academy of Sciences, 104(14):5738-5742.

Wisz, M. S., Pottier, J., Kissling, W. D., Pellissier, L., Lenoir, J., Damgaard, C. F., Dormann, C. F., Forchhammer, M. C., Grytnes, J.-A., Guisan, A., Heikkinen, R. K., Høye, T. T., Kühn, I., Luoto, M., Maiorano, L., Nilsson, M.-C., Normand, S., Ockinger, E., Schmidt, N. M., Termansen, M., Timmermann, A., Wardle, D. A., Aastrup, P., and Svenning, J.-C. (2013). The role of biotic interactions in shaping distributions and realised assemblages of species: implications for species distribution modelling. Biological reviews of the Cambridge Philosophical Society, 88(1):15-30.

Wittenberg, R., Kenis, M., Blick, T., Hänggi, A., Gassmann, A., Weber, E., et al. (2006). Invasive alien species in Switzerland: an inventory of alien species and their threat to biodiversity and economy in Switzerland. Technical report, CABI Bioscience Switzerland Centre.

WMO (1966). International Meteorological Vocabulary. Number 182 in WMO / WMO. Geneva : Secretariat of the WMO, 1951- ; 182. Secretariat of the World Meteorological Organization.

Wu, J. (1999). Hierarchy and Scaling: Extrapolating Information along a Scaling Ladder. Canadian Journal of Remote Sensing, 25(4):367-380.

Wu, J. and David, J. L. (2002). A spatially explicit hierarchical approach to modeling complex ecological systems: theory and applications. Ecological Modelling, 153(1):726.

Yeh, S.-W., Kug, J.-S., Dewitte, B., Kwon, M.-H., Kirtman, B. P., and Jin, F.-F. (2009). El Niño in a changing climate. Nature, 461(7263):511-514. 
Yuan, S., Tan, P., Cheruvelil, K. S., Collins, S. M., and Soranno, P. A. (2015). Constrained spectral clustering for regionalization: Exploring the trade-off between spatial contiguity and landscape homogeneity. In 2015 IEEE International Conference on Data Science and Advanced Analytics (DSAA), pages 1-10.

Zakharova, L., Meyer, K. M., and Seifan, M. (2019). Trait-based modelling in ecology: lessons from two decades of research. Technical Report e27484v1, PeerJ Inc.

Zenni, R. D., Bailey, J. K., and Simberloff, D. (2014). Rapid evolution and range expansion of an invasive plant are driven by provenance-environment interactions. Ecology Letters, pages $n / a-n / a$.

Zenni, R. D. and Nunez, M. A. (2013). The elephant in the room: the role of failed invasions in understanding invasion biology. Oikos, 122(6):801-815. WOS:000319408300001.

Zhang, Y., Loreau, M., He, N., Wang, J., Pan, Q., Bai, Y., and Han, X. (2018). Climate variability decreases species richness and community stability in a temperate grassland. Oecologia, 188(1):183-192.

Zhu, K., Woodall, C. W., and Clark, J. S. (2012). Failure to migrate: lack of tree range expansion in response to climate change. Global Change Biology, 18(3):1042-1052.

Zillio, T. and He, F. (2010). Inferring species abundance distribution across spatial scales. Oikos, 119(1):71-80. 

Appendix 



\section{A. Supplemental Material: Alternative herbivory simulations}

The content of this chapter was originally published as supplemental material for Radny and Meyer (2018).

\section{Motivation}

In the standard version of our simulation model, herbivores consume an absolute amount of biomass per plantof $7.4-7.6 \mathrm{mg}$. This might lead to relatively higher losses in terms of percentage foliage of small plants compared to lager plants.

To investigate whether and how this affects model results, we adjusted the analysis for this effect by implementing herbivore consumption as relative amounts of plant biomass in an alternative model version.

\section{Methods}

In the alternative model version, each herbivore consumes between $1.2 \%$ and $4.5 \%$ of the total current biomass of a plant that it successfully attacked. The mean of these percentages corresponds to the percentage of the mean biomass of all plants in a typical simulation that is represented by $7.5 \mathrm{mg}$.

NetLogo Code snippet from the standard version:

set biomass_now biomass_now $-((7.5 *(1+$ random-float 0.1$)) *$

count herbivores-here) 
NetLogo Code snippet from the alternative version:

set biomass_now biomass_now $*$ ( (0.988 -(random-float $0.033 *$

count herbivores-here)))

The alternative model version was run and analyzed in the same way as the standard model version(see section 3.2.12 in chapter 3). We plotted graphs corresponding to figs. 3.5 to 3.8 in chapter 3.

\section{Results}

We found qualitatively similar results for standard and alternative model versions (figs. A.1 to A.4). The main difference was that the effect of high competitiveness traits on survival was much less pronounced in the alternative than in the standard version. Furthermore, the effect of high defense traits on survival was more visible (or at all detectable) in the alternative than in the standard model version.

\section{Conclusions}

We conclude that the qualitative results of our model are robust towards different herbivory implementations. The quantitative differences indicate, though, that it would be worthwhile to explore a greater range and resolution of herbivory implementations in the future.

These will require more data for parameterization than arecurrently available. Thus, concerted empirical-modelling efforts are called for. 


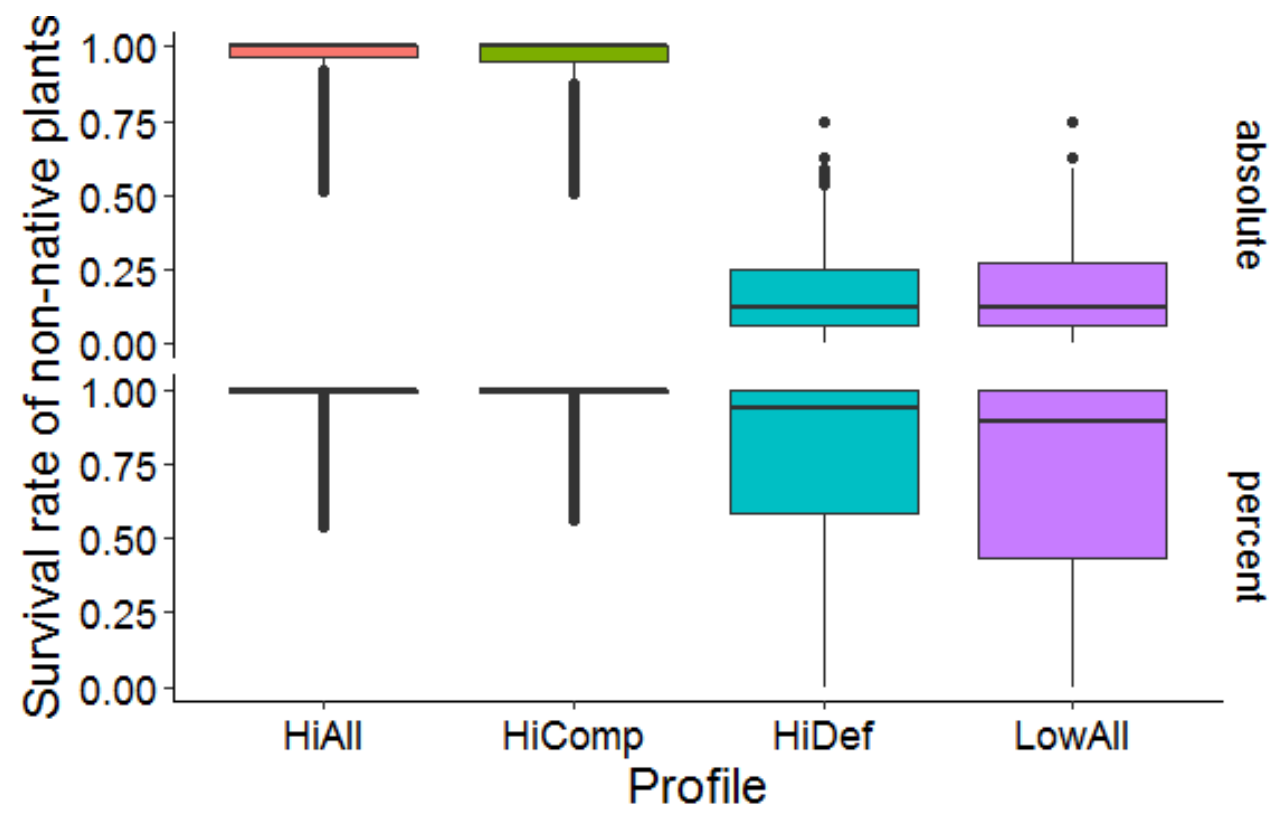

Figure A.1. - Alternative run: Survival rates of non-native plants with different trait profiles for the standard (absolute) and alternative (percent) model version. The trait profiles are: high competitive and high defensive (HiAll), high competitive and low defensive (HiComp), low competitive and high defensive (HiDef), and low competitive and low defensive (LowAll). Values were averaged over all scenarios.

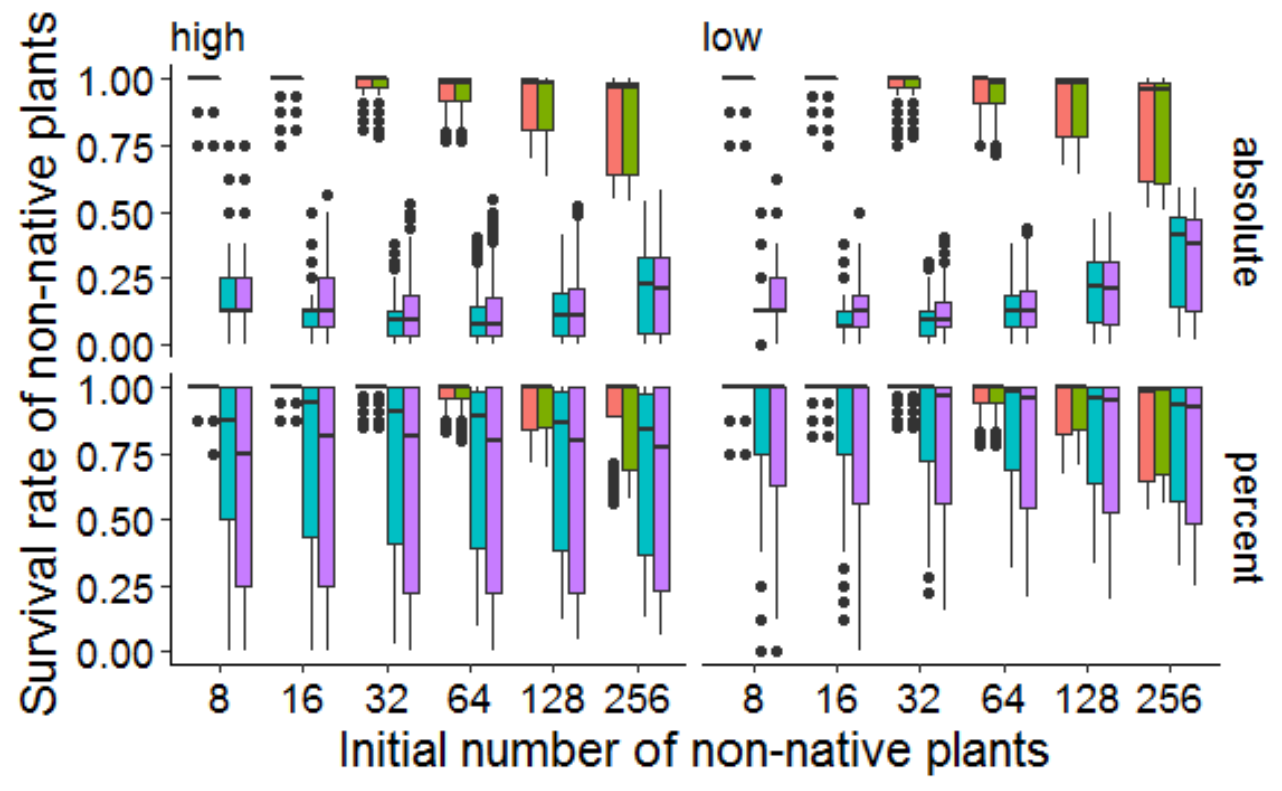

Figure A.2. - Alternative run: Survival rates of non-native plants under different invasion levels in high density (left panels) and low density (right panels) native communities for the standard (absolute) and alternative (percent) model version. Invasion level corresponds to initial population sizes of $8,16,32,64,128$ and 256 individuals of the non-native species. Non-natives are split into the following trait profiles (in each block from left to right): high competitive and high defensive (HiAll, orange), high competitive and low defensive (HiComp, green), low competitive and high defensive (HiDef, blue), and low competitive and low defensive (LowAll, purple). 


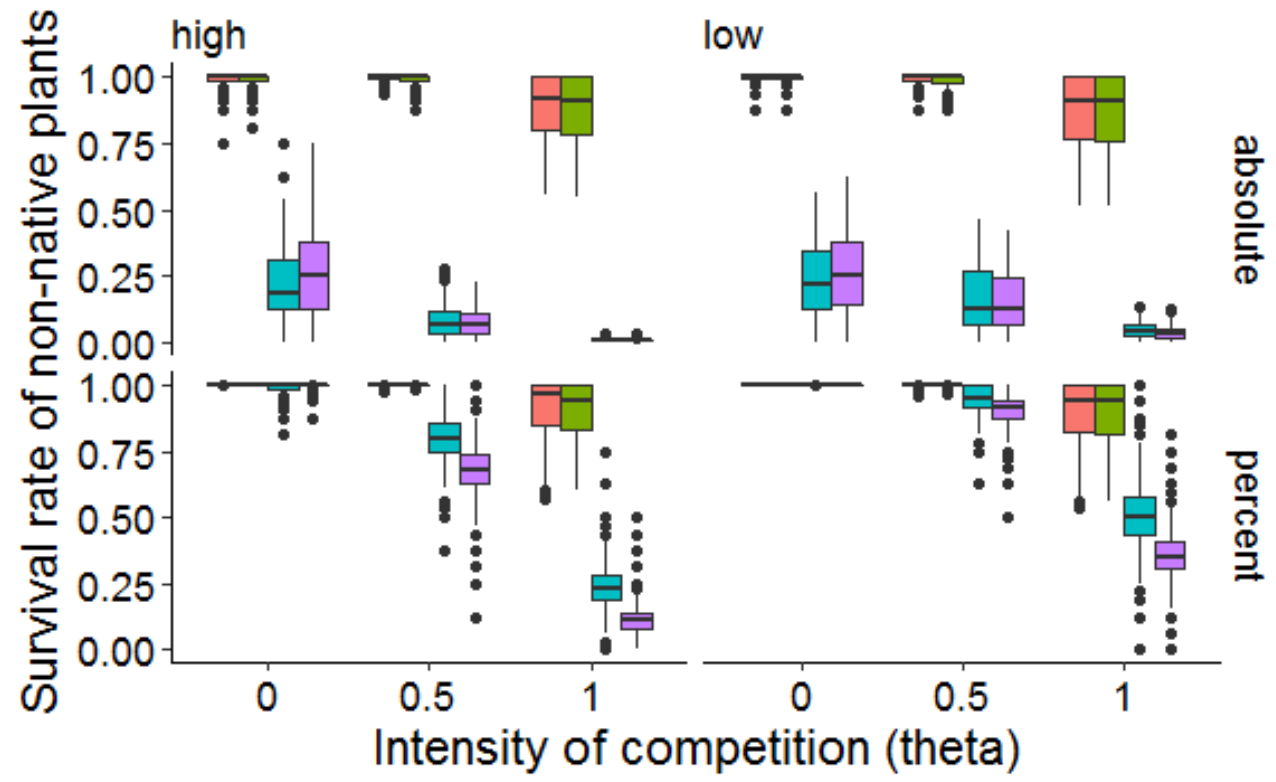

(a) Even mixture of natives in the initial community

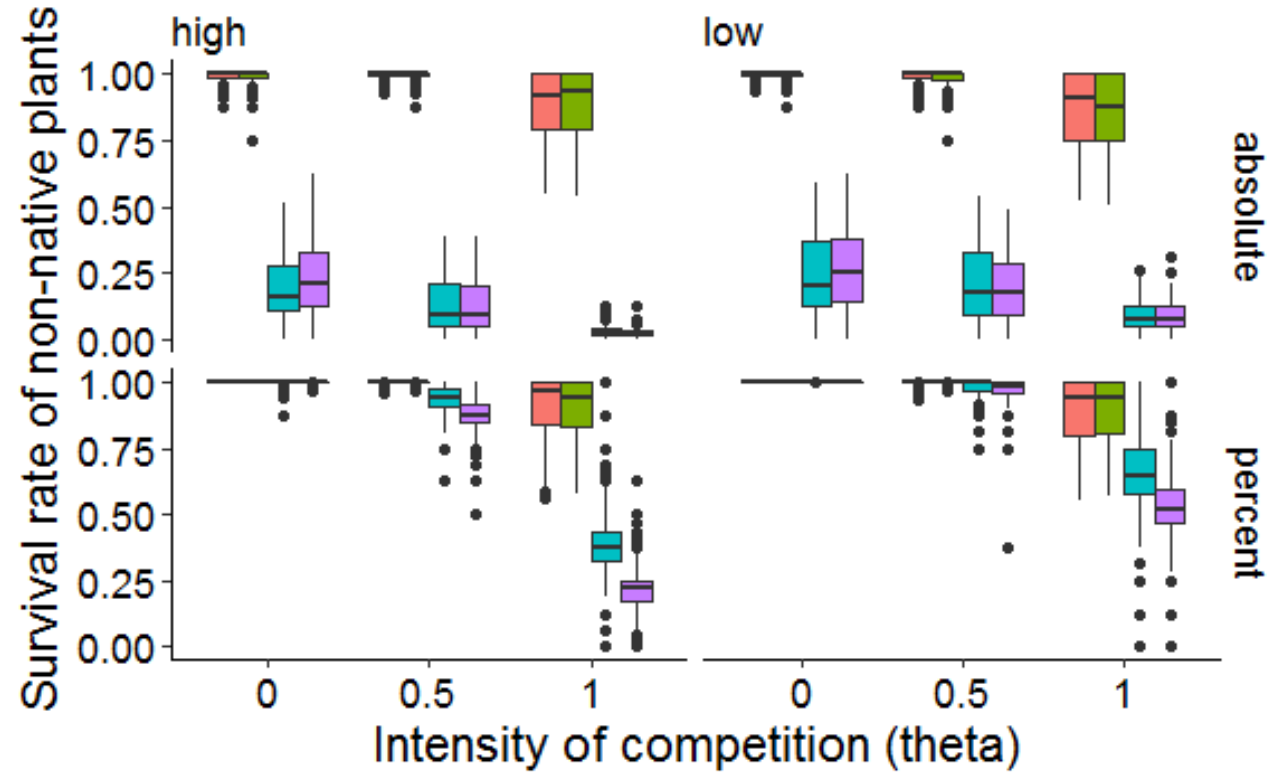

(b) Native species mixture based on literature values of seed bank sizes

Figure A.3. - Alternative run: Effect of the intensity of competition $\Theta$ on survival rates of nonnative plants in different configurations of the resident community for the standard (absolute) and alternative (percent) model version. Left panels show high density of natives, right panels show low density of natives. Note that different initial numbers of non-natives are not separated in this figure. The upper four (fig. A.3a) panels show an even mixture of natives in the initial community, the lower four panels (fig. A.3b) show a mixture based on literature values of seed bank sizes. With $\Theta=0$, resources are shared among competitors regardless of their biomass, with $\Theta=1$, resources are shared proportionally to the biomass of the individual competitors. $\Theta=0.5$ reflects an intermediate stage.

Non-natives are split into the following trait profiles (in each block from left to right): high competitive and high defensive (HiAll, orange), high competitive and low defensive (HiComp, green), low competitive and high defensive (HiDef, blue), and low competitive and low defensive (LowAll, purple). 


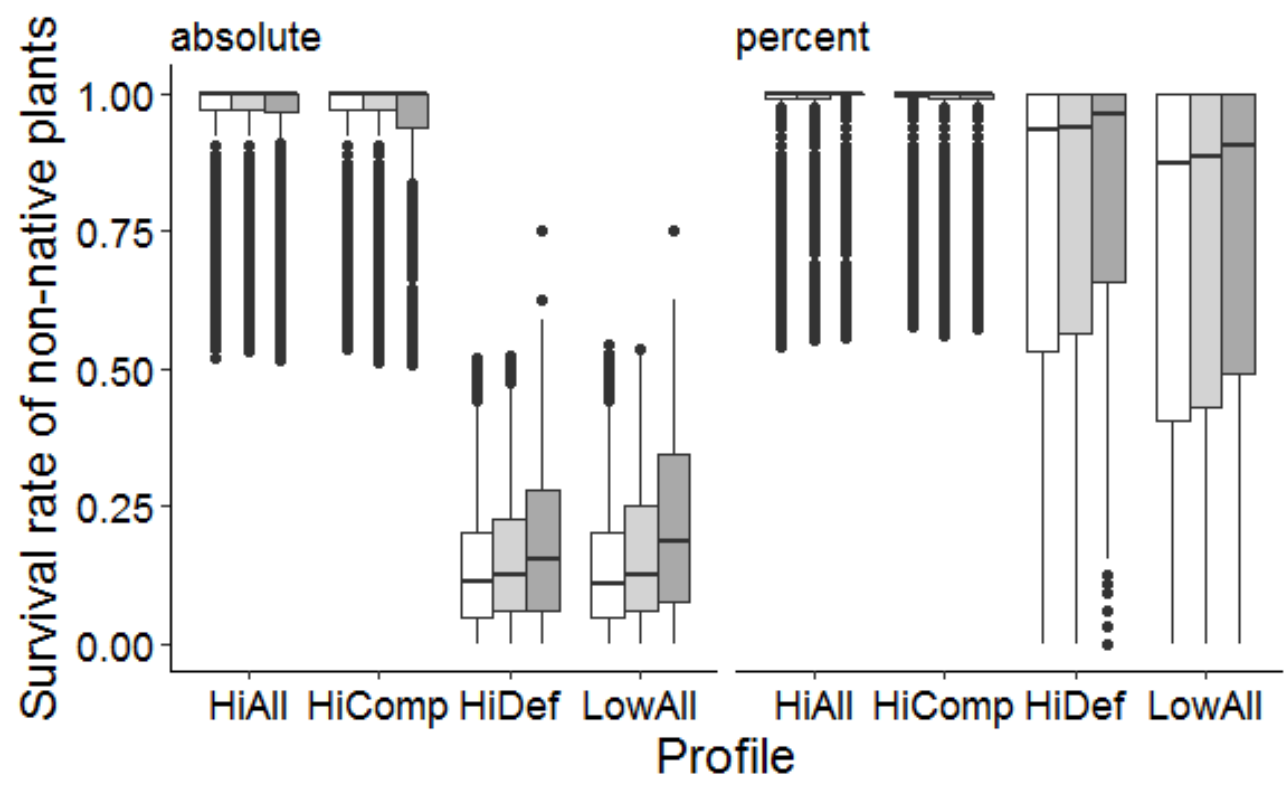

Figure A.4. - Alternative run: Survival rate of non-native species at different herbivore densities for the standard (absolute) and alternative (percent) model version. Herbivore densities: no herbivores as control scenario (white bars), 3.2 herbivores per $m^{2}$ (light grey bars), and 18.75 herbivores per $m^{2}$ (dark grey bars). Non-natives are split into the following trait profiles: high competitive and high defensive (HiAll), high competitive and low defensive (HiComp), low competitive and high defensive (HiDef), and low competitive and low defensive (LowAll). 



\title{
Acknowledgements
}

\author{
Wer über gewisse Dinge den \\ Verstand nicht verliert, der hat \\ keinen zu verlieren.
}

Gotthold Ephraim Lessing

Throughout this thesis, I was lucky to be supported and accompanied by many great people. They shared scientific and professional advice as well as emotional support and guidance in many decisions.

First of all, I want to thank Katrin Meyer. You have the rare gift to encourage and motivate people far beyond what they had thought to be able to achieve. Especially during the constant cycle of submission, revision, rejection and starting all over again with the experimental paper, you were the one who always saw the silver lining. If not for you, I do not know whether this thesis would have been ever finished.

Kerstin Wiegand collected an amazing group of scientists and created an incredibly inspiring atmosphere in our department. One of her strenghts was to treat all of us as equal scientists, taking our ideas serious and allowed us to stand on our own scientific feet. Besides being always comitted to have the department, the GRK 1644 , and so many more projects running and humming, she also found the time for enjoying the world beyond science with us, with or without broomsticks.

I thank the ecosystem modelling team for their valuable feedback many times, the amazing atmosphere for serious and sometimes not so serious work in the ball room and the satelite population office. After I left Göttingen in 2015, Sebastian Hanß and Frauke Thorade always had a niche for me when I stopped over. 
Ilona Watteler-Spang was the good spirit for our entire team. Her angelic patience and willingness to find solutions made working at the University of Göttingen even more enjoyable.

Being part of the GRK 1644 "Scaling Problems in Statistics" was a highly inspring and valuable experience for me. Here I learned that it is always worth a look into other disciplines, no matter how far away they seem - in the end, you'll find shared problems and in many cases new inspiration on how to solve them. I am very happy to have made this experience in highly interdisciplinary work - and I share that with my own students now. I would like to give a special thank to Thomas Kneibb and Barbara Strauss for their patience and sympathy with me and all of the students in the GRK 1644.

The experiment has been carried out with the help of great people from the NIOO-KNAW and the University of Wageningen. First of all, Wim van der Putten was an amazing partner to collaborate with, always finding the kindest words for critique and advice.

During the actual experiment, I was supported by several good spirits: Jasper van Ruijven provided us with seeds of native species, Jeff Harvey and Daniela Weber shared their Mamestra caterpillars with us and gave helpful advice. During preparation and execution of the experiment, Freddy ten Hooven, Rebecca Pas, Roel Wagenaar, Gregor Disveld and Ciska Raaijmakers provided great help. Seeds of non-native species were collected in situ by Sara Tomiolo in collaboration and with advice from Katja Tielbörger of the University of Tübingen.

There is a world beyond science and also there I experienced support when I needed strength and the necessary distraction when I got stuck.

I want to thank my parents and family, who helped and supported me in many more ways than I can count, not only during the doctoral studies, but way before that. They opened doors for all of us three kids, repeatedly when necessary, and gave us the courage to stand in for our desicions and opinions. Mama, I'm sorry we don't have the tradition of throwing of the hat here, but hopefully kissing the Gänseliesel will be an adequate substitute.

Of course many friends were with me during these years. Representative of all, I want to thank especially three people.

Toni Tittel, chitchat nights in the Black Forest with wine and campfires are the those 
shiny gems that let you lay back and know life is good. By the way, throughout this thesis, you sent me more than 130 postcards to four different adresses!

Christian Schutter, you lifted me up in the truest sense and made losing the ground under my feet the best feeling in the world.

Simon Gewölb, no matter how far away, kind words light up each day and the many work-through nights. As long as we believe in each other, we can achieve everything!

Especially during the last months, I was supported a lot by my current team of at the Bernstein Coordination Site. They kept listening to my rants and encouraged me to still go on.

During this thesis, I got to read quite a lot on current climate warming and the hazards ahead of us. Even during our short lifetime, we experience the changes already - not to speak of those living in areas of the world where climate warming does and continually will strike even harder. At the same time, I saw how people, common citizens as well as politicians and those considering themselves to be, either fully deny climate warming or don't want to accept how our far too convenient lifestyle brings us from bad to worse. This is why I want to dearly thank all those who will not stop to stand up and shout out the wake-up calls to the world. I hope the best for us, our children and their children, as well as for this beautiful world we are lucky enough to live in.

Finishing this thesis took quite some time, actually far more than I expected in the beginning or dare to confess in the end. It followed me through different stages of my life from just being a student through to my first steps in a world beyond hard science.

Now I'm happy that after all this very hungry caterpillar is on the edge of becoming a more or less handsome butterfly. Hopefully, this work will help the one or another person with their own project.

The project underlying this thesis was funded by the Deutsche Forschungsgemeinschaft DFG (ME_3575/2-1). 



\section{Declaration}

I hereby declare on oath that I have composed this dissertation by myself and without illegitimate help.

Further, I declare that I have not took an exam for a doctoral degree or have been enrolled for such an exam.

I have already submitted the certificate of fulfilled study requirements to you.

\section{Erklärung}

Hiermit erkläre ich an Eides statt, dass ich die Dissertation selbstständig und ohne unerlaubte Hilfe angefertigt habe.

Weiterhin erkläre ich, dass ich mich noch keiner Promotionsprüfung unterzogen habe oder zu einer solchen Prüfung angemeldet habe.

Der Nachweis der erfolgreichen Ableistung der Studienleistungen liegt Ihnen bereits vor.

Freiburg, August 26, 2019 